\title{
1. NEOGENE PLANKTONIC FORAMINIFER BIOSTRATIGRAPHY, ODP LEG 101, BAHAMAS
}

\author{
Allan J. Melillo²
}

\begin{abstract}
Leg 101 of the Ocean Drilling Program recovered a large volume of Neogene sediments from sites in the Straits of Florida, Little Bahama Bank, and Exuma Sound. In varying amounts, shallow-water, platform-derived carbonate debris is nearly ubiquitous. Reworked planktonic foraminifers are common, especially in the Pliocene-Pleistocene.

At Site 626 in the Straits of Florida, a sequence of Holocene to upper Oligocene sediments was recovered. The greatest Neogene hiatus at this site spans the latest Miocene through Pliocene. Below this, several minor hiatuses are present in a generally conformable sequence.

From the Little Bahama Bank transect (Sites 627, 628, and 630), a nearly complete composite Neogene section was sampled. At Site 627, a major unconformity separates lowermost Miocene sediments from middle to upper Eocene sediments. A second major unconformity occurs at Site 628. Here, middle Miocene sediments lie above uppermost Oligocene deposits.

Sites 632, 633, and 631 in Exuma Sound all bottomed in a thick, lower Pliocene section. The mid-Pliocene is very thin at Sites 633 and 631 , while it is better represented at Site 632 .

Major unconformities at Sites 627 and 628 appear to correlate with periods of elevated sea level, which suggests that carbonate platform shedding may be greatest during this part of the sea-level cycles. One of the salient features of the Bahamas is the lack of any systematic temporal distribution of hiatuses. Only a brief hiatus in the late Pliocene may be regional. It appears that local platform-shedding events were of equal or greater importance in developing the stratigraphy of the Bahamas than regional or eustatic events.
\end{abstract}

\section{INTRODUCTION}

Neogene planktonic foraminifers were recovered at seven sites during Leg 101 in the Bahamas. Three holes drilled in Exuma Sound (Sites 631, 632, and 633) and three drilled off Little Bahama Bank (Sites 627, 628, and 630) formed two transects designed to document the response of carbonate platforms to sealevel changes and the development of carbonate slopes. The oldest sediments reached at Exuma Sound were of early Pliocene age. Site 630 drilling bottomed in lower Pliocene sediments, while drilling at Sites 627 and 628 penetrated the entire Neogene section. At Site 627, an earliest Miocene-Eocene hiatus is present, while at Site 628 a middle Miocene-latest Oligocene hiatus occurs.

Drilling at Site 626, located in the Straits of Florida (Fig. 1), also penetrated the entire Neogene section. Sediment gravity flows in Cores $101-626 \mathrm{C}-13 \mathrm{H}$ to $101-626 \mathrm{C}-18 \mathrm{H}$ were of middle Miocene age, coeval with parts of the Great Abaco Member of the Blake Ridge Formation (Austin, Schlager, et al., 1986).

\section{METHODS}

Samples were prepared by soaking in a sodium carbonate solution, followed by washing on a \#230 U.S. standard sieve (63- $\mu \mathrm{m}$ opening). Relative abundances were estimated of splits of approximately 300 to 500 specimens on a gridded tray. The following abundance categories were used:

$$
\begin{aligned}
& \text { T - Trace, one specimen P } \quad \text { - Present, samples with too few } \\
& \mathrm{R} \text { - Rare, }<3 \% \quad \text { planktonic foraminifers for a } \\
& \text { F - Few, 3-15\% } \\
& \text { C - Common, } 16-30 \% \text { D - Downhole contaminant } \\
& \text { A - Abundant, }>30 \% \quad \text { RW - Reworked }
\end{aligned}
$$

\footnotetext{
${ }^{1}$ Austin, J. A., Jr., Schlager, W. et al., 1988. Proc. ODP, Sci. Results, 101: College Station, TX (Ocean Drilling Program).

2 Department of Geological Sciences, Rutgers University, New Brunswick, NJ 08903. Current address: Chevron U.S.A., Inc., 935 Gravier Street, New Orleans, LA 70112.
}

Preservation was graded using the following scale:

G - Good, little or no overgrowth and/or evidence of dissolution

$\mathrm{M}$ - Moderate, common overgrowth and/or evidence of dissolution

P - Poor, most specimens with overgrowths and/or evidence of dissolution

In general, the preservation of Leg 101 planktonic foraminifers was moderate to poor. In many cases, samples consisted largely of platformderived material. Apparently, the abundant metastable aragonite and high-magnesium calcite present in the platform debris were responsible for the precipitation of calcite overgrowths on many of the planktonic foraminifers. Often these overgrowths were so heavy that identifications could be made only after placing the specimen in an ultrasonic bath for several seconds to dislodge the overgrowths. This was especially com-

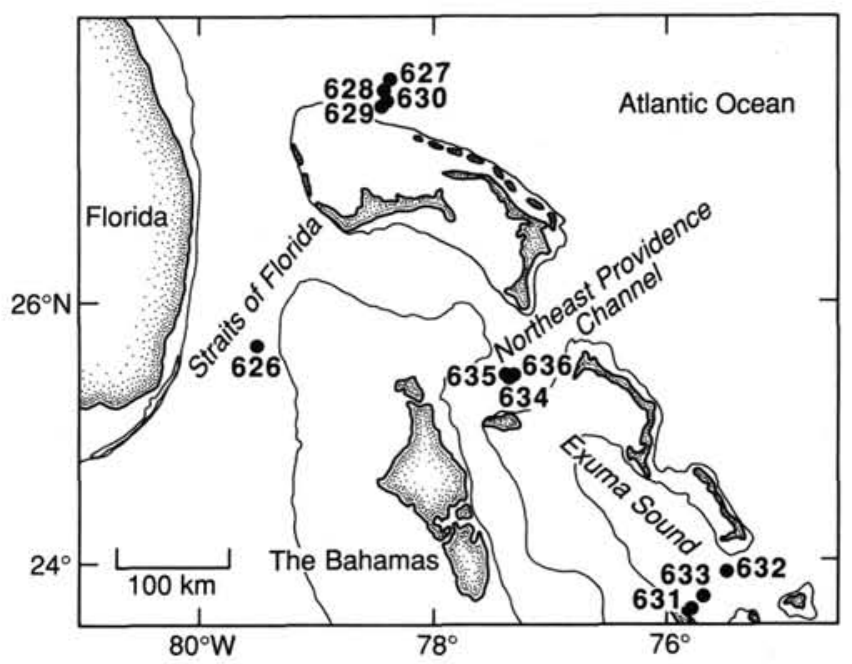

Figure 1. Location map for Leg 101. 
mon at Sites 631, 632, and 633. Fragmentation of tests suggested dissolution in some samples, even though no sites were drilled below the post-Miocene carbonate compensation depth (CCD). It may be that oxidation of the organic matter contained in platform debris locally may raise the carbon dioxide content enough to cause dissolution of tests.

\section{BIOSTRATIGRAPHIC FRAMEWORK}

Taxonomy generally follows that of Bolli and Saunders (1985), although the works of Lamb and Beard (1972), Rogl (1974), Stainforth et al. (1975), Saito et al. (1981), and Kennett and Srinivasan (1983) also were used.

The zonation used here is a slightly modified version of that of Bolli and Saunders (1985) and is shown in Figure 2. Zonation of Bolli and Saunders integrates previous works by Bolli (1957, 1966, and 1970), Bolli and Bermudez (1965), Bolli and PremoliSilva (1973), Cita (1973), and others from warm-water, predominantly Caribbean areas and thus is ideally suited for use in the Bahamas. Only three of the 25 datums used in the MioceneHolocene part of Bolli and Saunders' zonation (1985) were not usable. Two datums, the first occurrence (FO) and the last occurrence (LO) of Globigerinatella insueta Cushman and Stainforth, are difficult to identify consistently. Poor preservation of this thin-walled species may be responsible for the near absence of $G$. insueta throughout its range in the study area. As a result, the boundary between the Catapsydrax dissimilis Zone and the Catapsydrax stainforthi Zone (FO of $G$. insueta) could not be recognized, and these zones were left undifferentiated (Fig. 2). According to Bolli and Saunders (1985), the LO of G. insueta (base Globorotalia fohsi peripheroronda Zone) occurs at or near the FO of Orbulina universa d'Orbigny. Thus, the FO of $O$. universa is used here to mark the base of the Globorotalia fohsi peripheroronda Zone (Fig. 2).

Bolli and Saunders (1985) differentiated the latest Oligocene Globorotalia kugleri Zone from the earliest Miocene Globigerinoides primordius Zone by the first frequent occurrence of $G$. primordius Blow and Banner. During Leg 101, poor preservation made it difficult to identify $G$. primordius consistently. In addition, specimens of $G$. primordius and $G$. sp. cf. G. primordius having two supplementary apertures were observed in the upper Oligocene (Globigerina ciperoensis Zone) at several sites. This supports Lamb and Stainforth's (1976) contention that events in the early evolutionary history of Globigerinoides are unsuitable as zonal criteria. As a result, I combined the two zones into an earliest Miocene Globorotalia kugleri Zone (Fig. 2). Placement of this zone in the Miocene follows Berggren et al. (1985).

Reworking was common and, along with downhole contamination, created problems in determining the age of sediments containing foraminifers diagnostic of adjacent zones. In the presence of reworking, last evolutionary appearances can be of limited value in identifying zonal boundaries. This is because reworking of a taxon into a stratigraphic level above that of its true extinction results in an apparent age that is too old. On the other hand, zonal boundaries based on the first evolutionary appearance of a taxon are not subject to this form of bias, because reworking has no effect on the stratigraphic position of the datum. Therefore, greater importance is attached to first occurrences in identifying zonal boundaries. However, the presence of downhole contamination can result in an age assignment that is too young and must be evaluated carefully.

Ages determined by using planktonic foraminifers are in excellent agreement with ages of the calcareous nannofossils (Watkins and Verbeek, this volume). The few discrepancies are discussed below.

Important species are illustrated with scanning electron micrographs. In most cases, the specimens were cleaned ultrasonically before being photographed so that important morphological features could be seen.

\section{STRAITS OF FLORIDA}

\section{Site 626}

Four holes were drilled at Site 626 in the Straits of Florida in an attempt to reach an inferred, drowned mid-Cretaceous carbonate platform (Austin, Schlager, et al., 1986). Poor hole conditions forced termination of drilling at all four sites; the oldest sediments recovered were of late Oligocene age. Site 626 data were used successfully to document the erosional history of the Gulf Stream for the past $24 \mathrm{~m}$.y. and to document a middle Miocene debris flow at least partly coeval with the Great Abaco Member of the Blake Ridge Formation (Austin, Schlager, et al., 1986; Fulthorpe and Melillo, this volume).

\section{Hole $626 \mathrm{C}$}

Hole $626 \mathrm{C}$ was drilled in $844 \mathrm{~m}$ of water using the extended core barrel (XCB) to a depth of $112.1 \mathrm{mbsf}$ (Cores 101-626C-1X to $101-626 \mathrm{C}-12 \mathrm{X}$ ) and the hydraulic piston corer (HPC) to 179.3 mbsf (Cores 101-626C-13H to 101-626C-19H). A total of $66.3 \mathrm{~m}$ of sediment (37\% recovery) was recovered. Recovery with the $\mathrm{XCB}$ was much poorer $(11 \%)$ than that using the HPC $(79.8 \%)$, and thus fewer data exist for the upper part of the hole than for the lower part.

Determining the age of the sediments in Cores 101-626C-1X through 101-626C-6X was complicated by the extensive reworking of late Miocene and early-middle Pliocene planktonic foraminifers and by the possible downhole caving of Pleistocene forms that characterizes this interval. Specimens of the Pleistocene-Holocene form Globorotalia truncatulinoides truncatulinoides (d'Orbigny) are present in core-catcher samples down through Core 101-626C-6X (Fig. 3). However, Samples 101-626C$4 \mathrm{X}-1,25-27 \mathrm{~cm}$, and 101-626C-5X-1, 25-27 cm, do not contain this species, and only a single specimen is present in Sample 101-626C-6X-1, 25-27 cm (Fig. 3). It may be that the specimens of $G$. truncatulinoides truncatulinoides in Cores 101-626C-4X through 101-626C-6X are caved and that this interval contains pre-Pleistocene sediments. In that case, the most likely age of the sediments would be late Miocene to early Pliocene, based on the presence of a large number of species characteristic of this interval (Fig. 3; Bolli and Saunders, 1985). However, most of these species are also common as reworked fossils above Core 101-626C-4X, where specimens of $G$. truncatulinoides truncatulinoides occur consistently and are more abundant (Fig. 3). It seems likely that the absence of $G$. truncatulinoides truncatulinoides is caused by dilution of the in-situ fauna with the much more abundant reworked forms.

If considered in-situ, the presence of Globorotalia ungulata Bermudez in Sample 101-626C-5X-1, 25-27 cm, indicates a Holocene age (Globorotalia fimbriata Zone) for the sediments down to this depth (Fig. 3). This is supported by the observed calcareous nannofossils, which also indicate a Holocene age (NN21) down to this depth (Watkins and Verbeek, this volume). Below this depth, in Sections 101-626C-5X, CC through 101-626C-6X, $\mathrm{CC}$, the absence of any Holocene marker species, along with the presence of $G$. truncatulinoides truncatulinoides, indicates a Pleistocene age, which also agrees with the calcareous nannofossil data of Watkins and Verbeek (this volume).

Because of poor recovery in Core 101-626C-7X, only the corecatcher sample was examined. The fine-grained volcanic ash contains few foraminifers, mostly in the less than $250-\mu \mathrm{m}$ fraction. The absence of any Pliocene marker species and the presence of Globorotalia plesiotumida Blow and Banner, G. pseudomiocenica Bolli and Bermudez, and Globigerinoides extremus Bolli indicate a latest Miocene age (upper Neogloboquadrina humerosa Zone) for these sediments (Fig. 3). This suggests that the specimens of Globorotalia margaritae margaritae Bolli and Bermudez in Section 101-626B-2W, CC at 80.3 mbsf are caved from the washed interval above. 
NEOGENE PLANKTONIC FORAMINIFER BIOSTRATIGRAPHY

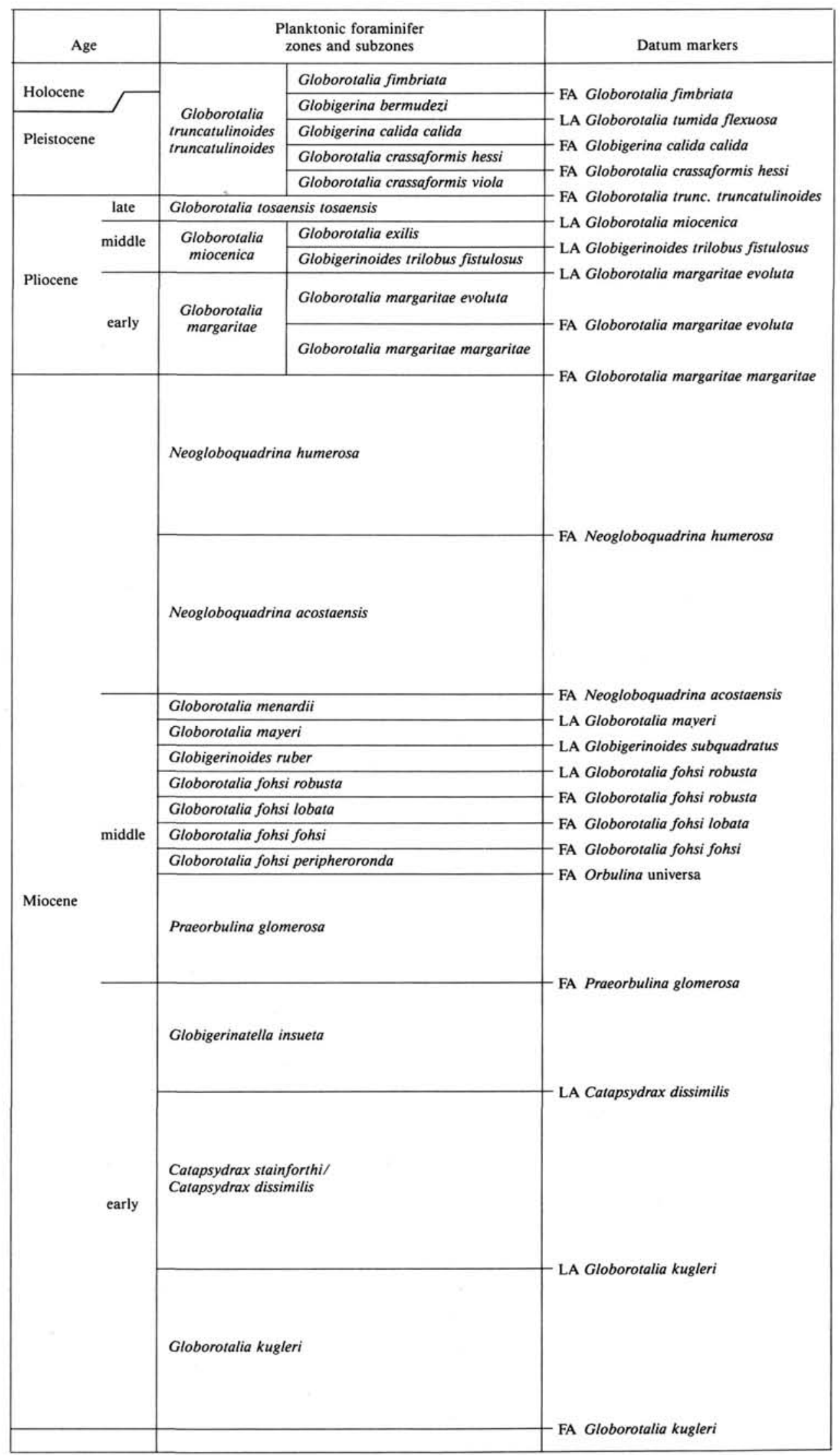

Figure 2. Planktonic foraminifer zonation, modified from Bolli and Saunders (1985). FA = first appearance; $\mathbf{L A}=$ last appearance. 


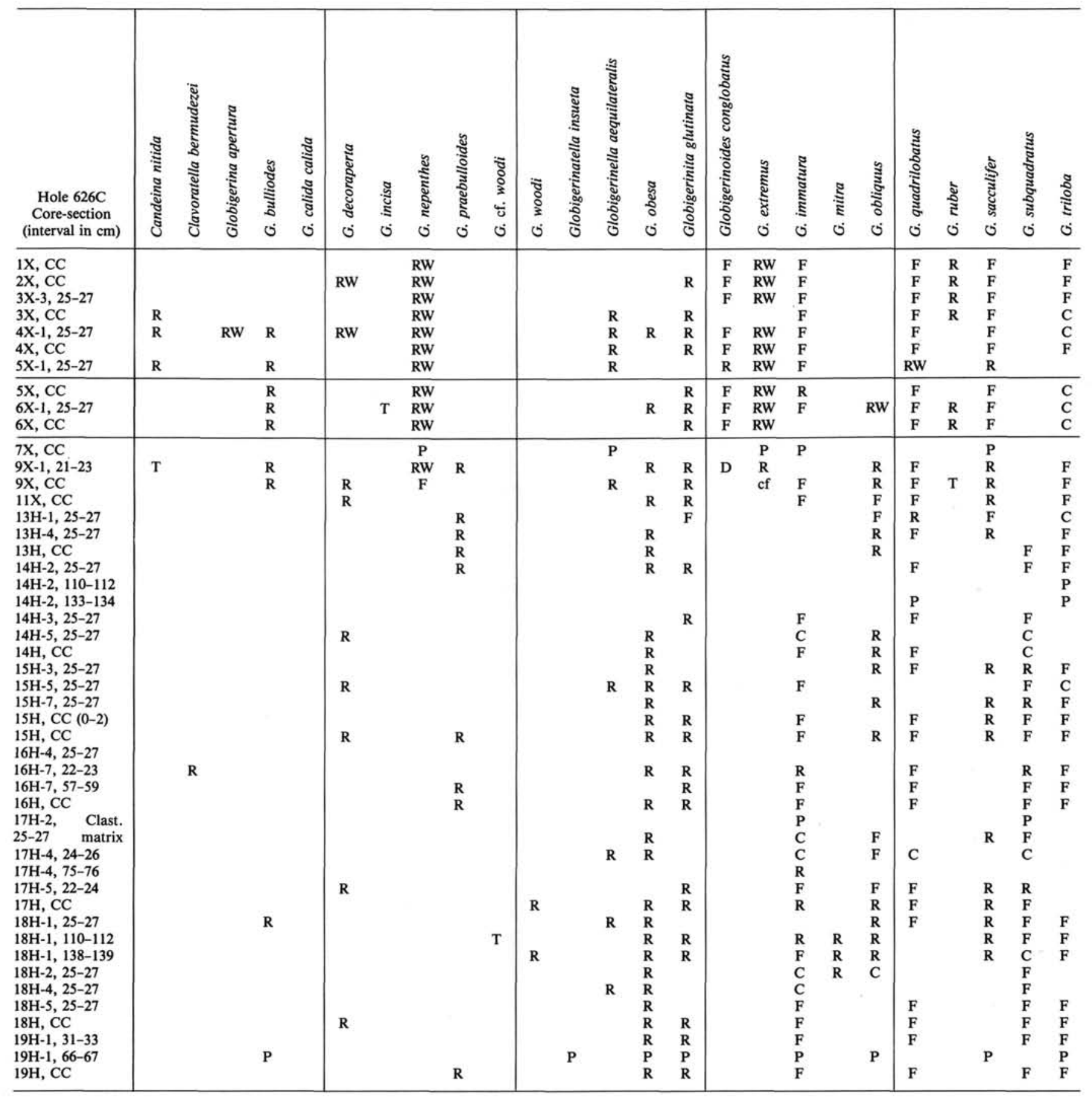

Figure 3. Stratigraphic ranges of selected planktonic foraminifers at Hole 626C.

Core 101-626C-8X was empty when retrieved. Sample 101$626 \mathrm{C}-9 \mathrm{X}-1,21-23 \mathrm{~cm}$, contains morphologically primitive specimens of Neogloboquadrina humerosa (Takayanagi and Saito) as well as Globorotalia plesiotumida, Globigerinoides extremus, Candeina nitida d'Orbigny, and Sphaeroidinellopsis paenedehiscens Blow ( $=S$. sphaeroides Lamb sensu Bolli and Saunders, 1985), which indicates the presence of the latest Miocene Neogloboquadrina humerosa Zone (Fig. 3).

Section 101-626C-9X, CC contains N. acostaensis (Blow) without $N$. humerosa, identifying the late Miocene Neogloboquadrina acostaensis Zone (Fig. 3). The planktonic foraminifer assemblage also contains forms that are transitional between Globigerinoides obliquus Bolli and $G$. extremus but that lack the characteristic latest Miocene species found in Sample 101626C-9X-1, 21-23 cm (Fig. 3).

Core 101-626C-10X was empty when retrieved. Poor recovery in Core 101-626C-11X allowed examination of only the corecatcher sample. In that sample, the presence of Globorotalia mayeri Cushman and Ellisor without Globigerinoides subquadratus Bronnimann of Cordey, 1967 ( = G. ruber sensu Bolli and Saunders, 1982, 1985) indicates the presence of the late middle Miocene Globorotalia mayeri (= siakensis) Zone (Fig. 3). Because of poor recovery in the interval penetrated by Cores 101$626 \mathrm{C}-9 \mathrm{X}$ to $101-626 \mathrm{C}-11 \mathrm{X}$, I was unable to determine the exact position and nature of the middle/late Miocene boundary (Fig. 3). 


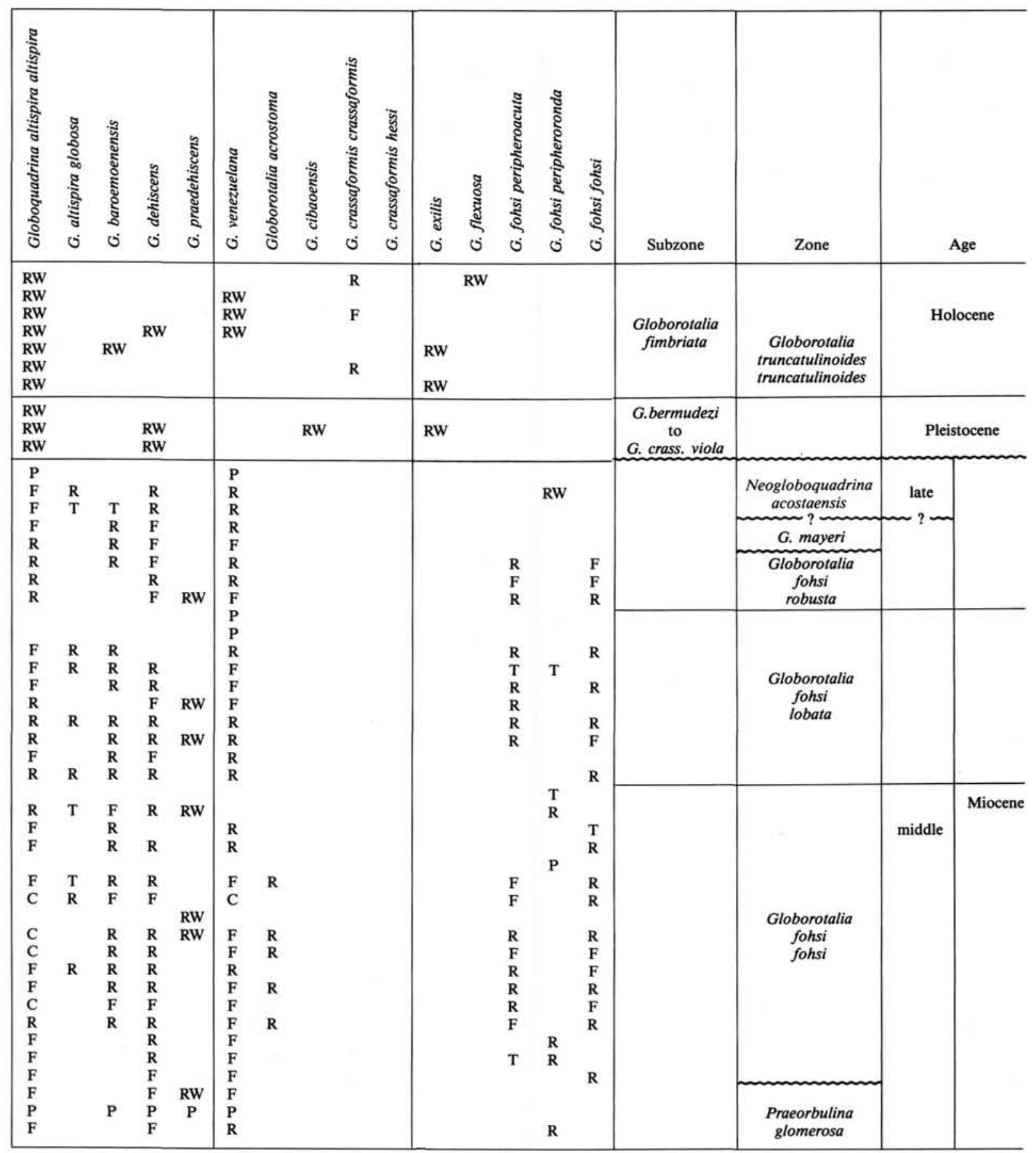

Figure 3 (continued).

Core 101-626C-12X was empty when retrieved. The sediments in Cores $101-626 \mathrm{C}-13 \mathrm{H}$ to $101-626 \mathrm{C}-18 \mathrm{H}$ were deposited predominantly by carbonate sediment gravity flows. These sediments are characterized by abundant shallow-water-platform benthic foraminifers and other debris and by planktonic foraminifers that generally display some degree of overgrowths. This was typical of the sediments containing platform debris recovered at all sites during Leg 101. Reworking of planktonic foraminifers was not unusual but generally was from only slightly older sediments.
The sediments in Sample 101-626C-13H-1, 25-27 cm, also correlate with the Globorotalia mayeri Zone, based on the presence of the nominate taxon without Globigerinoides subquadratus (Fig. 3). This sample also contains apparently reworked specimens of Globorotalia fohsi lobata Bermudez (Fig. 3). Reworking is suggested by the uppermost occurrence in Sample 101-626C$13 \mathrm{H}-4,25-27 \mathrm{~cm}$, of Globorotalia fohsi robusta Bolli and $G$. praemenardii Cushman and Stainforth, species that Bolli and Saunders (1985) showed ranging to the top of the Globorotalia fohsi robusta Zone, above the extinction of $G$. fohsi lobata (Fig. 


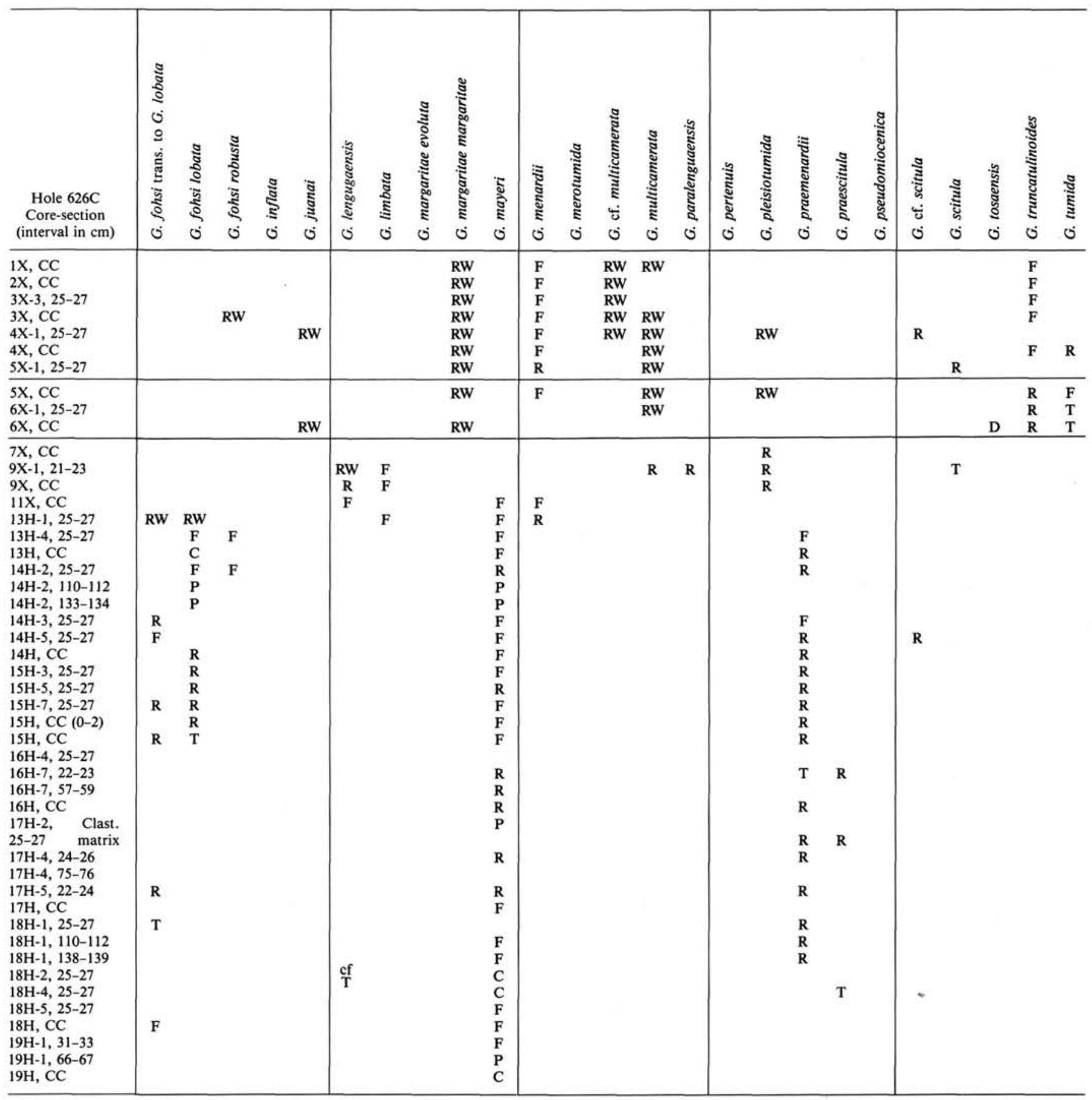

Figure 3 (continued).

3). Based on the presence of these species, the sediments in this sample correlate with the Globorotalia fohsi robusta Zone (Fig. $3)$. This zone is present in sediments down to the level of Sample $101-626 \mathrm{C}-14 \mathrm{H}-2,25-27 \mathrm{~cm}$, where the nominate taxon first occurs (Fig. 3).

The top of the Globorotalia fohsi lobata Zone was placed in Sample 101-626C-14H-2, 133-134 cm, where the nominate taxon occurs without $G$. fohsi robusta (Fig. 3). The FO of $G$. fohsi lobata in Section 101-626C-15H, CC marks the base of the zone (Fig. 3).

The presence of $G$. fohsi fohsi Cushman and Ellisor, without G. fohsi lobata in Samples 101-626C-16H-4, 25-27 cm, through
101-626C-18H, CC, indicates the presence of the Globorotalia fohsi fohsi Zone (Fig. 3). Several clasts from Cores 101-626C$16 \mathrm{H}$ and $101-626 \mathrm{C}-17 \mathrm{H}$, obtained from C. Fulthorpe, were examined to determine the degree of reworking in the debris flows that characterize the sediments of Cores 101-626C-13H through $101-626 \mathrm{C}-18 \mathrm{H}$. Two of the clasts, Samples 101-626C-16H-4, 25$27 \mathrm{~cm}$, and $101-626 \mathrm{C}-17 \mathrm{H}-4,75-76 \mathrm{~cm}$, contain specimens of Orbulina universa d'Orbigny, which indicates a middle Miocene age (Fig. 3). Sample 101-626C-16H-7, 57-59 cm, contains Globorotalia fohsi fohsi without the younger members of the $G$. fohsi group and thus may be in the same planktonic foraminifer zone as the enclosing sediments (Fig. 3). A middle Miocene age 


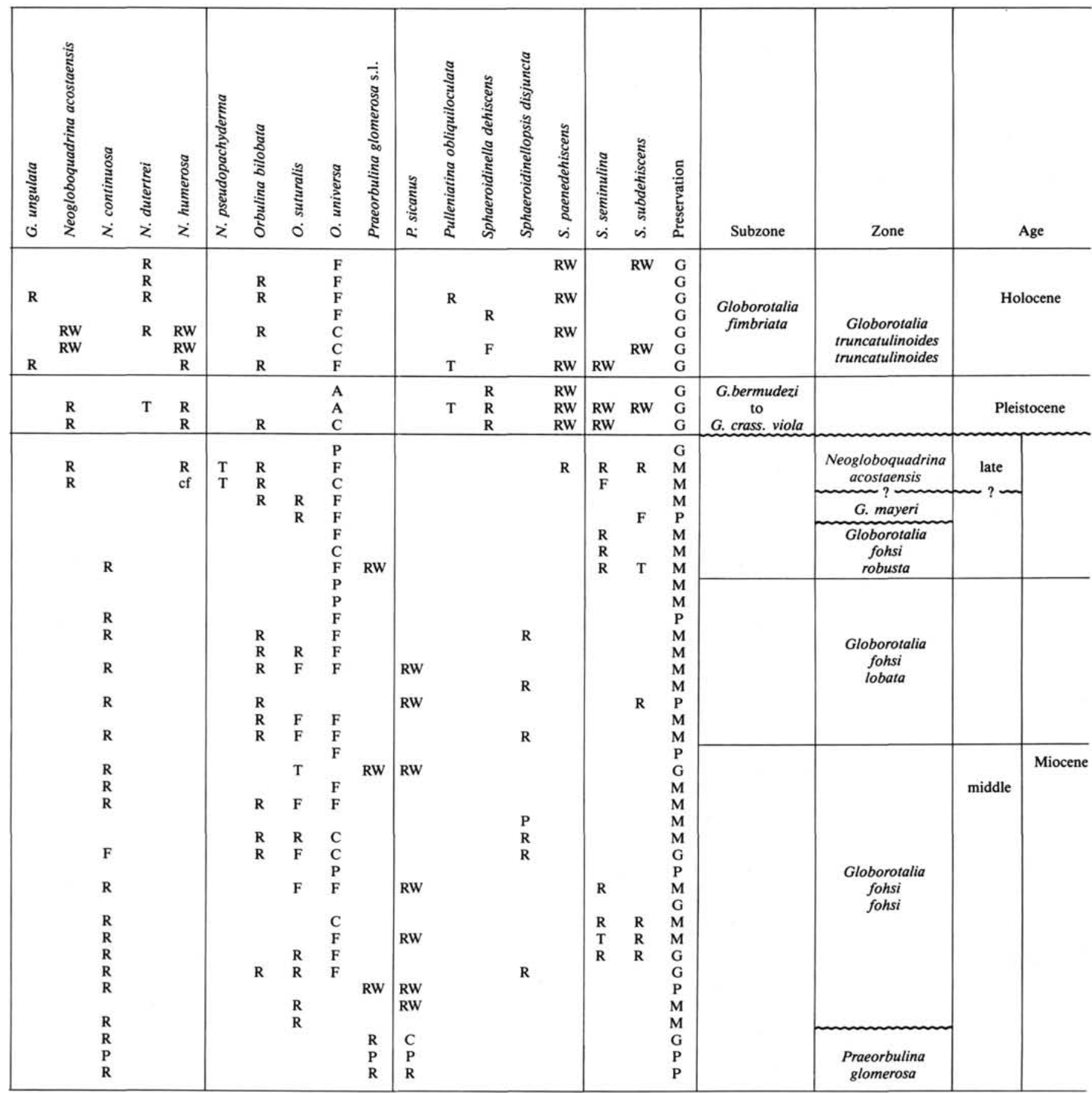

Figure 3 (continued).

was indicated for Sample 101-626C-17H-2, 25-27 cm, based on the presence of Sphaeroidinellopsis disjuncta (Finlay) (Fig. 3). Thus most of the material composing the debris flows was not derived from markedly older sediments, and some is of the age of the episodes of slope failure.

An erosional(?) unconformity is present between Cores 101$626 \mathrm{C}-18 \mathrm{H}$ and $101-626 \mathrm{C}-19 \mathrm{H}$, coinciding with the base of the debris flows (Fig. 3; Austin, Schlager, et al., 1986; Fulthorpe and Melillo, this volume). The presence of Praeorbulina glomerosa (Blow) without Orbulina universa or $O$. suturalis Bronnimann in Sample 101-626C-19H-1, 31-33 cm, indicates that these sediments occur in the earliest middle Miocene Praeorbulina glomerosa Zone (Fig. 3). Thus, the early middle Miocene
Globorotalia fohsi peripheroronda Zone is missing at Site 626 (Fig. 3).

\section{Hole $626 D$}

Hole $626 \mathrm{D}$ was washed to $179.4 \mathrm{mbsf}$, then rotary cored to $446.8 \mathrm{mbsf}$. Twenty-eight cores were recovered having $9.39 \mathrm{~m}$ of sediments (3.3\% recovery) before the core barrel and drill string became stuck and the hole was abandoned (Austin, Schlager, et al., 1986).

Sediments in Cores 101-626D-1R, 101-626D-3R, and 101626D-4R can be correlated with the Praeorbulina glomerosa Zone, based on the presence of the nominate taxon (Fig. 4). Section 101-626D-1R, CC is composed mainly of younger mid- 


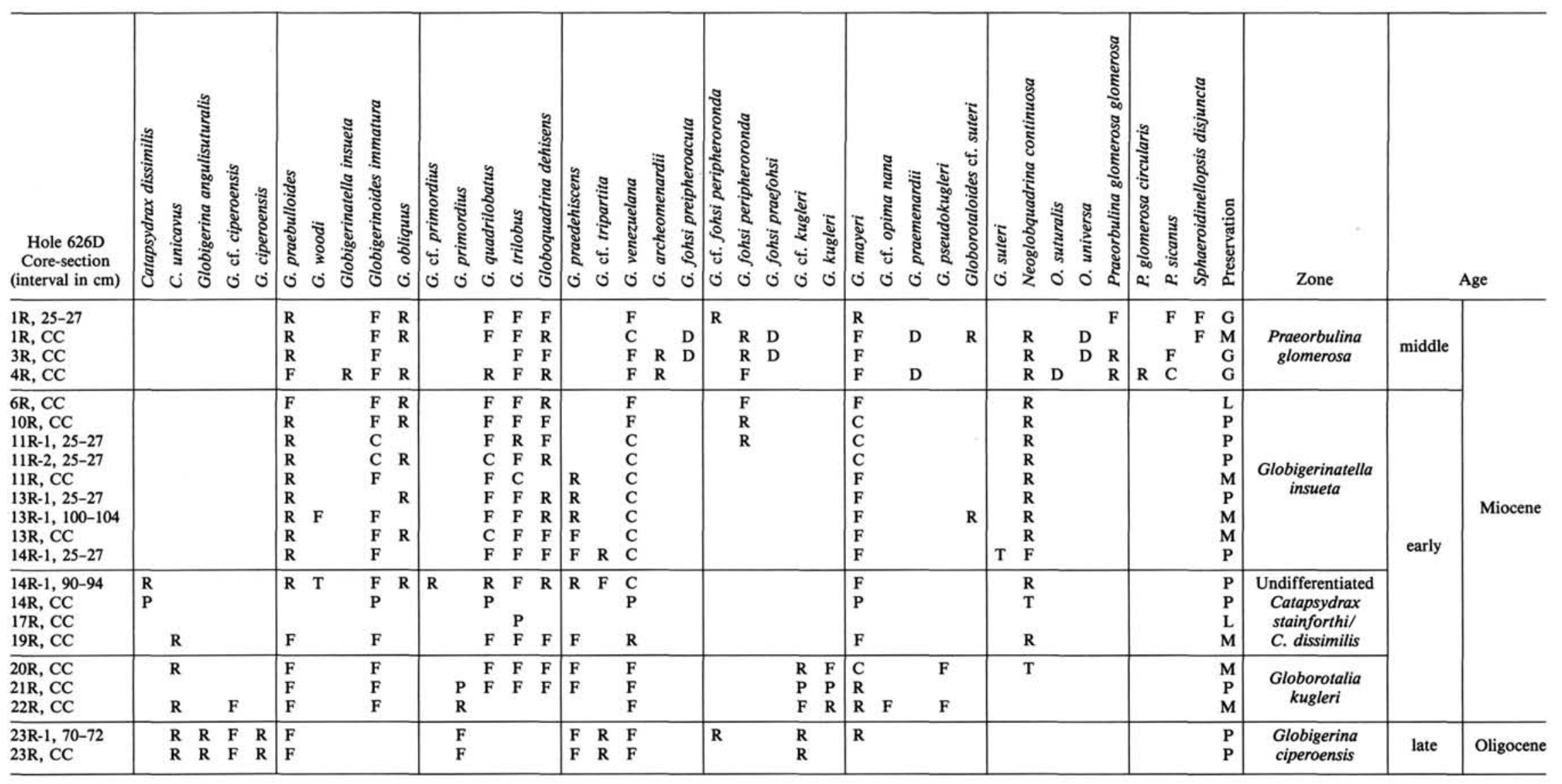

Figure 4. Stratigraphic ranges of selected planktonic foraminifers at Hole 626D. 
dle Miocene sediments, which are considered downhole contaminants (Fig. 4). Cores 101-626D-2R and 5R were empty when retrieved.

The interval between Section 101-626D-6R, CC and Sample 101-626D-14R-1, 25-27 cm, correlates with the early Miocene Globigerinatella insueta Zone (Fig. 4). This is based on the absence of Praeorbulina glomerosa s.l. and Catapsydrax dissimilis (Cushman and Bermudez) and C. unicavus Bolli, Loeblich, and Tappan (Fig. 4). No specimens of Globigerinatella insueta Cushman and Stainforth were present at Site 626 and, in fact, were rarely seen at any of the Leg 101 sites that penetrated this stratigraphic interval. This probably results from the general paucity of this species and the poor preservation from dissolution and/ or overgrowths that often characterize this interval. Thus, the absence of $G$. insueta in Cores 101-626D-6R to 101-626D-14R does not negate the zonal assignments. Owing to the absence of recovery in Core 101-626D-5R and the very poor recovery in general, no definitive statement regarding the nature of the lower/middle Miocene boundary at Site 626 can be made. However, it may be that this boundary is conformable or marked by a minor hiatus below the resolution of the planktonic foraminifer zonation.

Sediments of the undifferentiated Catapsydrax stainforthi and C. dissimilis Zones were identified in the interval between Samples 101-626D-14R-1, 90-94 cm (where $C$. dissimilis last occurs) and 101-626D-19R, CC (Fig. 4).

The LO of Globorotalia kugleri Bolli in Section 101-626D20R, CC marks the top of the earliest Miocene Globorotalia kugleri Zone (Fig. 4). Core 101-626D-21R was empty when retrieved. The base of the zone (and the base of the Miocene as used here) was placed at the level of Section 101-626D-22R, CC, where G. kugleri first occurs (Fig. 4).

The sediments in Sample 101-626D-23R-1, 70-74 cm, contain Globigerina ciperoensis ciperoensis Bolli and thus indicate the presence of the uppermost Oligocene Globigerina ciperoensis Zone (Fig. 4). The absence of any demonstrable hiatus suggests that the Oligocene/Miocene boundary section at Site 626 is complete (Fig. 4).

\section{LITTLE BAHAMA BANK TRANSECT-SITES 627, 628, AND 630}

Sites 627,628 , and 630 sampled the northern slope of Little Bahama Bank (LBB) to compare the "accretionary" slope there with the "bypass" slope that characterizes Exuma Sound (Austin, Schlager, et al., 1986). Site 629 was not successfully spudded and is not discussed here.

\section{Site 627}

Site 627 was drilled for two reasons: (1) to sample an inferred buried Cretaceous shallow-water platform and (2) to serve as the seaward end of the LBB transect (Austin, Schlager, et al., 1986). Two holes were drilled in approximately $1028 \mathrm{~m}$ of water: Hole 627A, where the first core did not recover the sediment surface and was abandoned; and Hole $627 \mathrm{~B}$, which penetrated to 536 mbsf. Sixty cores were retrieved, recovering $344.2 \mathrm{~m}$ of sediment $(64 \%$ recovery). Neogene sediments were observed down to 181.4 mbsf (Section 101-627B-19X, CC). Recovery in this part of the section was $91 \%$ (163.92 m).

\section{Hole 627B}

Sediments that correlate with the Holocene Globorotalia fimbriata Zone were observed down to the level of Sample 101$627 \mathrm{~B}-1 \mathrm{H}-4,25-27 \mathrm{~cm}$, based on the presence of $G$. ungulata (Fig. 5). Also present in this interval are rare, reworked specimens of the Pliocene species G. miocenica Palmer, G. exilis Blow, and G. pertenuis Beard, as well as the lower Pleistocene form G. crassaformis hessi Bolli and Premoli-Silva (Fig. 5). The reworked foraminifers are more poorly preserved than the insitu fauna and usually exhibit a yellowish color when wet.

A Pleistocene age (Globorotalia truncatulinoides truncatulinoides Zone) was assigned to the sediments in Section 101-627B$1 \mathrm{H}, \mathrm{CC}$, based on the absence of any Holocene marker species and the presence of the nominate taxon (Fig. 5). The presence of apparently in-situ specimens of $G$. crassaformis viola Blow suggests a correlation with the earliest Pleistocene (Globorotalia crassaformis viola Subzone) (Fig. 5). Also present are forms that are transitional between $G$. crassaformis ronda Blow and $G$. crassaformis hessi (referred to G. sp. cf. G. crassaformis hessi), which suggests a Pleistocene level below the FO of $G$. crassaformis hessi (i.e., the Globorotalia crassaformis viola Subzone) (Figs. 2, 5).

Core 101-627B-1H was recovered with a gap of approximately $3.7 \mathrm{~m}$ between the lowest sediments in the core barrel and the core catcher (Austin, Schlager, et al., 1986). According to ODP convention, the core-catcher sample is placed below the last section recovered and treated as a separate section. In this case, only $1.05 \mathrm{~m}$ separated the Holocene sediments of Sample 101627B-1H-4, 25-27 cm, from the lowermost Pleistocene sediments in the core-catcher sample (Fig. 5; Austin, Schlager, et al., 1986). This suggests the presence of an unconformity between these two samples, which may be an artifact. However, if recovery were complete, less than $5 \mathrm{~m}$ should have separated these sediments, which again suggests the presence of an unconformity. I believe that a Holocene/Pleistocene unconformity is present at Site 627, with sediments of the Globigerina bermudezi Subzone through Globorotalia crassaformis hessi Subzone missing (Figs. 2 and 5).

An unconformity appears to separate lower Pleistocene sediments from middle Pliocene sediments (Fig. 5). Sample 101627B-2H-1, 25-27 cm, contains G. miocenica and $G$. exilis, which indicates the presence of the Globorotalia miocenica Zone (Fig. 5). The absence of Globigerinoides trilobus fistulosus Schubert in this sample indicates the upper part of the zone; that is, the Globorotalia exilis Subzone (Fig. 5). This subzone was assigned to sediments extending down to the level of Sample 101-627B$3 \mathrm{H}-5,25-27 \mathrm{~cm}$ (Fig. 5). Samples 101-627B-3H-6, 25-27 cm, and 101-627B-3H, CC correlate with the Globigerinoides trilobus fistulosus Subzone, based on the presence of the nominate taxon (Fig. 5). The presence of Globorotalia multicamerata Cushman and Jarvis in the core-catcher sample indicates the lower part of the subzone (Fig. 5; Bolli and Saunders, 1985).

Lower Pliocene sediments that correlate with the Globorotalia margaritae Zone are first encountered in Sample 101-627B$4 \mathrm{H}-2,50-52 \mathrm{~cm}$, based on the presence of $G$. margaritae margaritae (Fig. 5). The presence of $G$. exilis in this sample suggests a correlation with the uppermost Globorotalia margaritae evoluta Subzone (Fig. 5). Sediments of this subzone extend to the level of Section 101-627B-7H, CC, where both $G$. margaritae margaritae and G. margaritae evoluta Cita first occur (Fig. 5).

An unconformity appears to separate upper Miocene sediments from lower Pliocene sediments at Site 627 (Fig. 5). The absence of G. margaritae s.1. from Sample 101-627B-8H-1, 25$27 \mathrm{~cm}$, and the presence of G. plesiotumida and Candeina nitida suggest the latest Miocene upper Neogloboquadrina humerosa Zone (Fig. 5; Bolli and Saunders, 1985). It appears that sediments of the lower Pliocene Globorotalia margaritae margaritae Subzone are missing (Fig. 5). Uppermost Miocene sediments of the Neogloboquadrina humerosa Zone extend downward to Sample 101-627B-10H-1, 25-27 cm, where Globorotalia plesiotumida first occurs (Fig. 5).

The sediments in Core 101-627B-11H and most of Core 101$627 \mathrm{~B}-12 \mathrm{H}$ are part of a slump deposit. Middle Miocene planktonic foraminifers are present in this unit, and can be used to identify repeated sections within the slump (Fig. 5). 


\begin{tabular}{|c|c|c|c|c|c|c|c|c|c|c|c|c|c|c|c|c|c|c|c|c|c|c|c|c|c|}
\hline $\begin{array}{c}\text { Hole 627B } \\
\text { Core-section } \\
\text { (interval in cm) }\end{array}$ & 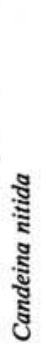 & 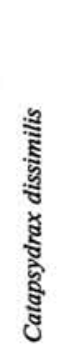 & 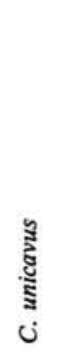 & 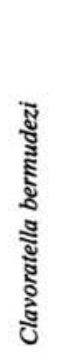 & 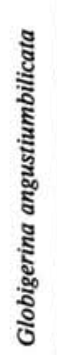 & 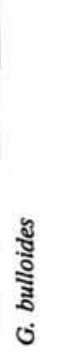 & 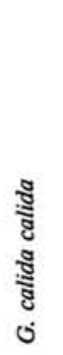 & 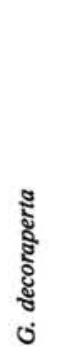 & $\begin{array}{l}5 \\
\frac{5}{0} \\
0\end{array}$ & : & 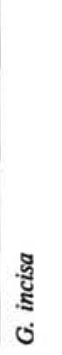 & 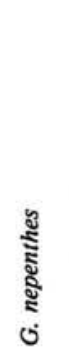 & 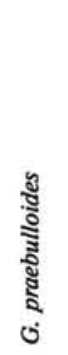 & $\begin{array}{l}\text { हैँ } \\
\text { हैँ } \\
\text { है }\end{array}$ & $\begin{array}{c}\tilde{\Xi} \\
\vdots \\
\vdots \\
ن\end{array}$ & 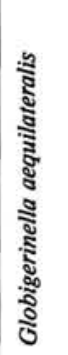 & $\begin{array}{l}\tilde{g} \\
\delta \\
0\end{array}$ & 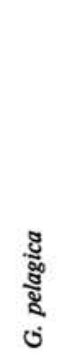 & 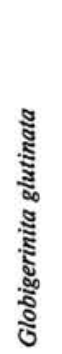 & 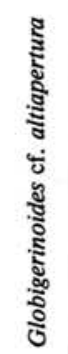 & $\begin{array}{l}\vdots \\
\vdots \\
0 \\
\dot{0} \\
0 \\
0\end{array}$ & 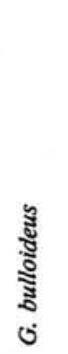 & 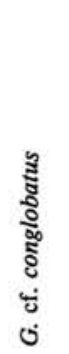 & 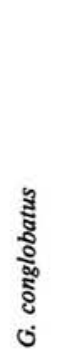 & 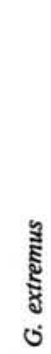 \\
\hline $\begin{array}{l}1 \mathrm{H}-1,25-27 \\
1 \mathrm{H}-2,25-27 \\
1 \mathrm{H}-4,25-27\end{array}$ & $\begin{array}{l}\mathrm{T} \\
\mathbf{R}\end{array}$ & & & & & & $\begin{array}{l}\mathbf{R} \\
\mathbf{R} \\
\mathrm{R}\end{array}$ & & & $\mathbf{R}$ & & & & & & $\begin{array}{l}\mathrm{R} \\
\mathrm{F}\end{array}$ & & $\mathbf{R}$ & $\begin{array}{l}\mathbf{R} \\
\mathbf{R} \\
\mathrm{R}\end{array}$ & & & & & $\begin{array}{l}\mathbf{R} \\
\mathbf{R}\end{array}$ & \\
\hline $\begin{array}{l}1 \mathrm{H}, \mathrm{CC} \\
2 \mathrm{H}-3,25-27 \\
2 \mathrm{H}-6,25-27 \\
2 \mathrm{H}, \mathrm{CC}\end{array}$ & & & & & & & & $\mathbf{R}$ & & $\mathbf{R}$ & & & & & & $\begin{array}{l}\mathrm{F} \\
\mathrm{R} \\
\mathrm{F} \\
\mathrm{R}\end{array}$ & & R & $\begin{array}{l}\mathrm{R} \\
\mathrm{R} \\
\mathrm{R} \\
\mathrm{R}\end{array}$ & & & & & $\begin{array}{l}\mathrm{R} \\
\mathrm{R} \\
\mathrm{R} \\
\mathrm{R}\end{array}$ & \\
\hline $\begin{array}{l}3 \mathrm{H}-3,25-27 \\
3 \mathrm{H}-5,25-27\end{array}$ & $\mathrm{~T}$ & & & & & R & & & & & & & & & & $\begin{array}{l}\mathbf{F} \\
\mathbf{R}\end{array}$ & $\mathbf{R}$ & & $\begin{array}{l}\mathbf{R} \\
\mathrm{R}\end{array}$ & & & & & $\begin{array}{l}\mathrm{R} \\
\mathrm{R}\end{array}$ & $\mathbf{R}$ \\
\hline $\begin{array}{l}3 \mathrm{H}-6,25-27 \\
3 \mathrm{H}, \mathrm{CC}\end{array}$ & & & & & & & & $\mathbf{R}$ & & $\begin{array}{l}\mathbf{R} \\
\mathbf{R}\end{array}$ & R & & & $\begin{array}{l}\mathbf{R} \\
\mathbf{R}\end{array}$ & & $\begin{array}{l}\mathrm{F} \\
\mathrm{F}\end{array}$ & $\begin{array}{l}\mathbf{R} \\
\mathbf{R}\end{array}$ & & $\begin{array}{l}\mathbf{R} \\
\mathrm{R}\end{array}$ & & & & & $\begin{array}{l}\mathrm{R} \\
\mathrm{R}\end{array}$ & $\mathbf{R}$ \\
\hline $\begin{array}{l}4 \mathrm{H}-2,50-52 \\
4 \mathrm{H}, \mathrm{CC} \\
5 \mathrm{H}, \mathrm{CC} \\
6 \mathrm{H}, \mathrm{CC} \\
7 \mathrm{H}-2,25-27 \\
7 \mathrm{H}, \mathrm{CC}\end{array}$ & $\begin{array}{l}\mathrm{T} \\
\mathrm{R} \\
\mathrm{R} \\
\mathrm{R} \\
\mathrm{T}\end{array}$ & & & F & & $\begin{array}{l}\mathbf{R} \\
\mathbf{R}\end{array}$ & & $\begin{array}{l}\mathrm{R} \\
\mathrm{R} \\
\mathrm{R} \\
\mathrm{R} \\
\mathrm{R}\end{array}$ & & $\mathbf{R}$ & & $\begin{array}{l}\mathbf{F} \\
\mathbf{R}\end{array}$ & & R & & $\begin{array}{l}\mathrm{R} \\
\mathrm{R} \\
\mathrm{R}\end{array}$ & $\begin{array}{l}\mathrm{R} \\
\mathbf{R} \\
\mathbf{R} \\
\mathbf{R}\end{array}$ & & $\begin{array}{l}\mathbf{R} \\
\mathbf{R} \\
\mathbf{R} \\
\mathrm{R} \\
\mathrm{R} \\
\mathrm{R}\end{array}$ & & & & $\mathbf{R}$ & $\begin{array}{l}\mathrm{R} \\
\mathrm{R} \\
\mathrm{F} \\
\mathrm{F} \\
\mathrm{F} \\
\mathrm{R}\end{array}$ & $\begin{array}{l}R \\
\text { R } \\
\text { F } \\
\text { C } \\
\text { F } \\
\text { F }\end{array}$ \\
\hline $\begin{array}{l}8 \mathrm{H}-1,25-27 \\
8 \mathrm{H}-2,25-27 \\
8 \mathrm{H}-3,25-27 \\
8 \mathrm{H}-4,25-27 \\
8 \mathrm{H}-6,25-27 \\
8 \mathrm{H}, \mathrm{CC} \\
9 \mathrm{H}-2,25-27 \\
9 \mathrm{H}-3,25-27 \\
9 \mathrm{H}-4,25-27 \\
9 \mathrm{H}-6,25-27 \\
9 \mathrm{H}, \mathrm{CC} \\
10 \mathrm{H}-1,25-27 \\
10 \mathrm{H}-2,25-27 \\
10 \mathrm{H}-4,25-27 \\
10 \mathrm{H}-5,25-27 \\
10 \mathrm{H}, \mathrm{CC} \\
11 \mathrm{H}-1,25-27 \\
11 \mathrm{H}-2,25-27 \\
11 \mathrm{H}-3,25-27 \\
11 \mathrm{H}-4,25-27 \\
11 \mathrm{H}-5,25-27 \\
11 \mathrm{H}-6,25-27 \\
11 \mathrm{H}, \mathrm{CC} \\
12 \mathrm{H}-5,25-27 \\
12 \mathrm{H}-6,25-27 \\
12 \mathrm{H}-7,25-27 \\
12 \mathrm{H}, \mathrm{CC} \\
13 \mathrm{H}-1,25-27 \\
13 \mathrm{H}-3,25-27 \\
13 \mathrm{H}-4,25-27 \\
13 \mathrm{H}-5,25-27 \\
13 \mathrm{H}, \mathrm{CC} \\
14 \mathrm{H}-2,25-27 \\
14 \mathrm{H}-5,25-27 \\
14 \mathrm{H}, \mathrm{CC} \\
15 \mathrm{H}-2,28-30 \\
15 \mathrm{H}-5,25-27 \\
15 \mathrm{H}, \mathrm{C} \mathrm{C} \\
16 \mathrm{H}, \mathrm{CC} \\
17 \mathrm{X}-1,25-27 \\
17 \mathrm{X}-3,25-27 \\
17 \mathrm{X}, \mathrm{CC} \\
18 \mathrm{X}, \mathrm{CC} \\
19 \mathrm{X}-1,60-62 \\
19 \mathrm{X}, \mathrm{CC}\end{array}$ & $\begin{array}{l}\mathrm{R} \\
\mathrm{T} \\
\\
\mathrm{T} \\
\mathrm{R}\end{array}$ & RW & $\mathbf{R}$ & $T$ & $\begin{array}{l}\mathbf{R} \\
\mathbf{R}\end{array}$ & $\begin{array}{l}\mathrm{F} \\
\mathrm{R} \\
\mathrm{R} \\
\\
\\
\mathrm{T}\end{array}$ & & $\begin{array}{l}\mathrm{R} \\
\mathrm{T} \\
\\
\mathrm{R} \\
\\
\mathrm{R} \\
\mathrm{R} \\
\mathrm{R} \\
\mathrm{R} \\
\mathrm{R} \\
\mathrm{R} \\
\mathrm{F} \\
\mathrm{R} \\
\mathrm{F} \\
\mathrm{F} \\
\\
\mathrm{R} \\
\mathrm{R} \\
\mathrm{R} \\
\mathrm{F} \\
\mathrm{R} \\
\\
\mathrm{R} \\
\mathrm{R} \\
\mathrm{R} \\
\mathrm{R} \\
\\
\mathrm{F} \\
\mathrm{R} \\
\mathrm{R} \\
\mathrm{R} \\
\mathrm{R} \\
\mathrm{R} \\
\mathrm{R}\end{array}$ & 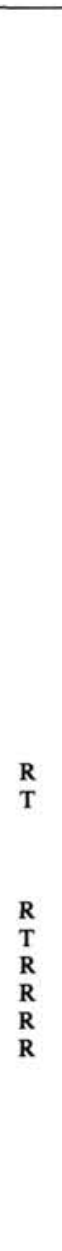 & 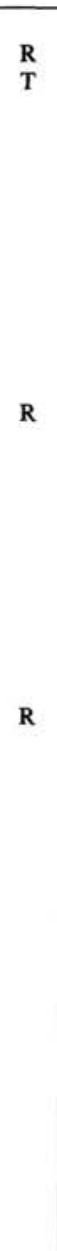 & & $\begin{array}{l}\text { F } \\
\text { R } \\
\text { R } \\
\text { R } \\
\text { T } \\
\text { F } \\
\text { R } \\
\text { F } \\
\text { F } \\
\text { F } \\
\text { F } \\
\text { F } \\
\text { F } \\
\text { R } \\
\text { R } \\
\text { R } \\
\text { T } \\
\end{array}$ & $\begin{array}{l}\mathrm{R} \\
\mathrm{T} \\
\mathrm{T} \\
\mathrm{R} \\
\mathrm{R} \\
\mathrm{R} \\
\mathrm{R} \\
\mathrm{R} \\
\mathrm{R}\end{array}$ & & $\mathbf{T}$ & $\begin{array}{l}\mathrm{R} \\
\mathrm{F} \\
\mathrm{F} \\
\mathrm{F} \\
\mathrm{F} \\
\mathrm{R} \\
\mathrm{R} \\
\mathrm{F} \\
\\
\mathrm{R} \\
\mathrm{R} \\
\mathrm{R} \\
\mathrm{R} \\
\mathrm{F} \\
\mathrm{R} \\
\mathrm{R}\end{array}$ & 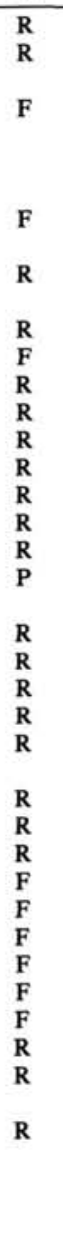 & $\begin{array}{l}\mathbf{R} \\
\mathbf{R} \\
\mathbf{R}\end{array}$ & $\begin{array}{l}\mathrm{R} \\
\mathrm{R} \\
\\
\\
\mathrm{R} \\
\mathrm{R} \\
\mathrm{R} \\
\mathrm{R} \\
\mathrm{R} \\
\mathrm{R} \\
\mathrm{R} \\
\mathrm{R} \\
\mathrm{R} \\
\mathrm{R} \\
\mathrm{F} \\
\mathrm{F} \\
\\
\\
\mathrm{R} \\
\mathrm{R} \\
\mathrm{R} \\
\mathrm{R} \\
\mathrm{R} \\
\mathrm{F} \\
\mathrm{R} \\
\mathrm{R} \\
\mathrm{R} \\
\mathrm{R} \\
\mathrm{R} \\
\mathrm{R} \\
\mathrm{R} \\
\mathrm{R} \\
\mathrm{F} \\
\mathrm{R} \\
\mathrm{R} \\
\mathrm{T} \\
\mathrm{R} \\
\mathrm{R} \\
\mathrm{R} \\
\mathrm{R} \\
\mathrm{F}\end{array}$ & RW & R & $\begin{array}{l}\mathbf{F} \\
\mathrm{R}\end{array}$ & $\begin{array}{l}\mathbf{R} \\
\mathbf{R}\end{array}$ & $\begin{array}{l}\text { R } \\
\text { T } \\
\\
\text { R } \\
\\
\\
\text { D } \\
\text { D }\end{array}$ & $\begin{array}{l}\text { R } \\
\text { F } \\
\text { R } \\
\text { R } \\
\text { F } \\
\\
\text { R } \\
\text { R }\end{array}$ \\
\hline $20 \mathrm{X}, \mathrm{CC}$ & & & & & & & & & & & & & & & & & & & & & & & & & \\
\hline
\end{tabular}

Figure 5. Stratigraphic ranges of selected planktonic foraminifers at Hole 627B. 


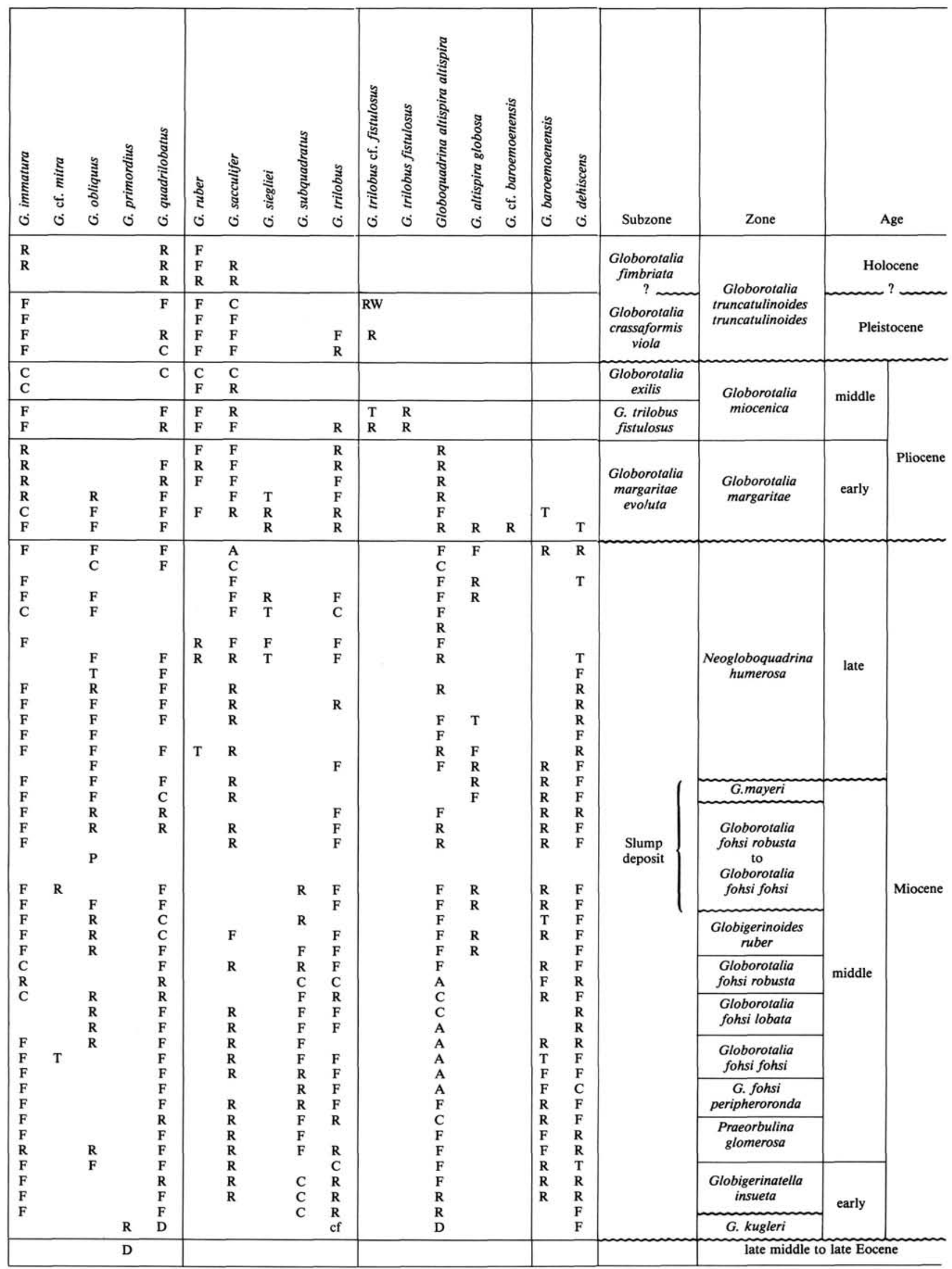

Figure 5 (continued). 


\begin{tabular}{|c|c|c|c|c|c|c|c|c|c|c|c|c|c|c|c|c|c|c|c|c|c|c|c|c|c|}
\hline $\begin{array}{c}\text { Hole 627B } \\
\text { Core-section } \\
\text { (interval in } \mathrm{cm} \text { ) }\end{array}$ & 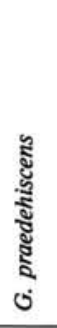 & $\begin{array}{l}\frac{a}{5} \\
\frac{5}{5} \\
0 \\
0\end{array}$ & 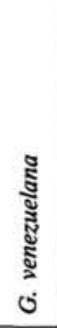 & 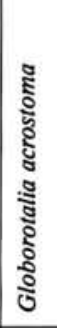 & 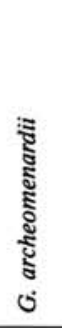 & 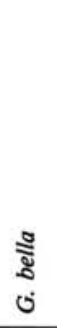 & 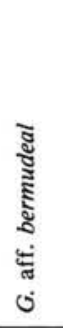 & 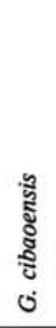 & 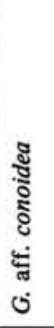 & 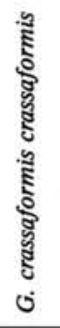 & 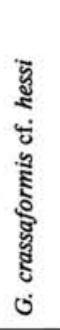 & 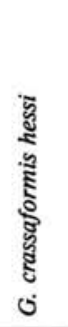 & 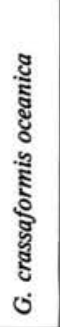 & 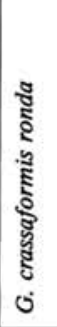 & 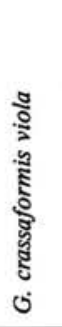 & 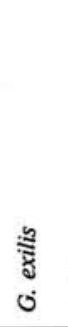 & ڤั & 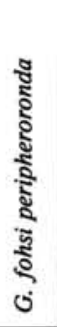 & 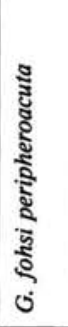 & $\underbrace{\frac{5}{5}}_{0}$ & 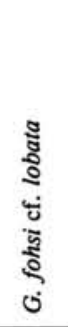 & $\begin{array}{l}5 \\
\frac{5}{5} \\
\frac{5}{5} \\
0\end{array}$ & 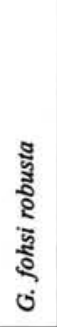 & 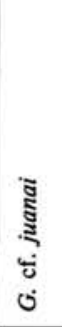 & 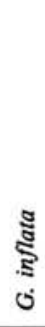 \\
\hline $\begin{array}{l}1 \mathrm{H}-1,25-27 \\
1 \mathrm{H}-2,25-27 \\
1 \mathrm{H}-4,25-27 \\
\end{array}$ & & & & & & & & & & & & RW & & & $\begin{array}{l}\mathrm{RW} \\
\mathrm{RW} \\
\mathrm{RW}\end{array}$ & & RW & & & & & & & & $\mathrm{F}$ \\
\hline $\begin{array}{l}1 \mathrm{H}, \mathrm{CC} \\
2 \mathrm{H}-3,25-27 \\
2 \mathrm{H}-6,25-27 \\
2 \mathrm{H}, \mathrm{CC}\end{array}$ & & & & & & & & & & $\begin{array}{l}\mathrm{R} \\
\mathrm{R} \\
\mathrm{R}\end{array}$ & R & & & $\begin{array}{l}\mathrm{R} \\
\mathrm{R}\end{array}$ & $\begin{array}{l}\mathrm{T} \\
\mathrm{T}\end{array}$ & $\begin{array}{l}\mathrm{RW} \\
\mathrm{RW} \\
\mathrm{RW}\end{array}$ & D & & & & & & & & D \\
\hline $\begin{array}{l}3 \mathrm{H}-3,25-27 \\
3 \mathrm{H}-5,25-27\end{array}$ & & & & & & & $\mathbf{R}$ & & & F & & & $\mathbf{R}$ & & $\begin{array}{l}\mathbf{R} \\
\mathbf{R}\end{array}$ & $\begin{array}{l}\mathrm{R} \\
\mathrm{R}\end{array}$ & & & & & & & & & \\
\hline $\begin{array}{l}3 \mathrm{H}-6,25-27 \\
3 \mathrm{H}, \mathrm{CC}\end{array}$ & & & & & & & & & & & & & $\mathbf{F}$ & $\mathbf{R}$ & & $\begin{array}{l}\mathbf{R} \\
\mathbf{R}\end{array}$ & & & & & & & & & \\
\hline $\begin{array}{l}4 \mathrm{H}-2,50-52 \\
4 \mathrm{H}, \mathrm{CC} \\
5 \mathrm{H}, \mathrm{CC} \\
6 \mathrm{H}, \mathrm{CC} \\
7 \mathrm{H}-2,25-27 \\
7 \mathrm{H}, \mathrm{CC} \\
\end{array}$ & & & $\mathrm{T}$ & & & & $\mathrm{T}$ & $\mathbf{R}$ & $\mathbf{T}$ & R & & & $F$ & & & $\mathrm{R}$ & D & & & & RW & & & $\begin{array}{l}\mathbf{R} \\
\mathbf{R} \\
\end{array}$ & \\
\hline $\begin{array}{l}8 \mathrm{H}-1,25-27 \\
8 \mathrm{H}-2,25-27 \\
8 \mathrm{H}-3,25-27 \\
8 \mathrm{H}-4,25-27 \\
8 \mathrm{H}-6,25-27 \\
8 \mathrm{H}, \mathrm{CC} \\
9 \mathrm{H}-2,25-27 \\
9 \mathrm{H}-3,25-27 \\
9 \mathrm{H}-4,25-27 \\
9 \mathrm{H}-6.25-27 \\
9 \mathrm{H}, \mathrm{CC} \\
10 \mathrm{H}-1,25-27 \\
10 \mathrm{H}-2,25-27 \\
10 \mathrm{H}-4,25-27 \\
10 \mathrm{H}-5,25-27 \\
10 \mathrm{H}, \mathrm{CC} \\
11 \mathrm{H}-1,25-27 \\
11 \mathrm{H}-2,25-27 \\
11 \mathrm{H}-3,25-27 \\
11 \mathrm{H}-4,25-27 \\
11 \mathrm{H}-5,25-27 \\
11 \mathrm{H}-6,25-27 \\
11 \mathrm{H}, \mathrm{CC} \\
12 \mathrm{H}-5,25-27 \\
12 \mathrm{H}-6,25-27 \\
12 \mathrm{H}-7,25-27 \\
12 \mathrm{H}, \mathrm{CC} \\
13 \mathrm{H}-1,25-27 \\
13 \mathrm{H}-3,25-27 \\
13 \mathrm{H}-4,25-27 \\
13 \mathrm{H}-5,25-27 \\
13 \mathrm{H}, \mathrm{CC} \\
14 \mathrm{H}-2,25-27 \\
14 \mathrm{H}-5,25-27 \\
14 \mathrm{H}, \mathrm{CC} \\
15 \mathrm{H}-2,28-30 \\
15 \mathrm{H}-5,25-27 \\
15 \mathrm{H}, \mathrm{C} \mathrm{C} \\
16 \mathrm{H}, \mathrm{CC} \\
17 \mathrm{X}-1,25-27 \\
17 \mathrm{X}-3,25-27 \\
17 \mathrm{X}, \mathrm{CC} \\
18 \mathrm{X}, \mathrm{CC} \\
19 \mathrm{X}-1,60-62 \\
19 \mathrm{X}, \mathrm{CC}\end{array}$ & $\mathbf{R}$ & $\begin{array}{l}\mathrm{T} \\
\mathrm{T}\end{array}$ & $\begin{array}{l} \\
\mathrm{R} \\
\\
\mathrm{R} \\
\mathrm{F} \\
\mathrm{R} \\
\mathrm{R} \\
\mathrm{F} \\
\mathrm{R} \\
\mathrm{R} \\
\mathrm{F} \\
\mathrm{F} \\
\mathrm{F} \\
\mathrm{P} \\
\mathrm{P} \\
\mathrm{R} \\
\mathrm{R} \\
\mathrm{R} \\
\mathrm{R} \\
\mathrm{R} \\
\mathrm{R} \\
\mathrm{R} \\
\mathrm{R} \\
\mathrm{R} \\
\mathrm{F} \\
\mathrm{F} \\
\mathrm{F} \\
\mathrm{F} \\
\mathrm{F} \\
\mathrm{F} \\
\mathrm{F} \\
\mathrm{F} \\
\mathrm{C} \\
\mathrm{R} \\
\mathrm{R} \\
\mathrm{R} \\
\mathrm{R} \\
\mathrm{F}\end{array}$ & $\begin{array}{l}\mathbf{R} \\
\mathbf{R}\end{array}$ & $\begin{array}{l}\mathbf{R} \\
\mathbf{R} \\
\mathrm{T}\end{array}$ & $\mathrm{T}$ & & $\begin{array}{l}\mathrm{R} \\
\mathrm{R} \\
\mathrm{R}\end{array}$ & $\begin{array}{l}\mathrm{P} \\
\mathrm{R}\end{array}$ & & & & & & & D & & $\begin{array}{l}\mathrm{R} \\
\mathrm{R} \\
\mathrm{R} \\
\\
\mathrm{R} \\
\mathrm{R}\end{array}$ & $\begin{array}{l}\text { R } \\
\text { R } \\
\text { R } \\
\text { R } \\
F \\
\text { R } \\
\text { F } \\
\text { R } \\
\text { R }\end{array}$ & $\begin{array}{l}\mathrm{F} \\
\mathrm{F} \\
\mathrm{R} \\
\\
\mathrm{R} \\
\mathrm{F}\end{array}$ & $\mathbf{R}$ & $\begin{array}{l}\mathbf{F} \\
\mathrm{F} \\
\mathrm{R} \\
\mathrm{P} \\
\mathrm{R} \\
\mathrm{F} \\
\\
\mathrm{R} \\
\mathrm{R}\end{array}$ & $\begin{array}{l}\mathbf{R} \\
\mathbf{R} \\
\mathbf{R}\end{array}$ & & D \\
\hline $20 \mathrm{X}, \mathrm{CC}$ & & & & & & & & & & & & & & & & & & & & & & & & & \\
\hline
\end{tabular}

Figure 5 (continued). 


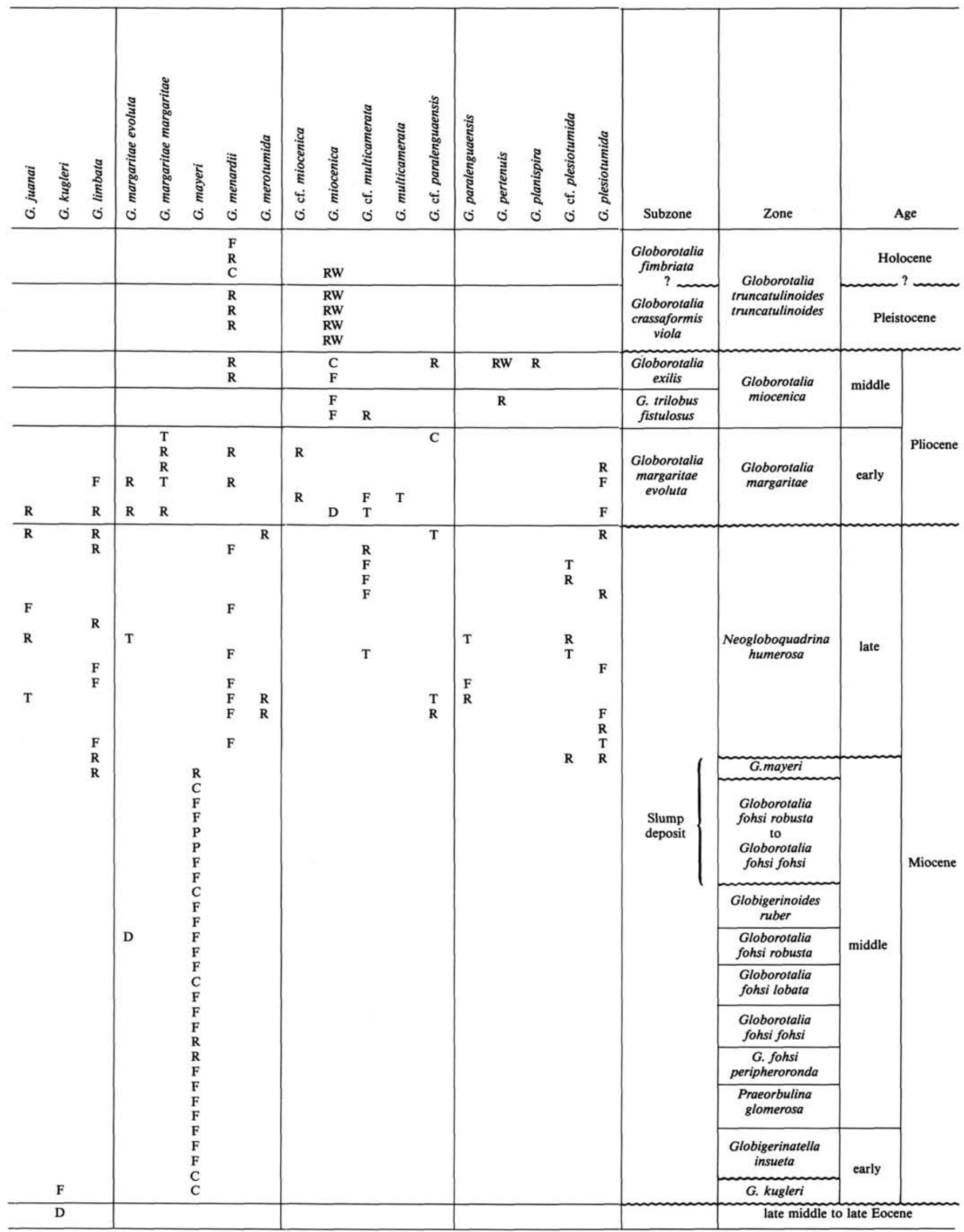

Figure 5 (continued). 


\begin{tabular}{|c|c|c|c|c|c|c|c|c|c|c|c|c|c|c|c|c|c|c|c|c|c|c|c|c|c|}
\hline $\begin{array}{c}\text { Hole 627B } \\
\text { Core-section } \\
\text { (interval in cm) }\end{array}$ & 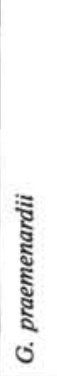 & 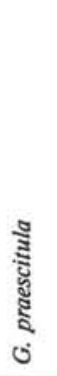 & 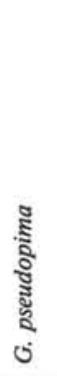 & 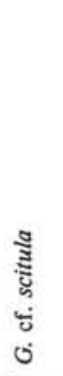 & $\begin{array}{l}\text { בี } \\
\text { ڤั } \\
ن\end{array}$ & 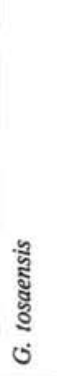 & 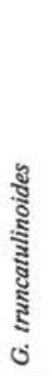 & 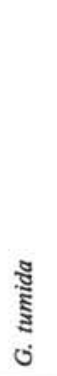 & 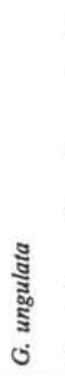 & 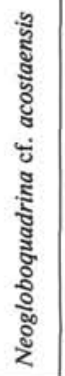 & 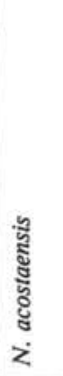 & $\begin{array}{l}5 \\
\frac{0}{0} \\
i \\
z\end{array}$ & 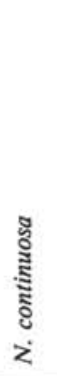 & 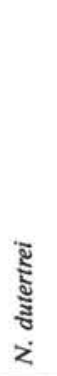 & 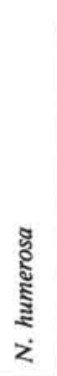 & 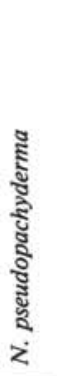 & 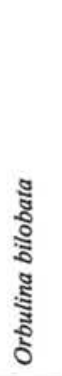 & 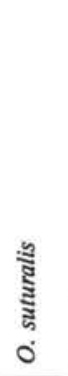 & 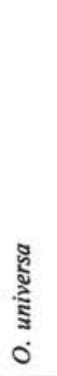 & 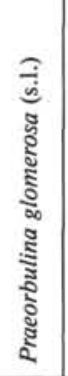 & 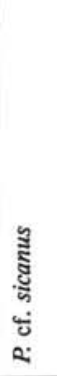 & 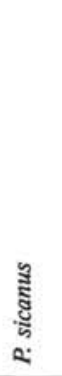 & 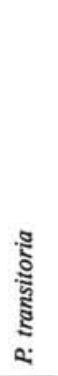 & 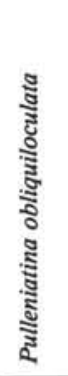 & 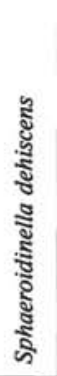 \\
\hline $\begin{array}{l}1 \mathrm{H}-1,25-27 \\
1 \mathrm{H}-2,25-27 \\
1 \mathrm{H}-4,25-27\end{array}$ & & & & & $\begin{array}{l}\mathbf{R} \\
\mathrm{T} \\
\mathrm{R}\end{array}$ & & $\begin{array}{l}\text { F } \\
\text { C } \\
\text { F }\end{array}$ & $\begin{array}{l}\mathrm{R} \\
\mathrm{R}\end{array}$ & $\begin{array}{l}R \\
R \\
F\end{array}$ & & & & & $\begin{array}{l}F \\
F \\
F\end{array}$ & & & & & $\begin{array}{l}\mathrm{F} \\
\mathrm{F} \\
\mathrm{F}\end{array}$ & & & & & $\begin{array}{l}\mathrm{R} \\
\mathrm{R}\end{array}$ & $\begin{array}{l}\mathrm{R} \\
\mathrm{R}\end{array}$ \\
\hline $\begin{array}{l}1 \mathrm{H}, \mathrm{CC} \\
2 \mathrm{H}-3,25-27 \\
2 \mathrm{H}-6,25-27 \\
2 \mathrm{H}, \mathrm{CC}\end{array}$ & & & $\mathbf{R}$ & & & $\begin{array}{l}\mathbf{R} \\
\mathrm{T} \\
\mathbf{R} \\
\end{array}$ & $\begin{array}{l}\mathrm{R} \\
\mathrm{D} \\
\mathrm{T} \\
\mathrm{R}\end{array}$ & & & & $\mathbf{R}$ & & & $\begin{array}{l}\mathrm{R} \\
\mathrm{R} \\
\mathrm{T} \\
\mathrm{F}\end{array}$ & & & & & $\begin{array}{l}\mathrm{F} \\
\mathrm{F} \\
\mathrm{F} \\
\mathrm{F}\end{array}$ & & & & & $\begin{array}{l}\mathrm{R} \\
\mathrm{D}\end{array}$ & $\begin{array}{l}\mathrm{R} \\
\mathrm{R} \\
\mathrm{T} \\
\end{array}$ \\
\hline $\begin{array}{l}3 \mathrm{H}-3,25-27 \\
3 \mathrm{H}-5,25-27\end{array}$ & & & $F$ & & & & & & & $T$ & & & & $\mathrm{~T}$ & $\begin{array}{l}\mathrm{R} \\
\mathrm{R}\end{array}$ & & $\mathbf{R}$ & & $\begin{array}{l}\mathrm{C} \\
\mathrm{F}\end{array}$ & & & & & & $T$ \\
\hline $\begin{array}{l}3 \mathrm{H}-6,25-27 \\
3 \mathrm{H}, \mathrm{CC}\end{array}$ & & & & & & & & & & & & & & & $\begin{array}{l}\mathrm{R} \\
\mathrm{R}\end{array}$ & & $\mathbf{R}$ & & $\begin{array}{l}\mathrm{C} \\
\mathrm{C}\end{array}$ & & & & & & $\mathbf{R}$ \\
\hline $\begin{array}{l}4 \mathrm{H}-2,50-52 \\
4 \mathrm{H}, \mathrm{CC} \\
5 \mathrm{H}, \mathrm{CC} \\
6 \mathrm{H}, \mathrm{CC} \\
7 \mathrm{H}-2,25-27 \\
7 \mathrm{H}, \mathrm{CC}\end{array}$ & & & $\mathbf{R}$ & & $\begin{array}{l}\mathrm{R} \\
\mathrm{R}\end{array}$ & & $\begin{array}{l}\text { D } \\
\text { D }\end{array}$ & & & & $\begin{array}{l}\mathrm{R} \\
\mathrm{R} \\
\mathrm{R} \\
\mathrm{R} \\
\mathrm{R}\end{array}$ & $\mathbf{R}$ & & D & $\begin{array}{l}\mathrm{R} \\
\mathrm{R} \\
\mathrm{R}\end{array}$ & & $\begin{array}{l}\mathrm{R} \\
\mathrm{R} \\
\mathrm{R} \\
\mathrm{R} \\
\mathrm{R} \\
\mathrm{R}\end{array}$ & & $\begin{array}{l}\text { A } \\
\text { C } \\
\text { C } \\
\text { C } \\
\text { F } \\
\text { C }\end{array}$ & & & & & D & $\begin{array}{l}\mathrm{C} \\
\mathrm{R}\end{array}$ \\
\hline $\begin{array}{l}8 \mathrm{H}-1,25-27 \\
8 \mathrm{H}-2,25-27 \\
8 \mathrm{H}-3,25-27 \\
8 \mathrm{H}-4,25-27 \\
8 \mathrm{H}-6,25-27 \\
8 \mathrm{H}, \mathrm{CC} \\
9 \mathrm{H}-2,25-27 \\
9 \mathrm{H}-3,25-27 \\
9 \mathrm{H}-4,25-27 \\
9 \mathrm{H}-6.25-27 \\
9 \mathrm{H}, \mathrm{CC} \\
10 \mathrm{H}-1,25-27 \\
10 \mathrm{H}-2,25-27 \\
10 \mathrm{H}-4,25-27 \\
10 \mathrm{H}-5,25-27 \\
10 \mathrm{H}, \mathrm{CC} \\
11 \mathrm{H}-1,25-27 \\
11 \mathrm{H}-2,25-27 \\
11 \mathrm{H}-3,25-27 \\
11 \mathrm{H}-4,25-27 \\
11 \mathrm{H}-5,25-27 \\
11 \mathrm{H}-6,25-27 \\
11 \mathrm{H}, \mathrm{CC} \\
12 \mathrm{H}-5,25-27 \\
12 \mathrm{H}-6,25-27 \\
12 \mathrm{H}-7,25-27 \\
12 \mathrm{H}, \mathrm{CC} \\
13 \mathrm{H}-1,25-27 \\
13 \mathrm{H}-3,25-27 \\
13 \mathrm{H}-4,25-27 \\
13 \mathrm{H}-5,25-27 \\
13 \mathrm{H}, \mathrm{CC} \\
14 \mathrm{H}-2,25-27 \\
14 \mathrm{H}-5,25-27 \\
14 \mathrm{H}, \mathrm{CC} \\
15 \mathrm{H}-2,28-30 \\
15 \mathrm{H}-5,25-27 \\
15 \mathrm{H}, \mathrm{C} \mathrm{C} \\
16 \mathrm{H}, \mathrm{CC} \\
17 \mathrm{X}-1,25-27 \\
17 \mathrm{X}-3,25-27 \\
17 \mathrm{X}, \mathrm{CC} \\
18 \mathrm{X}, \mathrm{CC} \\
19 \mathrm{X}-1,60-62 \\
19 \mathrm{X}, \mathrm{CC}\end{array}$ & $\begin{array}{l}R \\
R \\
R \\
F \\
P \\
P \\
F \\
R \\
R \\
F \\
F \\
R \\
R \\
R \\
R \\
R \\
R \\
R \\
R \\
T\end{array}$ & $\begin{array}{l}\mathrm{F} \\
\mathrm{R} \\
\mathrm{R} \\
\mathrm{T} \\
\mathrm{R} \\
\mathrm{T}\end{array}$ & 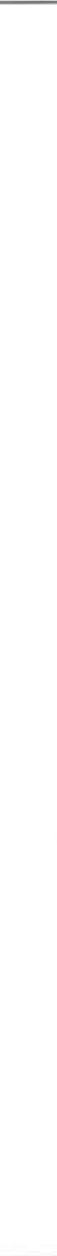 & $T$ & $\begin{array}{l}\mathrm{R} \\
\mathrm{R} \\
\mathrm{R}\end{array}$ & & D & & & & $\begin{array}{l}\mathrm{F} \\
\mathrm{F} \\
\mathrm{R} \\
\mathrm{R} \\
\mathrm{T} \\
\mathrm{F} \\
\mathrm{R} \\
\mathrm{R} \\
\mathrm{T} \\
\mathrm{F} \\
\mathrm{R} \\
\mathrm{R} \\
\mathrm{R} \\
\mathrm{R}\end{array}$ & 更 & $\begin{array}{l}\mathrm{R} \\
\mathrm{R} \\
\mathrm{R} \\
\mathrm{R} \\
\\
\\
\\
\mathrm{R} \\
\mathrm{R} \\
\mathrm{R} \\
\mathrm{R} \\
\mathrm{R} \\
\mathrm{R} \\
\\
\mathrm{R} \\
\mathrm{R} \\
\mathrm{R} \\
\mathrm{R} \\
\mathrm{R} \\
\mathrm{R} \\
\mathrm{R} \\
\mathrm{R} \\
\mathrm{R} \\
\mathrm{T} \\
\mathrm{R} \\
\mathrm{R} \\
\mathrm{R} \\
\mathrm{R} \\
\mathrm{R}\end{array}$ & & $\begin{array}{l}\mathrm{R} \\
\mathrm{R} \\
\mathrm{T} \\
\\
\mathrm{R} \\
\mathrm{R} \\
\\
\\
\mathrm{T}\end{array}$ & $\mathrm{T}$ & $\begin{array}{l}\mathrm{F} \\
\mathrm{F} \\
\mathrm{R} \\
\mathrm{F} \\
\mathrm{R} \\
\mathrm{F} \\
\mathrm{F} \\
\mathrm{F} \\
\mathrm{F} \\
\mathrm{F} \\
\mathrm{F} \\
\mathrm{F} \\
\mathrm{F} \\
\mathrm{F} \\
\mathrm{R} \\
\mathrm{F} \\
\mathrm{R} \\
\mathrm{R} \\
\mathrm{R} \\
\\
\mathrm{R} \\
\mathrm{R} \\
\mathrm{R} \\
\mathrm{R} \\
\mathrm{R} \\
\mathrm{R} \\
\mathrm{R} \\
\mathrm{R} \\
\mathrm{F} \\
\mathrm{F} \\
\end{array}$ & $\begin{array}{l} \\
\\
\\
\\
\\
\\
\\
\\
\end{array}$ & $\begin{array}{l}\mathrm{C} \\
\mathrm{A} \\
\mathrm{C} \\
\mathrm{C} \\
\mathrm{C} \\
\mathrm{C} \\
\mathrm{C} \\
\mathrm{C} \\
\mathrm{A} \\
\mathrm{A} \\
\mathrm{C} \\
\mathrm{C} \\
\mathrm{A} \\
\mathrm{A} \\
\mathrm{A} \\
\mathrm{C} \\
\mathrm{C} \\
\mathrm{C} \\
\mathrm{A} \\
\mathrm{A} \\
\mathrm{P} \\
\mathrm{P} \\
\mathrm{F} \\
\mathrm{F} \\
\mathrm{F} \\
\mathrm{R} \\
\mathrm{F} \\
\mathrm{F} \\
\mathrm{F} \\
\mathrm{F} \\
\mathrm{A} \\
\mathrm{C} \\
\mathrm{A} \\
\mathrm{A} \\
\mathrm{C} \\
\mathrm{F} \\
\mathrm{F} \\
\end{array}$ & $\begin{array}{l}\mathrm{F} \\
\mathrm{F} \\
\mathrm{R}\end{array}$ & $\mathbf{R}$ & $\begin{array}{l}\text { R } \\
\text { F } \\
\text { F } \\
\text { F } \\
\text { C } \\
\text { F }\end{array}$ & $\begin{array}{l}\mathrm{T} \\
\mathrm{F}\end{array}$ & & \\
\hline $20 \mathrm{X}, \mathrm{CC}$ & & & & & & & & & & & & & & & & & & & & & & & & & \\
\hline
\end{tabular}

Figure 5 (continued). 


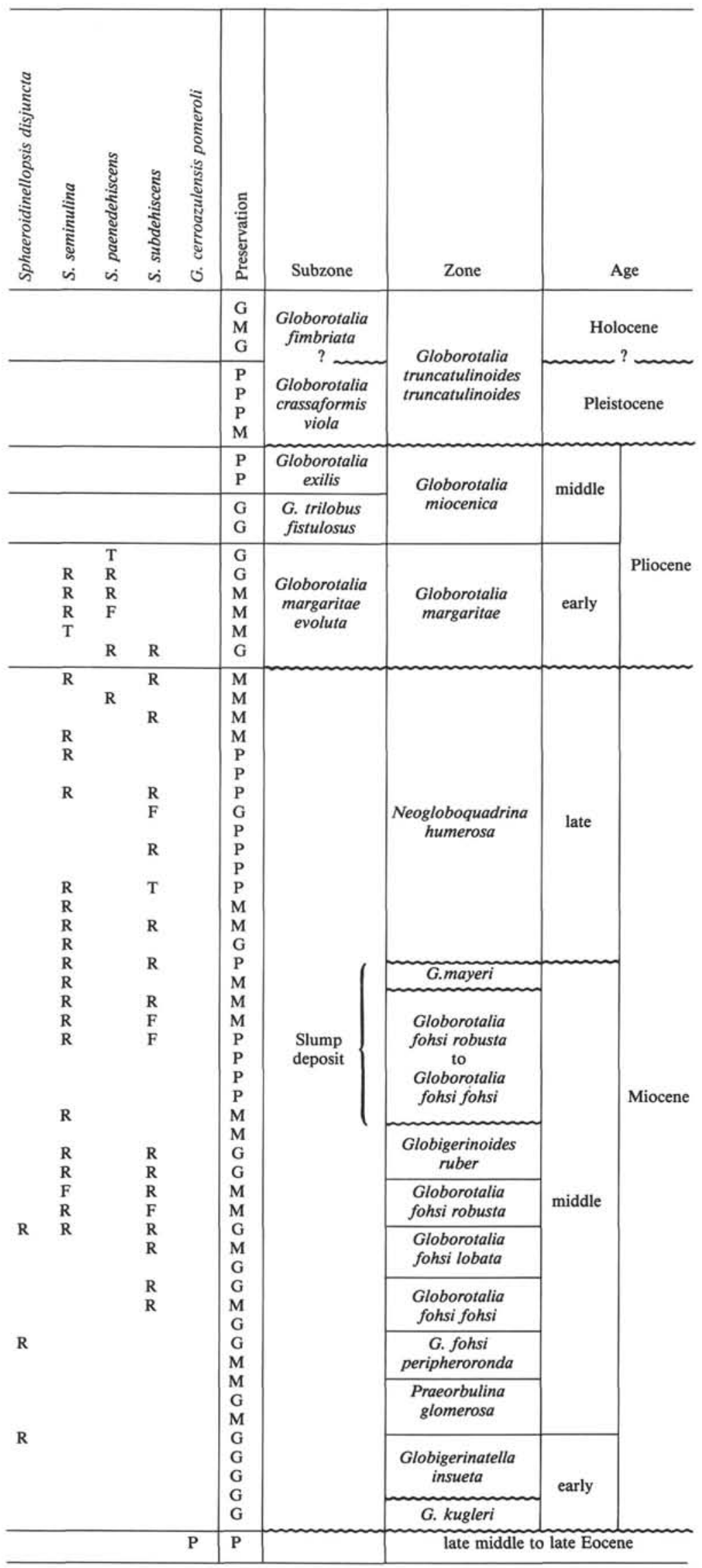

Figure 5 (continued). 
Sample 101-627B-11H-1, 25-27 cm, located near the top of the slump, correlates with the late middle Miocene Globorotalia mayeri Zone, based on the presence of the nominate taxon and Globigerina nepenthes Todd without Globigerinoides subquadratus (Fig. 5). This suggests that the latest middle Miocene Globorotalia menardii Zone is missing (Fig. 5). The break may be associated with the emplacement of the slump deposit. However, the absence of sediments that correlate with the Globorotalia menardii Zone in the repeated section below suggests the presence of an unconformity at or near the middle/upper Miocene boundary (Fig. 5).

Samples 101-627B-11H-2, 25-27 cm, through 101-627B-11H4, 25-27 cm, contain G. fohsi fohsi, G. fohsi lobata, and $G$. fohsi robusta (Fig. 5). According to Bolli and Saunders (1985), the stratigraphic ranges of $G$. fohsi fohsi and $G$. fohsi robusta do not overlap. Thus, it appears that either mixing and/or reworking occurred during slumping. As a result, these sediments correlate with the undifferentiated Globorotalia fohsi fohsi to Globorotalia fohsi robusta Zones (Fig. 5). Samples 101-627B$11 \mathrm{H}-5,25-27 \mathrm{~cm}$, and 101-627B-11H-6, 25-27 cm, are composed of lithified clasts and chalky oozes that contain few identifiable foraminifers (Fig. 5). However, the presence of rare specimens of $G$. praemenardii indicates that these sediments also correlate with the undifferentiated Globorotalia fohsi fohsi to Globorotalia fohsi robusta zones (Fig. 5; Bolli and Saunders, 1985). The absence of the Globigerinoides ruber Zone probably is associated with the emplacement of the slump, as this zone is represented in the in-situ section described next (Fig. 5).

A middle Miocene age (lower Globorotalia fohsi lobata Zone) was assigned to the sediments in Section 101-627B-11H, CC and Sample 101-627B-12H-5, 25-27 cm, based on the co-occurrence of $G$. fohsi lobata and G. fohsi fohsi (Fig. 5; Bolli and Saunders, 1985). Sample 101-627B-12H-5, 25-27 cm, is located $76 \mathrm{~cm}$ above an abrupt change (Sample 101-627B-12H-5, $103 \mathrm{~cm}$ ) from darker-colored, laminated soft oozes at the base of the slump to lighter-colored, unlaminated, stiff oozes below this slump (Austin, Schlager, et al., 1986). This change defines the contact between lithologic Subunits IA and IB and also correlates with the base of seismic sequence B, as calculated using the traveltimes obtained from the LBB 18 site-survey seismic line (Austin, Schlager, et al., 1986). This repeated biostratigraphic sequence does not appear to be due to double-coring of the section but resulted from a late middle Miocene slumping event at Site 627.

The interval between Sample 101-627B-12H-6, 25-27 cm (109.25 mbsf) and Section 101-627B-12H, CC was assigned to the late middle Miocene Globigerinoides ruber Zone (Fig. 5), based on the presence of Globigerinoides subquadratus and the absence of members of the Globorotalia fohsi group (Fig. 5). Shipboard studies, which reported a correlation with the Globorotalia mayeri Zone, used the zonation of Stainforth et al. (1975), in which the Globigerinoides ruber Zone of Bolli (1957) is combined into the Globorotalia mayeri Zone. Here, the zonation of Bolli and Saunders (1985) is used, in which the authors reaffirm the validity of the Globigerinoides ruber Zone in the Caribbean region.

The presence of $G$. fohsi peripheroacuta Blow and Banner and $G$. fohsi fohsi in all five samples in the interval between Sample 101-627B-13H-5, 25-27 cm, and Section 101-627B-14H, $\mathrm{CC}$ indicates the presence of the early middle Miocene Globorotalia fohsi fohsi Zone (Fig. 5). On board the JOIDES Resolution, time constraints only allowed examination of Section 101$627 \mathrm{~B}-13 \mathrm{H}, \mathrm{CC}$ from this interval. The presence of very rare specimens of $G$. fohsi lobata in that sample was the basis on which Core 101-627B-13H was correlated with the Globorotalia fohsi lobata Zone (Austin, Schlager, et al., 1986). Based on the absence of this species in the interval above and below Section 101-627B-13H, CC, as shown by shore-based studies, the speci- mens of $G$. fohsi lobata in that sample were considered to be downhole contaminants. Thus, the zonal assignment was changed here to Globorotalia fohsi fohsi Zone (Fig. 5).

Sediments of the early middle Miocene Globorotalia fohsi peripheroronda Zone were first observed in Sample 101-627B$15 \mathrm{H}-2,28-30 \mathrm{~cm}$, based on the absence of $G$. fohsi fohsi. This zone occurs down to the level of Sample 101-627B-15H-5, 25-27 $\mathrm{cm}$, where Orbulina universa first appears (Fig. 5).

The absence of Orbulina universa in Section 101-627B-15H, CC indicates the Praeorbulina glomerosa Zone (Fig. 5). Specimens of $P$. glomerosa s.l. are present in moderate numbers (Fig. 5). The presence of Orbulina suturalis indicates the uppermost part of the zone (Fig. 5; Bolli and Saunders, 1985). The FO of Praeorbulina glomerosa s.1. in Sample 101-627B-17X-1, 25-27 $\mathrm{cm}$, marks the base of the zone (Fig. 5). As used here, this base is probably slightly below the level that one would choose if the FO of $P$. glomerosa glomerosa was used as the zonal datum, as suggested by Bolli and Saunders (1985). At Sites 627 and 628, subspecies of $P$. glomerosa were difficult to identify consistently because, in most cases, the arrangement and shapes of the supplementary apertures were obscured by overgrowth and/or infilling with carbonate material.

Lower Miocene sediments are first encountered in Sample 101-627B-17X-3, 25-27 cm (Fig. 5). The absence of Praeorbulina glomerosa s.l. and Catapsydrax dissimilis suggests the presence of the Globigerinatella insueta Zone (Fig. 5). The base of this zone is placed at the level of Sample 101-627B-19X-1, 60$62 \mathrm{~cm}$ (Fig. 5).

The sediments in Sample 101-627B-19X, CC correlate with the earliest Miocene Globorotalia kugleri Zone, based on the presence of the nominate taxon (Fig. 5). It may be that an unconformity is present between Sample 101-627B-19X-1, 60-62 $\mathrm{cm}$, and the core-catcher sample in which the Catapsydrax dissimilis Zone and the Catapsydrax stainforthi Zone are missing, or it may be that these zones are present in the intervening section but are very thin. The absence of sediments that correlate with these zones at Site 628 , located approximately $11.1 \mathrm{~km}$ upslope of Site 627 , may support the interpretation of an unconformity.

Recovery in Core 101-627B-20X was very poor and consisted of approximately $1 \mathrm{~m}$ of caved(?) Globorotalia kugleri Zone sediments (Sample 101-627B-20X-1, 25-27 cm) and the corecatcher sample, which contained a chert pebble and carbonate material. The chert pebble contained the middle-upper Eocene marker species Turborotalia cerroazulensis pomeroli (Toumarkine and Bolli), while the carbonate material contained foraminifers that indicate the earliest Miocene Globorotalia kugleri Zone (Austin, Schlager, et al., 1986). Cores 101-627B-21X through 101-627B-23X are Eocene in age, based on planktonic foraminifers, with radiolarians and calcareous nannofossils corroborating this age assignment in Cores 101-627B-21X and 101-627B23X, respectively (Austin, Schlager, et al., 1986; Palmer, this volume; Watkins and Verbeek, this volume). Thus, a major unconformity may be present at Site 627 with most or all of the Oligocene section missing (Fig. 5).

\section{Site 628}

Site 628 is located $11.1 \mathrm{~km}$ upslope of Site 627 , approximately at the present-day toe-of-slope, and serves as the middle leg of the LBB transect (Fig. 1). One hole was drilled in $966 \mathrm{~m}$ of water. Thirty-two cores penetrated to $298.4 \mathrm{mbsf}$, and 216.9 $\mathrm{m}$ of sediment was recovered $(72.7 \%$ recovery). Neogene sediments are present down to $136.6 \mathrm{mbsf}$. Total recovery of Neogene sediments was $133.4 \mathrm{~m}(97.4 \%)$ (Austin, Schlager, et al., 1986).

The age of the sediments in Cores $101-628-1 \mathrm{H}$ and 101-628A$2 \mathrm{H}$ was difficult to determine precisely because of reworking and possible downhole contamination. The sediments in Sam- 
ples 101-628A-1H-2, 25-27 cm, and 101-628A-1H, CC were assigned a Pleistocene-Holocene age (Globorotalia truncatulinoides truncatulinoides Zone), based on the presence of the zonal marker species (Fig. 6). Specimens of $G$. miocenica, Globigerinoides extremus, and $G$. trilobus fistulosus, all of which became extinct in the Pliocene, are considered reworked (Fig. 6). In addition, very rare specimens of Globorotalia fohsi fohsi are present and indicate reworking from middle Miocene sediments (Fig. 6).

Also present in Core 101-628A-1H are specimens of $G$. crassaformis viola, $G$. crassaformis hessi, and $G$. flexuosa Koch, species whose last appearance datums are Pleistocene subzonal indicators (Fig. 2). However, the presence of extremely rare specimens of $G$. ungulata indicates that these sediments occur in the Holocene Globorotalia fimbriata Subzone (Figs. 2 and 6). I believe that Pleistocene sediments also were reworked into this level and, along with the other reworked foraminifers, compose most of the assemblage.

The highest occurrence of Pliocene sediments is in Sample $101-628 \mathrm{~A}-2 \mathrm{H}-3,25-27 \mathrm{~cm}$, based on the absence of $G$. truncatulinoides truncatulinoides (Fig. 6). A middle Pliocene age (Globorotalia miocenica Zone) was assigned to the interval between this sample and Section 101-628A-3H, CC, based on the presence of the nominate taxon and $G$. exilis (Fig. 6). The absence of Globigerinoides trilobus fistulosus suggests a correlation with the Globorotalia exilis Subzone (Figs. 2, 6). This subzonal assignment was supported by the absence of $G$. multicamerata, $G$. pertenuis and Globoquadrina altispira altispira Cushman and Jarvis (Fig. 6; Bolli and Saunders, 1985).

Section 101-628A-4H, CC contains foraminifers referable to Globorotalia sp. cf. G. margaritae margaritae without $G$. miocenica or G. exilis, which suggests the presence of the early Pliocene Globorotalia margaritae Zone (Fig. 6). Well-developed specimens of G. margaritae margaritae are present in Section 101$628 \mathrm{~A}-5 \mathrm{H}, \mathrm{CC}$ and clearly indicate an early Pliocene age (Globorotalia margaritae Zone) for these sediments (Fig. 6). Sediments that correlate with this zone extend down to the level of Section 101-628A-8H, CC, where G. margaritae margaritae first occurs (Fig. 6). Well-developed specimens of $G$. margaritae evoluta were not observed and, thus, no subzones were assigned (Fig. 6).

Uppermost Miocene sediments (Neogloboquadrina humerosa Zone) occur in Sample 101-628A-9H-3, 25-27 cm, based on the absence of Globorotalia margaritae margaritae (Fig. 6). Specimens of Neogloboquadrina humerosa occur sporadically and in small numbers at Hole 628A (Fig. 6). Its FO in Sample 101$628 \mathrm{~A}-11 \mathrm{H}-3,25-27 \mathrm{~cm}$, is above the FO of Candeina nitida and Globorotalia plesiotumida in Sample 101-628A-13H-3, 25-27 cm (Fig. 6). According to Bolli and Saunders (1985), these datums occur above the first evolutionary appearance of Neogloboquadrina humerosa (i.e., base of Neogloboquadrina humerosa Zone). Thus, the presence of $N$. humerosa in Sample 101-628A-11H-3, $25-27 \mathrm{~cm}$, is not an evolutionary event and is above the lower limit of the zone, which was tentatively placed at the level of Sample 101-628A-13H-3, 25-27 cm (Fig. 6). According to Bolli and Saunders (1985), the presence of Candeina nitida indicates the upper part of the zone.

The middle/late Miocene boundary at Site 628 is marked by an unconformity (Figs. 2 and 6). Section 101-628A-13H, CC contains Globorotalia mayeri and Globigerina nepenthes, which indicates the late middle Miocene Globorotalia mayeri Zone (Fig. 6). This suggests that the early late Miocene Neogloboquadrina acostaensis Zone and the latest middle Miocene Globorotalia menardii Zone are missing (Figs. 2 and 6). A single specimen of $G$. sp. cf. $G$. fohsi lobata and a few specimens of $G$. praemenardii were present in Section 101-628A-13H, CC, but were considered as reworked (Fig. 6). This inference was supported by the presence of common platform-derived material.
The LOs of $G$. fohsi lobata and $G$. fohsi robusta are in Sample $101-628 \mathrm{~A}-14 \mathrm{H}-3,25-27 \mathrm{~cm}$, which indicates that the top of the Globorotalia fohsi robusta Zone occurs at this level (Fig. 6). Thus, it appears that the Globigerinoides ruber Zone is missing (Fig. 6). Sediments of the Globorotalia fohsi robusta Zone extend down to the level of Sample 101-628A-15H-3, 25-27 cm, where $G$. fohsi robusta first occurs (Fig. 6). The presence of rare specimens of $G$. fohsi fohsi in this sample indicates that either reworking of this species or caving of $G$. fohsi robusta occurred (Fig. 6; Bolli and Saunders, 1985). In the latter case, the sediments should correlate with the Globorotalia fohsi lobata Zone (Fig. 2). However, based on the large amount of reworking seen in the Bahamas during Leg 101, the presence of reworked specimens of $G$. fohsi fohsi in the Pleistocene, and the paucity of this species in Sample 101-628A-15H-3, 25-27 cm, the specimens are considered as reworked (Fig. 6).

Sediments that correlate with the lower(?) Globorotalia fohsi lobata Zone were identified in Section 101-628A-15H, CC, based on the presence of the nominate taxon and $G$. fohsi fohsi without $G$. fohsi robusta (Fig. 6).

A major unconformity encompassing much of the middle Miocene and all of the early Miocene is present at Site 628 (Fig. $6)$. This is supported by the presence of upper Oligocene sediments (Globigerina ciperoensis Zone) in Sample 101-628A-16H$3,25-27 \mathrm{~cm}$ (Fig. 6). Species present that indicate this zone include $G$. ciperoensis ciperoensis Bolli, G. ciperoensis fariasi Bermudez, $G$. angulisuturalis Bolli, and $G$. officinalis Subbotina (Fig. 6; Bolli and Saunders, 1985).

\section{Site 630}

Hole 630A, which serves as the shallow end of the Little Bahama Bank transect, is located on an interfluve in the gullied upper part of the slope in $807 \mathrm{~m}$ of water (Austin, Schlager, et al., 1986). The site was drilled with the HPC to a depth of 172.1 mbsf (Cores 101-630A-1H to 101-630A-18H) and then was drilled using the XCB to a depth of 250.3 mbsf (Cores 101-630A-19H to $101-630 \mathrm{~A}-26 \mathrm{X}$ ). A total of $220.3 \mathrm{~m}$ of Neogene sediments was recovered $(88 \%$ recovery).

The sediments in Core 101-630A-1H correlate with the Pleistocene-Holocene Globorotalia truncatulinoides truncatulinoides Zone, based on the presence of the nominate taxon (Fig. 7). The fauna in Sample 101-630A-1H-3, 25-27 cm, is composed mainly of reworked Miocene-Pliocene species and contains few in-situ specimens (Fig. 7). Calcareous nannofossils indicate a Holocene age $(\mathrm{CN} 15)$ for this level, and that age assignment is used here (Watkins and Verbeek, this volume). Samples 101-630A-1H-4, $25-27 \mathrm{~cm}$, and 101-630A-1H, CC contain much smaller numbers of reworked foraminifers (Fig. 7). These assemblages also contain specimens of apparently in-situ G. crassaformis viola (Fig. 7). On this basis, the sediments in these samples were correlated with the lower Pleistocene Globorotalia crassaformis viola Subzone and appear to lie unconformably below the Holocene sediments in Sample 101-630A-1H-3, 25-27 cm (Fig. 7).

A second unconformity may separate the lower Pleistocene from the middle Pliocene at Hole 630A (Fig. 7). The presence of $G$. miocenica and $G$. exilis without Globigerinoides trilobus fistulosus in Samples 101-630A-2H-4, 25-27 cm, and 101-630A$2 \mathrm{H}, \mathrm{CC}$ indicate that these sediments correlate with the Globorotalia miocenica Zone (Globorotalia exilis Subzone) (Fig. 7). The nominate taxon of the Globigerinoides trilobus fistulosus Subzone is present in Samples 101-630A-3H-4, 25-27 cm, through 101-630A-4H-3, 25-27 cm (Fig. 7). The sediments in Sections 101-630A-4H, CC through 101-630A-5H, CC also correlate with this subzone (despite the absence of the index species) on the basis of superposition (Fig. 7). The transition from early to middle Pliocene at Hole $630 \mathrm{~A}$ is characterized by the presence of forms that compare favorably with $G$. miocenica and $G$. margaritae margaritae, but cannot be assigned to these 


\begin{tabular}{|c|c|c|c|c|c|c|c|c|c|c|c|c|c|c|c|c|c|c|c|c|c|c|c|c|c|c|c|}
\hline $\begin{array}{c}\text { Hole 628A } \\
\text { Core-section } \\
\text { (interval in cm) }\end{array}$ & 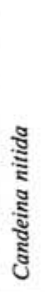 & 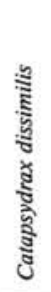 & 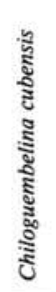 & 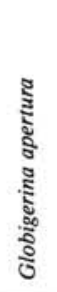 & 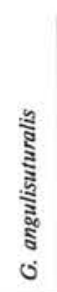 & 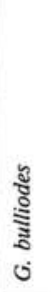 & 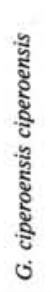 & 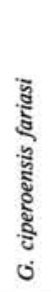 & 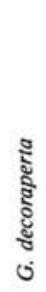 & $\begin{array}{l}\frac{\pi}{5} \\
0\end{array}$ & 仓ั & 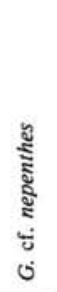 & 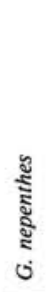 & 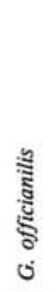 & 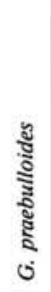 & 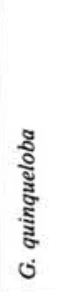 & $\begin{array}{l}\text { है } \\
\text { हु } \\
\text { है } \\
\dot{0}\end{array}$ & $\begin{array}{c}\text { ปूँ } \\
\text { : } \\
\text { Е } \\
ن\end{array}$ & 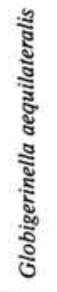 & $\begin{array}{l}\text { ठूँ } \\
0 \\
0\end{array}$ & 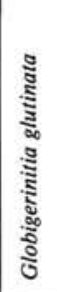 & 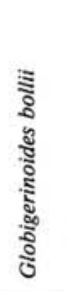 & 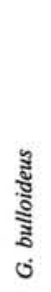 & 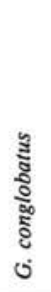 & 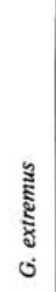 & 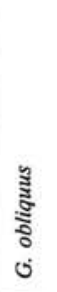 & है \\
\hline $\begin{array}{l}1 \mathrm{H}-2,25-27 \\
1 \mathrm{H}, \mathrm{CC}\end{array}$ & $\mathbf{R}$ & & & & & & & & & & & & & & & $\mathbf{R}$ & $\begin{array}{l}R \\
R\end{array}$ & & $\begin{array}{l}\mathrm{F} \\
\mathrm{F}\end{array}$ & & $\begin{array}{l}\mathrm{R} \\
\mathrm{R}\end{array}$ & & & $\begin{array}{l}\mathbf{R} \\
\mathrm{F}\end{array}$ & $\begin{array}{l}\mathrm{RW} \\
\mathrm{RW}\end{array}$ & & \\
\hline $\begin{array}{l}2 \mathrm{H}-3,25-27 \\
2 \mathrm{H}, \mathrm{CC} \\
3 \mathrm{H}-3,25-27 \\
3 \mathrm{H}, \mathrm{CC}\end{array}$ & $\begin{array}{l}\mathrm{R} \\
\mathrm{R}\end{array}$ & & & & & & & & $\mathbf{R}$ & & & $\begin{array}{l}\mathrm{R} \\
\mathrm{R}\end{array}$ & & & & $\begin{array}{l}F \\
F\end{array}$ & & $\mathbf{R}$ & $\begin{array}{l}\mathrm{R} \\
\mathrm{R} \\
\mathrm{R} \\
\mathrm{F}\end{array}$ & & $\begin{array}{l}\mathrm{R} \\
\mathrm{R} \\
\mathrm{F} \\
\mathrm{R}\end{array}$ & & & $\begin{array}{l}R \\
R \\
F \\
F\end{array}$ & $\begin{array}{l}\mathrm{R} \\
\mathrm{R} \\
\end{array}$ & & \\
\hline $\begin{array}{l}4 \mathrm{H}, \mathrm{CC} \\
5 \mathrm{H}-3,25-27 \\
5 \mathrm{H}, \mathrm{CC} \\
6 \mathrm{H}-3,25-27 \\
6 \mathrm{H}, \mathrm{CC} \\
7 \mathrm{H}-3,25-27 \\
7 \mathrm{H}, \mathrm{CC} \\
8 \mathrm{H}-3,25-27 \\
8 \mathrm{H}, \mathrm{CC}\end{array}$ & $\begin{array}{l}R \\
\\
R \\
R \\
R \\
R \\
R \\
R\end{array}$ & & & $\mathbf{R}$ & & $\begin{array}{l}R \\
R \\
R \\
R \\
R \\
R \\
F\end{array}$ & & & $\begin{array}{l}\mathrm{R} \\
\mathrm{R} \\
\mathrm{F} \\
\mathrm{F} \\
\mathrm{F} \\
\mathrm{F} \\
\mathrm{F}\end{array}$ & & $\begin{array}{l}\mathrm{R} \\
\mathrm{R} \\
\mathrm{R}\end{array}$ & & $\begin{array}{l}\mathrm{R} \\
\mathrm{F} \\
\mathrm{R} \\
\mathrm{C} \\
\mathrm{R} \\
\mathrm{R} \\
\mathrm{F} \\
\mathrm{F}\end{array}$ & & & $\mathbf{R}$ & & & $\begin{array}{l}\text { C } \\
\text { C } \\
\text { C } \\
\text { C } \\
\text { F } \\
\text { R } \\
\text { F } \\
\text { F } \\
\text { R }\end{array}$ & $\begin{array}{l}\mathrm{F} \\
\mathrm{F} \\
\mathrm{F} \\
\mathrm{F} \\
\mathrm{F} \\
\mathrm{F} \\
\mathrm{F} \\
\mathrm{F}\end{array}$ & $\begin{array}{l}\mathrm{R} \\
\mathrm{R} \\
\mathrm{R} \\
\mathrm{R} \\
\mathrm{R} \\
\mathrm{R} \\
\mathrm{R} \\
\mathrm{R} \\
\mathrm{R}\end{array}$ & & & $\begin{array}{l}R \\
R \\
R \\
R \\
F \\
R \\
F \\
R \\
F \\
\end{array}$ & $\begin{array}{l}\mathrm{F} \\
\mathrm{F} \\
\mathrm{F} \\
\mathrm{F} \\
\mathrm{C} \\
\mathrm{F} \\
\mathrm{A} \\
\mathrm{C}\end{array}$ & $\begin{array}{l}\mathrm{F} \\
\mathrm{F} \\
\mathrm{C} \\
\mathrm{F} \\
\mathrm{F} \\
\mathrm{F} \\
\mathrm{F} \\
\mathrm{F}\end{array}$ & \\
\hline $\begin{array}{l}9 \mathrm{H}-3,25-27 \\
9 \mathrm{H}, \mathrm{CC} \\
10 \mathrm{H}-3,25-27 \\
10 \mathrm{H}, \mathrm{CC} \\
11 \mathrm{H}-3,25-27 \\
11 \mathrm{H}, \mathrm{CC} \\
12 \mathrm{H}-3,25-27 \\
12 \mathrm{H}, \mathrm{CC} \\
13 \mathrm{H}-3,25-27 \\
13 \mathrm{H}, \mathrm{CC} \\
14 \mathrm{H}-3,25-27 \\
14 \mathrm{H}, \mathrm{CC} \\
15 \mathrm{H}-3,25-27 \\
15 \mathrm{H}, \mathrm{CC} \\
16 \mathrm{H}-3,25-27\end{array}$ & $\begin{array}{l}\mathrm{R} \\
\mathrm{R} \\
\mathrm{R} \\
\\
\mathrm{R} \\
\mathrm{F} \\
\mathrm{F}\end{array}$ & $\mathbf{R}$ & $\mathbf{R}$ & & $F$ & $\begin{array}{l}\mathrm{F} \\
\mathrm{R}\end{array}$ & $F$ & $\mathrm{~T}$ & $\begin{array}{l}\mathrm{R} \\
\mathrm{R} \\
\mathrm{R} \\
\mathrm{R} \\
\mathrm{R} \\
\mathrm{R} \\
\mathrm{R} \\
\mathrm{R}\end{array}$ & $\mathbf{R}$ & $\begin{array}{l}\mathrm{R} \\
\mathrm{R}\end{array}$ & & $\begin{array}{l}\mathrm{R} \\
\mathrm{R} \\
\mathrm{R} \\
\mathrm{R} \\
\mathrm{R} \\
\mathrm{R} \\
\mathrm{R} \\
\mathrm{R}\end{array}$ & $\mathbf{R}$ & $\begin{array}{l}\mathrm{R} \\
\mathrm{R} \\
\mathrm{R} \\
\mathrm{A}\end{array}$ & & & & $\begin{array}{l}\mathrm{R} \\
\mathrm{F} \\
\mathrm{F} \\
\mathrm{R}\end{array}$ & $\begin{array}{l}\mathrm{F} \\
\mathrm{F} \\
\mathrm{F} \\
\mathrm{F} \\
\mathrm{F} \\
\mathrm{R} \\
\mathrm{F} \\
\mathrm{F}\end{array}$ & $\begin{array}{c}R \\
R \\
R \\
R \\
R \\
R \\
R \\
\text { SR } \\
\text { R } \\
\text { R } \\
\text { R } \\
\text { R }\end{array}$ & $\mathbf{R}$ & $\begin{array}{l}\mathrm{F} \\
\mathrm{R} \\
\mathrm{R}\end{array}$ & $\begin{array}{l}\mathrm{F} \\
\mathrm{R} \\
\mathrm{F}\end{array}$ & $\begin{array}{l}\mathrm{R} \\
\mathrm{F} \\
\mathrm{R} \\
\mathrm{R} \\
\mathrm{R} \\
\mathrm{R} \\
\mathrm{F} \\
\mathrm{F} \\
\mathrm{F}\end{array}$ & $\begin{array}{l}\mathrm{F} \\
\mathrm{F} \\
\mathrm{F} \\
\mathrm{F} \\
\mathrm{F} \\
\mathrm{F} \\
\mathrm{F} \\
\mathrm{F} \\
\mathrm{F} \\
\mathrm{F} \\
\mathrm{F} \\
\mathrm{F} \\
\mathrm{R} \\
\mathrm{R}\end{array}$ & F \\
\hline
\end{tabular}

Figure 6. Stratigraphic ranges of selected planktonic foraminifers at Hole 628A.

species sensu stricto (Fig. 7). The top of the early Pliocene Globorotalia margaritae Zone (Globorotalia margaritae evoluta Subzone) was tentatively placed at the level of Sample 101-630A$6 \mathrm{H}-4,25-27 \mathrm{~cm}$, where $G$. sp. cf. $G$. margaritae margaritae has its LO (Fig. 7).

The first downhole occurrences of $G$. miocenica and $G$. exilis also are in this sample (Fig. 7). Forms falling within the strict definition of $G$. margaritae margaritae and $G$. margaritae evoluta have their first downhole occurrences in Section 101-630A$7 \mathrm{H}, \mathrm{CC}$ and clearly indicate the presence of the Globorotalia margaritae evoluta Subzone at this stratigraphic level (Fig. 7). Sediments occurring in this subzone extend down to the level of Sample 101-630A-11H-3, 25-27 cm, where the nominate taxon first appears (Fig. 7). The interval between Section 101-630A$11 \mathrm{H}, \mathrm{CC}$ and Sample 101-630-14H-3, 25-27 cm, occurs in the Globorotalia margaritae margaritae Subzone (Fig. 7).

Uppermost Miocene sediments (upper Neogloboquadrina humerosa Zone) are present in the interval between Sections 101$630 \mathrm{~A}-14 \mathrm{H}, \mathrm{CC}$ and $101-630 \mathrm{~A}-23 \mathrm{X}, \mathrm{CC}$, as indicated by the presence of the nominate taxon and Candeina nitida (Fig. 7). As at most Leg 101 sites, specimens of $N$. humerosa occur sporadically and in small numbers, possibly due to the poor preservation that characterizes most of the upper Miocene and younger section. Thus, the lowest downhole occurrence of this species in Sample 101-630A-17H-3, 25-27 cm, was not considered its first evolutionary appearance. Specimens of Globigerinoides extremus and Globorotalia plesiotumida occur down to the level of Sections 101-630A-25X, CC and 101-630-26X, CC, respectively (Fig. 7). Thus, the oldest sediments recovered at Hole 630A occur in the latest Miocene Neogloboquadrina humerosa Zone (Fig. 7).

\section{EXUMA SOUND TRANSECT-SITES 632, 633, AND 631}

A second slope transect was drilled to compare and to contrast the development of the "bypass" slope of Exuma Sound with the "accretionary" slope of Little Bahama Bank (Austin, Schlager, et al., 1986). Slope gradients in Exuma Sound are greater than those of Little Bahamas Bank $\left(10^{\circ}-12^{\circ}\right.$ vs. $\left.2^{\circ}-3^{\circ}\right)$. In addition, relief is also greater in Exuma Sound ( $1600 \mathrm{~m}$ vs. $800-900 \mathrm{~m}$ ). As a result of these factors, bypassing of the slope by turbidity currents was expected to be more complete in Exuma Sound, so sediments there would be predominantly periplatform oozes, especially toward the proximal end of the transect (Site 631) (Austin, Schlager, et al., 1986).

Another objective of the Exuma Sound transect was to compare the response to sea-level changes of the protected environment of Exuma Sound with that of the open-ocean setting at Little Bahama Bank (Austin, Schlager, et al., 1986).

\section{Site 632}

Two holes drilled at Site 632 (water depth of $1996 \mathrm{~m}$ ) form the basinward end of the Exuma Sound transect (Fig. 1). At Hole $632 \mathrm{~A}, 16$ cores were retrieved using the HPC and XCB; penetration reached to $141.0 \mathrm{mbsf}$. About $83 \mathrm{~m}$ of Neogene sediments (56\% recovery) was recovered. Recovery was much higher in the upper eight cores (average 86\%) than in the lower eight (average 26\%) (Austin, Schlager, et al., 1986),

Hole 632B was washed to 120.7 mbsf and then rotary cored to $283.2 \mathrm{mbsf}$, for a cored section of $162.6 \mathrm{~m}$. Seventeen cores contained $34.8 \mathrm{~m}$ of sediment ( $21 \%$ recovery). 


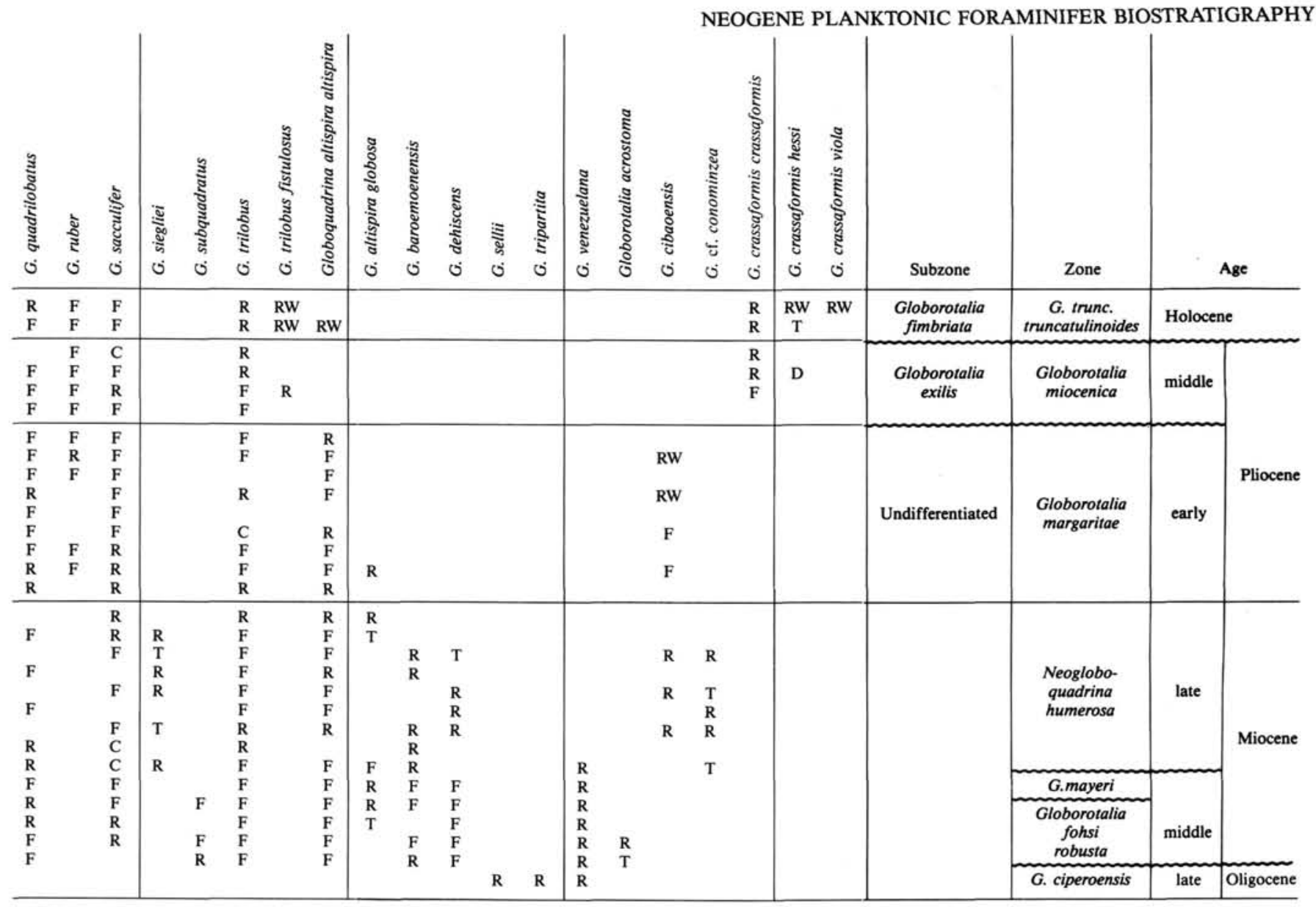

Figure 6 (continued).

Pleistocene (Globorotalia truncatulinoides truncatulinoides Zone) sediments are present in Sample 101-632A-1H-3, 25-27 $\mathrm{cm}$, and extend through Section 101-632A-5H, CC. The early Pleistocene Globorotalia crassaformis hessi Subzone was observed in Samples 101-632A-1H-3, 25-27 cm, through 101-632A$2 \mathrm{H}, \mathrm{CC}$, based on the presence of the nominate taxon without Globigerina calida calida (Parker) (Fig. 8). The absence of Globorotalia crassaformis hessi from Samples 101-632A-3H-3, 25$27 \mathrm{~cm}$, through 101-632A-5H, CC indicates that this interval occurs in the earliest Pleistocene Globorotalia crassaformis viola Subzone (Fig. 8). Calcareous nannofossils examined from Sample $101-632 \mathrm{~A}-6 \mathrm{H}-1,10-12 \mathrm{~cm}$, indicate that this level is also of Pleistocene age (Watkins and Verbeek, this volume). The Pleistocene sediments at Site 632 contain abundant shallow-water, platform-derived material, which was interpreted as downhole contamination. However, it may be that the entire section is younger than is indicated by the (reworked?) early Pleistocene zonal marker species.

An unconformity separates the early Pleistocene from the middle Pliocene at Site 632 (Fig. 8). Sample 101-632A-6H-3, $25-27 \mathrm{~cm}$, contains Globorotalia miocenica without G. margaritae, thus identifying the middle Pliocene Globorotalia miocenica Zone (Fig. 8). The absence of Globigerinoides trilobus fistulosus in Sample 101-632A-6H-3, 25-27 cm, indicates the presence of the Globorotalia exilis Subzone at this level (Fig. 8). According to Bolli and Saunders (1985), the range of Globoquadrina altispira altispira does not extend into the Globorotalia exilis Subzone. The absence of this species in Section 101$632 \mathrm{~A}-6 \mathrm{H}, \mathrm{CC}$ further supports the subzone assigned here (Fig. 8).

The early Pliocene Globorotalia margaritae Zone was first encountered in Section 101-632A-6H, CC, where the co-occur- rence of $G$. miocenica with $G$. margaritae indicates the presence of the uppermost part of the Globorotalia margaritae Zone (upper Globorotalia margaritae evoluta Subzone) (Fig. 8). Lower Pliocene sediments continue downward through Section 101632A-13X, CC (Core 101-632A-11X failed to extrude) (Fig. 8). Preservation in this interval was generally poor, which probably accounts for the absence or scarcity of $G$. margaritae in several samples (Fig. 8). In addition, most foraminifers in this interval were covered with a heavy overgrowth that could not always be removed by ultrasonic cleaning. Thus, the subspecies of $G$. margaritae could not be differentiated consistently enough to subdivide the Globorotalia margaritae Zone in Hole 632A (Fig. 8).

The Miocene/Pliocene boundary at Site 632 was difficult to place. Calcareous nannofossils suggest a late Miocene age for Sample 101-632A-12X-5, $80 \mathrm{~cm}$ (Watkins and Verbeek, this volume). Specimens of the Pliocene marker species Globorotalia margaritae occur sporadically down to Section 101-632A-13X, $\mathrm{CC}$, which suggests that this interval correlates with the early Pliocene Globorotalia margaritae Zone (Fig. 8). However, specimens of $G$. juanai Bermudez and Bolli are present in much of the lower part of the Globorotalia margaritae Zone up to Sample 101-632A-9H-3, 25-27 cm (Fig. 8). According to Kennett and Srinivasan (1983) and Bolli and Saunders (1985), the range of $G$. juanai is restricted to the late Miocene and does not overlap that of $G$. margaritae. This suggests that either specimens of G. juanai were reworked into Pliocene sediments or that the specimens of $G$. margaritae below Section 101-632-8H, CC were caved. In the latter case, the Miocene/Pliocene boundary would be placed between Section 101-632A-8H, CC and Sample 101$632 \mathrm{~A}-9 \mathrm{H}-3,25-27 \mathrm{~cm}$. This is approximately 20 to $23 \mathrm{~m}$ above the boundary, as identified using calcareous nannofossils, which 


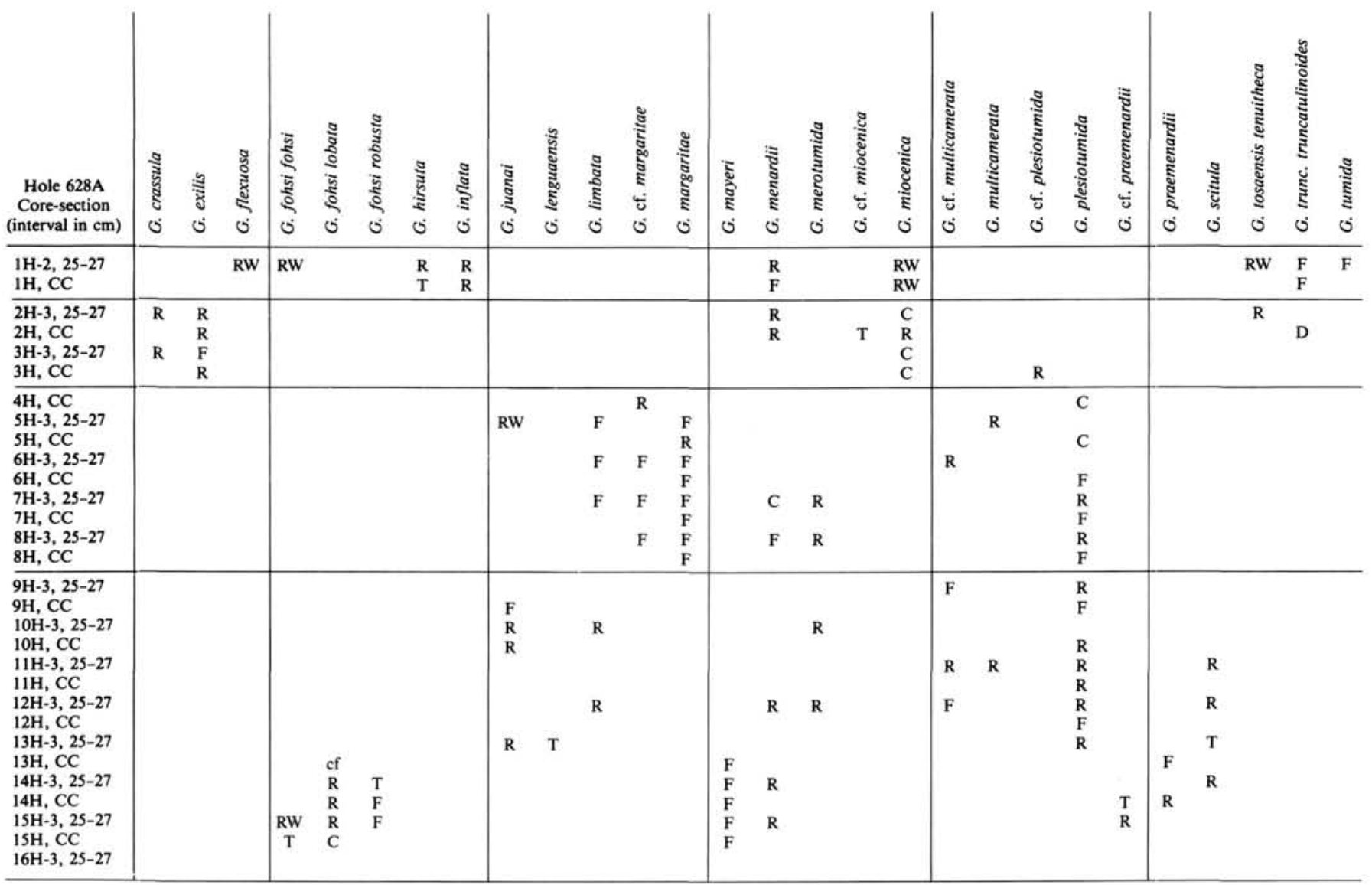

Figure 6 (continued).

does not seem likely. The specimens of $G$. juanai are apparently reworked, which means the top of the Miocene lies below the level of Section 101-632A-13X, CC (Fig. 8).

Only core-catcher samples that contain limestone were recovered from Cores 101-632A-14X, -15X, and -16X. Preservation was poor, and no zonal marker species were identified (Fig. 8). However, if in-situ, the presence of Pulleniatina primalis Banner and Blow in Section 101-632A-14X, CC suggests an early Pliocene age, thus supporting the assignment of an early Pliocene age to the sediments in Cores 101-632A-12X and 101-632A-13X (Fig. 8).

At Hole 632B, the combination of low core recovery and poor preservation made it difficult and inexact to determine ages. In most cases, only a few foraminifers could be identified to the species level. However, in core-catcher samples from Cores 101-632B-8R, -10R, -12R, and -13R, the absence of G. margaritae margaritae and the presence of Globigerinoides extremus, Sphaeroidinellopsis paenedehiscens, and Globorotalia sp. cf. G. plesiotumida suggest assignment to the latest Miocene Neogloboquadrina humerosa Zone. Thus, the Miocene/Pliocene boundary apparently lies between Sections 101-632A-14X, CC and 101-632B-8R, CC.

\section{Site 633}

Site 633 is located a few kilometers basinward of the toe-ofslope at a water depth of $1681 \mathrm{~m}$ (Fig. 1). The only hole drilled at this site serves as a tie between the more basinward Site 632 and Site 631 , located on the upper slope. Twenty-four cores were recovered using the HPC and XCB while penetrating to a depth of $227.3 \mathrm{mbsf}$. Total core recovered was $110.8 \mathrm{~m}$, or $48.7 \%$ of the cored interval.
Pleistocene sediments are present in Sample 101-633A-1H-3, $25-27 \mathrm{~cm}$, and continue down to Sample 101-633A-5H-3, 25-27 $\mathrm{cm}$ (Fig. 9). Calcareous nannofossils indicate a latest Pleistocene to Holocene age (CN15) for Sample 101-633A-1H-1, $1 \mathrm{~cm}$ (Watkins and Verbeek, this volume). Planktonic foraminifers indicate a middle Pleistocene age (Globorotalia crassaformis hessi Subzone) for the interval from Sample 101-633A-1H-3, 25-27 cm, through Sample 101-633A-2H-3, 25-27 cm (Fig. 9). Specimens of the nominate taxon are rare in this interval but do not appear to be reworked (Fig. 9). Based on the absence of $G$. crassaformis hessi, the interval from Section 101-633A-2H, CC through Sample $101-633 \mathrm{~A}-5 \mathrm{H}-3,25-27 \mathrm{~cm}$, was assigned to the early Pleistocene Globorotalia crassaformis viola Subzone (Fig. 9).

The late Pliocene Globorotalia tosaensis tosaensis Zone appears to be absent in Hole $633 \mathrm{~A}$, which suggests the presence of an unconformity (Fig. 9). Sample 101-633A-5H-5, 129-131 cm, contains sediments that correlate with the middle Pliocene Globorotalia miocenica Zone (Globorotalia exilis Subzone) (Fig. 9). This zonal assignment is based on the absence of $G$. tosaensis tosaensis Takayanagi and Saito and the presence of $G$. crassaformis viola (Fig. 9). According to Bolli and Saunders (1985), the absence of $G$. tosaensis tosaensis suggests a middle Pliocene or older age, while the presence of $G$. crassaformis viola indicates an age no greater than late middle Pliocene (Globorotalia exilis Subzone). Further support for this zonal assignment is the absence of $G$. margaritae s.l. and the presence of a juvenile, keeled globorotaliid, referred to Globorotalia sp. cf. G. miocenica, which has a flat spiral side and six chambers that increase irregularly in size (Fig. 9).

The next two samples examined (101-633A-5H-6, 18-20 cm, and $101-633 \mathrm{~A}-5 \mathrm{H}, \mathrm{CC}$ ) contain $G$. margaritae margaritae and 


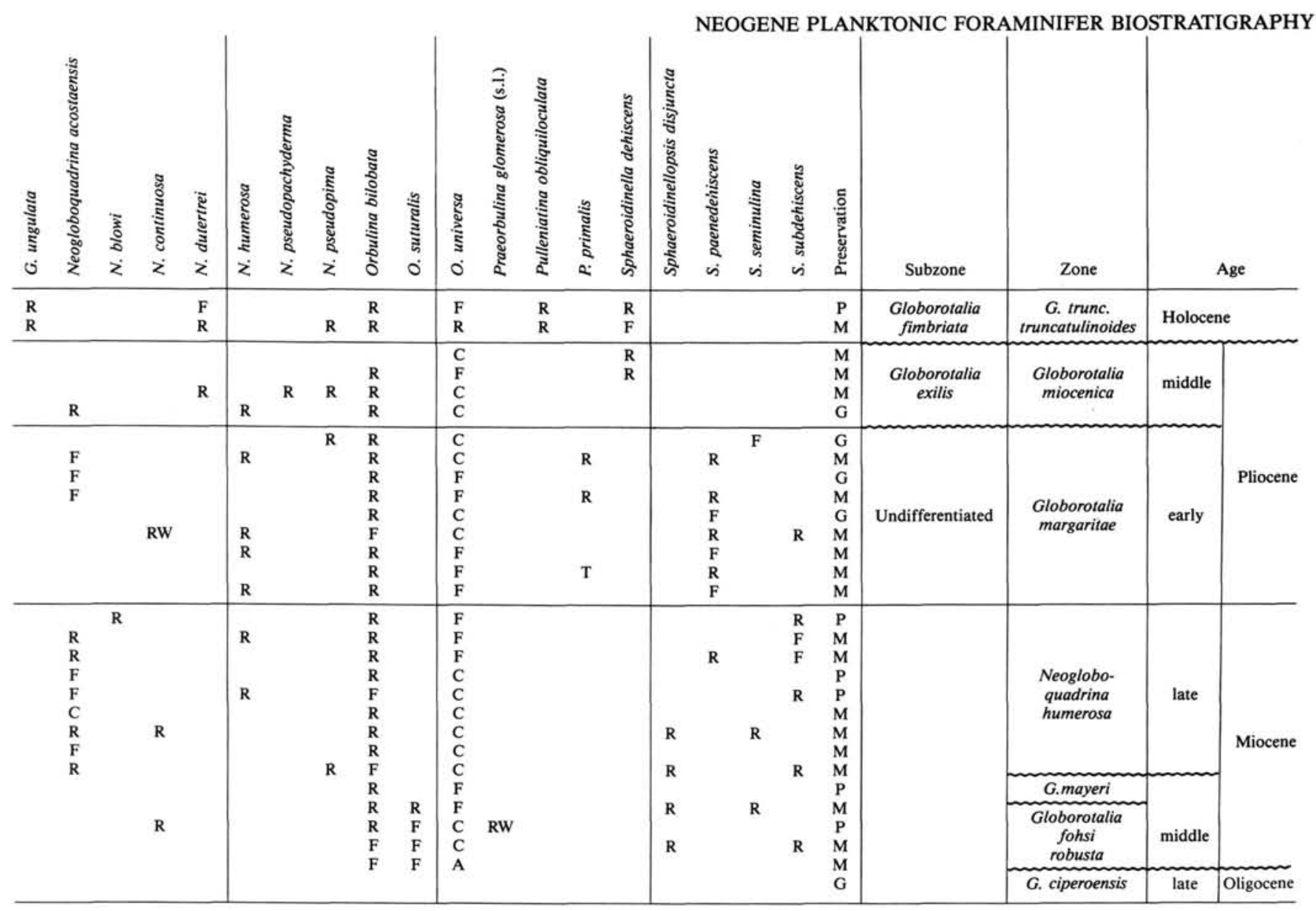

Figure 6 (continued).

G. margaritae evoluta, thus identifying the late early Pliocene Globorotalia margaritae evoluta Subzone (Fig. 9). This suggests the presence of an unconformity in which the early middle Pliocene Globigerinoides trilobus fistulosus Subzone and the uppermost Globorotalia margaritae evoluta Subzone are missing (Fig. 9). I could not assign age subzones for the interval from Sample 101-633A-6H-3, 25-27 cm, through Section 101-633A$14 \mathrm{X}, \mathrm{CC}$ because of the poor to very poor preservation of the planktonic foraminifers (Fig. 9).

The top of the Globorotalia margaritae margaritae Subzone is at or above the level of Section 101-633A-15X, CC, based on the highest occurrence of $G$. margaritae primitiva Cita (Fig. 9). The actual boundary may be higher, but could not be recognized because of the poor preservation discussed above.

The deepest sample containing identifiable planktonic foraminifers (101-633A-23X-1, 62-64 cm) has a fauna that includes the nominate taxon of the Globorotalia margaritae margaritae Subzone as well as $G$. margaritae primitiva and $G$. crassaformis s.l., suggesting a Pliocene age for this level (Fig. 9). Section 101633A-23X, CC did not contain identifiable planktonic foraminifers. The assignment of a Pliocene age to sediments in the lower part of Hole 633A differs from the shipboard assignment of a late Miocene age for sediments from Section 101-633A-15X, CC and below (Austin, Schlager, et al., 1986). This is due to more intensive shore-based study of the core-catcher samples than was possible while at sea, as well as the examination of an additional sample (101-633A-23X-1, 62-64 cm). However, calcareous nannofossils still support a late Miocene age (CN9b) for this interval (Watkins and Verbeek, this volume). It may be that the nannofossils are reworked or that the planktonic foraminifers are downhole contaminants. Unfortunately, neither possi- bility can be excluded because reworking of nannofossils is common, and occurrences of G. margaritae in the lower part of the hole are sporadic, possibly owing in part to downhole caving. Thus, the age of the sediments at the bottom of Hole $633 \mathrm{~A}$ can be constrained to an age of latest Miocene to earliest Pliocene.

\section{Site 631}

One hole was drilled at Site 631 , at the shallow end of the Exuma Sound transect (Fig. 1). This hole was drilled at a water depth of $1081 \mathrm{~m}$ using the HPC and XCB and penetrated to 244.3 mbsf (Austin, Schlager, et al., 1986). Drilling of 25 cores retrieved a total of $159 \mathrm{~m}$ of sediment ( $65 \%$ recovery).

Preservation of planktonic foraminifers at Hole 631A was the poorest of any Neogene-Holocene section drilled during Leg 101. Only the sediments in Core 101-631A-1H were well preserved (Fig. 10). Preservation was poor in almost every sample below this core (Fig. 10). Typically, the sediments were chalky and/or contained foraminifers that were so heavily overgrown that identification to the generic level was often difficult. This was especially common below Core 101-631A-12X. In most cases, identification to a species level was only possible after individual specimens were placed in an ultrasonic bath for several seconds to dislodge these overgrowths.

The very poor preservation at Hole $631 \mathrm{~A}$ resulted in uncertainties regarding the placement of the early/middle Pliocene boundary and the age of the sediments below Core 101-631A-5H. In the case of the former, the boundary was tentatively placed on the basis of the occurrences of secondary zonal marker species. In the latter case, an age of early Pliocene was assigned, based on the fauna in Section 101-631A-25X, CC even though no unequivocal age assignment could be made in Cores 101- 


\begin{tabular}{|c|c|c|c|c|c|c|c|c|c|c|c|c|c|c|c|c|c|c|c|c|c|c|c|c|c|}
\hline $\begin{array}{c}\text { Hole 630A } \\
\text { Core-section } \\
\text { (interval in cm) }\end{array}$ & 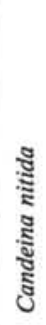 & 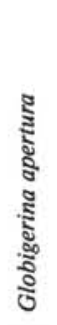 & 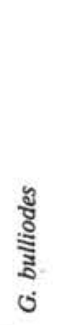 & 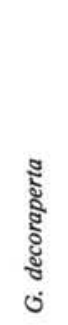 & $\begin{array}{l}\overline{\mathrm{s}} \\
\text { ธิ } \\
0\end{array}$ & 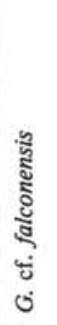 & $\underbrace{\frac{n}{5}}_{0}$ & 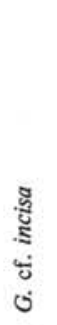 & 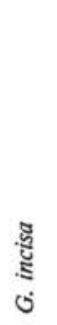 & 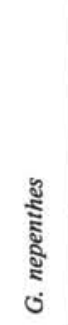 & 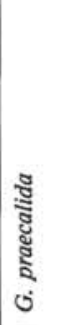 & 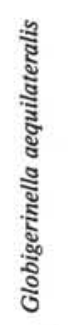 & $\begin{array}{l}\text { ฐँ๊ } \\
8 \\
ن\end{array}$ & 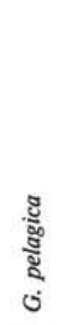 & 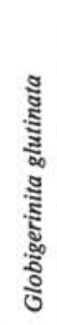 & 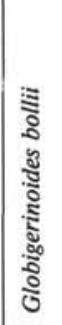 & 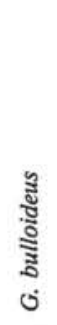 & 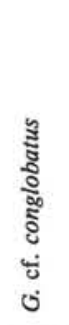 & 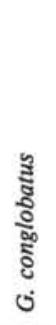 & 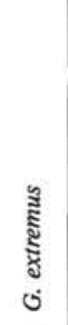 & 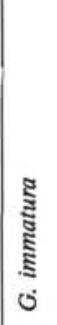 & $\begin{array}{l}\text { : } \\
0 \\
0 \\
0\end{array}$ & 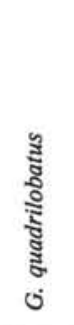 & है & 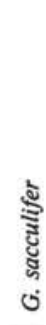 \\
\hline $1 \mathrm{H}-3,25-27$ & $\mathrm{R}$ & & & RW & & & $\mathbf{R}$ & & & RW & & $\mathbf{R}$ & & & $\mathbf{R}$ & & & $\mathrm{F}$ & & RW & $\mathrm{F}$ & RW & $\mathbf{R}$ & & F \\
\hline $\begin{array}{l}1 \mathrm{H}-4,25-27 \\
1 \mathrm{H}, \mathrm{CC}\end{array}$ & $\begin{array}{l}\mathrm{R} \\
\mathrm{R} \\
\end{array}$ & & & & & & & & & & $\begin{array}{l}\mathbf{R} \\
\mathrm{R} \\
\end{array}$ & $\begin{array}{l}\mathrm{R} \\
\mathrm{F}\end{array}$ & $\begin{array}{l}\mathrm{F} \\
\mathrm{R}\end{array}$ & & $\begin{array}{l}\mathrm{R} \\
\mathrm{R} \\
\end{array}$ & & & & $\begin{array}{l}\mathrm{R} \\
\mathrm{R} \\
\end{array}$ & & $\begin{array}{l}\mathrm{F} \\
\mathrm{F} \\
\end{array}$ & & $\mathbf{R}$ & $\begin{array}{l}\mathrm{F} \\
\mathrm{C}\end{array}$ & $\begin{array}{l}\mathrm{F} \\
\mathrm{F}\end{array}$ \\
\hline $\begin{array}{l}2 \mathrm{H}-4,25-27 \\
2 \mathrm{H}, \mathrm{CC}\end{array}$ & $\mathrm{R}$ & & & $\mathrm{T}$ & & & & & $\begin{array}{l}\mathrm{R} \\
\mathrm{R}\end{array}$ & & R & $\begin{array}{l}\mathrm{C} \\
\mathrm{F}\end{array}$ & $T$ & $\mathbf{R}$ & $\begin{array}{l}\mathbf{R} \\
\mathbf{R}\end{array}$ & & & $\mathbf{R}$ & $\mathbf{R}$ & & $\begin{array}{l}\text { R } \\
\text { F } \\
\end{array}$ & & $\begin{array}{l}\mathrm{T} \\
\mathrm{R}\end{array}$ & $\begin{array}{l}\mathrm{C} \\
\mathrm{F}\end{array}$ & $\begin{array}{l}\text { F } \\
\text { C }\end{array}$ \\
\hline $\begin{array}{l}3 \mathrm{H}-4,25-27 \\
3 \mathrm{H}, \mathrm{CC} \\
4 \mathrm{H}-3,25-27 \\
4 \mathrm{H}, \mathrm{CC} \\
5 \mathrm{H}-3,25-27 \\
5 \mathrm{H}, \mathrm{CC} \\
\end{array}$ & $\mathbf{R}$ & & & $\begin{array}{l}\mathrm{R} \\
\mathrm{R} \\
\mathrm{R} \\
\mathrm{R}\end{array}$ & & & & $\mathbf{R}$ & $\begin{array}{l}\mathrm{R} \\
\mathrm{R} \\
\mathrm{R} \\
\mathrm{R} \\
\mathrm{R} \\
\end{array}$ & & $\begin{array}{l}\mathrm{R} \\
\mathrm{R} \\
\mathrm{R} \\
\mathrm{R}\end{array}$ & $\begin{array}{l}\mathrm{F} \\
\mathrm{F} \\
\mathrm{R} \\
\mathrm{F} \\
\mathrm{F} \\
\mathrm{R} \\
\end{array}$ & $\begin{array}{l}\mathrm{R} \\
\mathrm{R} \\
\mathrm{R} \\
\end{array}$ & $\begin{array}{l}\mathrm{R} \\
\mathrm{R}\end{array}$ & $\begin{array}{l}\mathrm{R} \\
\mathrm{R} \\
\mathrm{R} \\
\mathrm{R} \\
\mathrm{R} \\
\mathrm{R}\end{array}$ & & & & $\begin{array}{l}\mathrm{R} \\
\mathrm{R} \\
\mathrm{R} \\
\mathrm{R} \\
\mathrm{R} \\
\mathrm{R} \\
\end{array}$ & $\mathbf{R}$ & $\begin{array}{l}\mathrm{F} \\
\mathrm{F} \\
\mathrm{R} \\
\mathrm{F} \\
\mathrm{F} \\
\mathrm{F} \\
\end{array}$ & $\mathbf{R}$ & $\begin{array}{l}\mathrm{R} \\
\mathrm{R} \\
\mathrm{R} \\
\mathrm{R} \\
\mathrm{R} \\
\mathrm{R} \\
\end{array}$ & $\begin{array}{l}\mathrm{F} \\
\mathrm{F} \\
\mathrm{R} \\
\mathrm{F} \\
\mathrm{F} \\
\mathrm{F} \\
\end{array}$ & $\begin{array}{l}\mathrm{C} \\
\mathrm{C} \\
\mathrm{C} \\
\mathrm{F} \\
\mathrm{F} \\
\mathrm{F}\end{array}$ \\
\hline $\begin{array}{l}6 \mathrm{H}-4,25-27 \\
6 \mathrm{H}, \mathrm{CC} \\
7 \mathrm{H}-4,25-27 \\
7 \mathrm{H}, \mathrm{CC} \\
8 \mathrm{H}-3,25-27 \\
8 \mathrm{H}, \mathrm{CC} \\
9 \mathrm{H}-3,25-27 \\
9 \mathrm{H}, \mathrm{CC} \\
10 \mathrm{H}-3,25-27 \\
10 \mathrm{H}, \mathrm{CC} \\
11 \mathrm{H}-3,25-27 \\
\end{array}$ & $\begin{array}{l}\mathrm{T} \\
\mathrm{T} \\
\mathrm{R} \\
\mathrm{R}\end{array}$ & & $\begin{array}{l}R \\
R \\
R \\
R\end{array}$ & $\begin{array}{l}\mathrm{R} \\
\mathrm{F} \\
\mathrm{R}\end{array}$ & & $\begin{array}{l}\mathrm{R} \\
\mathrm{R} \\
\mathrm{R}\end{array}$ & $\begin{array}{l}\mathrm{R} \\
\mathrm{R}\end{array}$ & $\begin{array}{l}\mathrm{F} \\
\mathrm{R} \\
\mathrm{R}\end{array}$ & $\begin{array}{l}\mathrm{R} \\
\mathrm{R}\end{array}$ & $\mathbf{R}$ & $\begin{array}{l}\mathbf{R} \\
\mathbf{R}\end{array}$ & $\begin{array}{l}\mathrm{R} \\
\mathrm{R} \\
\mathrm{R} \\
\mathrm{P} \\
\mathrm{F} \\
\mathrm{F} \\
\mathrm{F} \\
\mathrm{F} \\
\mathrm{R} \\
\mathrm{R}\end{array}$ & $\begin{array}{l}\mathrm{R} \\
\mathrm{R} \\
\mathrm{R} \\
\\
\\
\mathrm{R} \\
\mathrm{R} \\
\mathrm{R}\end{array}$ & & $\begin{array}{l}\mathrm{R} \\
\mathrm{R} \\
\mathrm{F} \\
\mathrm{F} \\
\mathrm{P} \\
\mathrm{R} \\
\mathrm{R} \\
\mathrm{R} \\
\mathrm{F} \\
\mathrm{F} \\
\mathrm{F}\end{array}$ & $\mathrm{T}$ & & $\mathbf{R}$ & $\begin{array}{l}\mathrm{F} \\
\mathrm{F} \\
\mathrm{R} \\
\mathrm{P} \\
\mathrm{F} \\
\mathrm{R} \\
\mathrm{R} \\
\mathrm{R} \\
\mathrm{R} \\
\mathrm{R} \\
\end{array}$ & $\begin{array}{l}\mathrm{F} \\
\\
\mathrm{F} \\
\mathrm{F} \\
\mathrm{R} \\
\mathrm{R} \\
\mathrm{F} \\
\mathrm{F} \\
\end{array}$ & $\begin{array}{l}\mathrm{F} \\
\mathrm{F} \\
\mathrm{F} \\
\mathrm{F} \\
\mathrm{P} \\
\mathrm{F} \\
\mathrm{F} \\
\mathrm{F} \\
\mathrm{F} \\
\mathrm{F} \\
\mathrm{F} \\
\end{array}$ & $\begin{array}{l}\mathrm{R} \\
\mathrm{R} \\
\mathrm{R} \\
\mathrm{F} \\
\mathrm{R}\end{array}$ & $\begin{array}{l}\mathrm{R} \\
\mathrm{R} \\
\mathrm{R} \\
\mathrm{R} \\
\mathrm{F} \\
\mathrm{F} \\
\mathrm{F} \\
\mathrm{T} \\
\mathrm{R} \\
\mathrm{R} \\
\end{array}$ & $\begin{array}{l}\mathrm{F} \\
\mathrm{F} \\
\mathrm{F} \\
\mathrm{R} \\
\mathrm{F} \\
\mathrm{F} \\
\mathrm{R}\end{array}$ & $\begin{array}{l}\mathrm{F} \\
\mathrm{R} \\
\mathrm{R} \\
\mathrm{F} \\
\\
\mathrm{F} \\
\mathrm{R} \\
\mathrm{F} \\
\mathrm{R} \\
\mathrm{R} \\
\mathrm{R}\end{array}$ \\
\hline $\begin{array}{l}11 \mathrm{H}, \mathrm{CC} \\
12 \mathrm{H}-3,25-27 \\
12 \mathrm{H}, \mathrm{CC} \\
13 \mathrm{H}-3,25-27 \\
13 \mathrm{H}, \mathrm{CC} \\
14 \mathrm{H}-3,25-27 \\
\end{array}$ & $\begin{array}{l}\mathrm{T} \\
\mathrm{R} \\
\mathrm{R} \\
\mathrm{R} \\
\end{array}$ & $\begin{array}{l}T \\
T\end{array}$ & $\mathrm{~T}$ & $\begin{array}{l}\mathrm{R} \\
\mathrm{F} \\
\mathrm{F} \\
\mathrm{R} \\
\mathrm{R} \\
\mathrm{R}\end{array}$ & & $\mathbf{R}$ & & $\mathrm{T}$ & & $\begin{array}{l}\mathbf{R} \\
\mathbf{R} \\
\mathrm{R} \\
\mathrm{R} \\
\mathbf{R} \\
\mathrm{R}\end{array}$ & & $\begin{array}{l}\mathrm{R} \\
\mathrm{R} \\
\mathrm{R} \\
\mathrm{R} \\
\mathrm{R} \\
\mathrm{F}\end{array}$ & $\begin{array}{l}\mathrm{R} \\
\mathrm{R} \\
\mathrm{R} \\
\mathrm{F} \\
\end{array}$ & & $\begin{array}{l}\mathrm{R} \\
\mathrm{F} \\
\mathrm{R} \\
\mathrm{R} \\
\mathrm{R} \\
\mathrm{R}\end{array}$ & & $\mathbf{R}$ & & $\begin{array}{l}\mathrm{R} \\
\mathrm{R} \\
\mathrm{R} \\
\mathrm{F} \\
\mathrm{R} \\
\mathrm{R}\end{array}$ & $\begin{array}{l}\text { F } \\
C \\
F \\
F \\
F \\
F\end{array}$ & $\begin{array}{l}\mathrm{F} \\
\mathrm{F} \\
\mathrm{F} \\
\mathrm{F} \\
\mathrm{F} \\
\mathrm{F}\end{array}$ & $\begin{array}{l}\text { R } \\
\text { F } \\
\text { F } \\
\text { F } \\
\text { F } \\
\text { F }\end{array}$ & $\begin{array}{l}\mathrm{F} \\
\mathrm{R} \\
\mathrm{R} \\
\mathrm{R} \\
\mathrm{R} \\
\mathrm{F}\end{array}$ & & $\begin{array}{l}\mathrm{F} \\
\mathrm{R} \\
\mathrm{F} \\
\mathrm{F} \\
\mathrm{F} \\
\mathrm{F}\end{array}$ \\
\hline $\begin{array}{l}14 \mathrm{H}, \mathrm{CC} \\
15 \mathrm{H}, \mathrm{CC} \\
16 \mathrm{H}-3,25-27 \\
16 \mathrm{H}, \mathrm{CC} \\
17 \mathrm{H}-3,25-27 \\
17 \mathrm{H}, \mathrm{CC} \\
18 \mathrm{H}-3,25-27 \\
18 \mathrm{H}, \mathrm{CC} \\
19 \mathrm{X}-3,25-27 \\
19 \mathrm{X}, \mathrm{CC} \\
20 \mathrm{X}-3,25-27 \\
20 \mathrm{X}, \mathrm{CC} \\
21 \mathrm{X}-3,25-27 \\
21 \mathrm{X}, \mathrm{CC} \\
22 \mathrm{X}, \mathrm{CC} \\
23 \mathrm{X}-3,25-27 \\
23 \mathrm{X}, \mathrm{CC} \\
24 \mathrm{X}-3,25-27 \\
24 \mathrm{X}, \mathrm{CC} \\
25 \mathrm{X}, \mathrm{CC} \\
26 \mathrm{X}-3,25-27 \\
26 \mathrm{X}, \mathrm{CC}\end{array}$ & $\begin{array}{l}\mathrm{T} \\
\mathrm{R} \\
\mathrm{R}\end{array}$ & & $\mathbf{R}$ & $\begin{array}{l}R \\
R \\
R \\
R \\
R \\
R \\
R \\
R \\
R \\
R\end{array}$ & RW & R & & & & $\begin{array}{l}\text { R } \\
R \\
R \\
R \\
\text { F } \\
F \\
R \\
F \\
T \\
R \\
R \\
R \\
T \\
T \\
F \\
F \\
R \\
F \\
T \\
R \\
R \\
F \\
C\end{array}$ & & $\begin{array}{l}\mathrm{R} \\
\mathrm{R} \\
\mathrm{F} \\
\mathrm{F} \\
\mathrm{R} \\
\mathrm{F} \\
\mathrm{R} \\
\mathrm{R} \\
\mathrm{R} \\
\mathrm{R} \\
\mathrm{R} \\
\mathrm{R} \\
\mathrm{R} \\
\mathrm{R}\end{array}$ & $\begin{array}{l}\mathrm{R} \\
\mathrm{F} \\
\mathrm{R} \\
\\
\mathrm{R} \\
\mathrm{R} \\
\mathrm{R} \\
\mathrm{R} \\
\mathrm{R} \\
\mathrm{R}\end{array}$ & cf & $\begin{array}{l}\mathrm{R} \\
\mathrm{R} \\
\mathrm{R} \\
\mathrm{R} \\
\mathrm{R} \\
\mathrm{R} \\
\mathrm{R} \\
\mathrm{R} \\
\mathrm{R} \\
\mathrm{R} \\
\mathrm{R} \\
\mathrm{R} \\
\mathrm{R} \\
\mathrm{R} \\
\mathrm{R} \\
\mathrm{R} \\
\mathrm{R} \\
\mathrm{R} \\
\mathrm{R}\end{array}$ & $\begin{array}{l}\mathrm{T} \\
\mathrm{T} \\
\mathrm{R}\end{array}$ & $\mathrm{T}$ & $\mathbf{R}$ & $\begin{array}{l}\mathrm{T} \\
\mathrm{T} \\
\mathrm{T} \\
\mathrm{R} \\
\mathrm{R} \\
\mathrm{R} \\
\mathrm{R} \\
\mathrm{R} \\
\mathrm{R} \\
\mathrm{R} \\
\mathrm{R} \\
\mathrm{R} \\
\mathrm{R} \\
\mathrm{R}\end{array}$ & $\begin{array}{l}\mathrm{F} \\
\mathrm{C} \\
\mathrm{F} \\
\mathrm{F} \\
\mathrm{F} \\
\mathrm{F} \\
\mathrm{F} \\
\mathrm{F} \\
\mathrm{F} \\
\mathrm{F} \\
\mathrm{F} \\
\mathrm{R} \\
\mathrm{R} \\
\mathrm{F} \\
\mathrm{F} \\
\mathrm{F} \\
\mathrm{F} \\
\mathrm{R} \\
\mathrm{R} \\
\mathrm{F}\end{array}$ & \begin{tabular}{|l}
$\mathrm{F}$ \\
$\mathrm{F}$ \\
$\mathrm{F}$ \\
$\mathrm{F}$ \\
$\mathrm{F}$ \\
$\mathrm{F}$ \\
$\mathrm{F}$ \\
$\mathrm{F}$ \\
$\mathrm{F}$ \\
$\mathrm{F}$ \\
$\mathrm{F}$ \\
$\mathrm{C}$ \\
$\mathrm{C}$ \\
$\mathrm{F}$ \\
$\mathrm{F}$ \\
$\mathrm{R}$ \\
$\mathrm{F}$ \\
$\mathrm{F}$ \\
$\mathrm{F}$ \\
$\mathrm{F}$ \\
$\mathrm{F}$ \\
$\mathrm{F}$
\end{tabular} & $\begin{array}{l}\text { F } \\
R \\
F \\
F \\
F \\
F \\
F \\
F \\
F \\
F \\
F \\
F \\
F \\
F \\
F \\
F \\
F \\
F \\
F \\
F \\
R \\
F\end{array}$ & $\begin{array}{l}R \\
R \\
F \\
F \\
R \\
F \\
F \\
F \\
F \\
F\end{array}$ & $\mathrm{~T}$ & $\begin{array}{l}\mathrm{F} \\
\mathrm{F} \\
\mathrm{R} \\
\mathrm{R} \\
\mathrm{F} \\
\mathrm{R} \\
\mathrm{R} \\
\mathrm{R} \\
\mathrm{R} \\
\mathrm{R} \\
\mathrm{C} \\
\mathrm{C} \\
\mathrm{C} \\
\mathrm{F} \\
\mathrm{F} \\
\mathrm{F} \\
\mathrm{F} \\
\mathrm{R} \\
\mathrm{R} \\
\mathrm{R} \\
\mathrm{F} \\
\mathrm{R}\end{array}$ \\
\hline
\end{tabular}

Figure 7. Stratigraphic ranges of selected planktonic foraminifers at Hole 630A.

631A-15X through 101-631A-24X. These problems are discussed in more detail next.

The sediments of Cores 101-631A-1H and 101-631A-2H are in the Globorotalia truncatulinoides truncatulinoides Zone, based on the presence of the nominate taxon (Fig. 10). Sample 101-631A-1H-3, 25-27 cm, is Holocene in age (Globorotalia fimbriata Subzone), as indicated by the presence of $G$. ungulata (Fig. 10). Sections 101-631A-1H, CC through 101-631A-2H, CC contain G. crassaformis hessi without Globigerina calida calida, which indicates that this interval is in the mid-Pleistocene Globorotalia crassaformis hessi Subzone (Fig. 10). This suggests that the late Pleistocene Globigerina bermudezi Subzone and Globigerina calida calida Subzone are missing (Figs. 2 and 10).

As at Sites 632 and 633, an unconformity appears to mark the contact between the Pleistocene and the Pliocene, with the late Pliocene Globorotalia tosaensis tosaensis Zone missing (Fig. 10). However, unlike the section at Sites 632 and 633, in Hole 631A the early Pleistocene Globorotalia crassaformis viola Sub- 


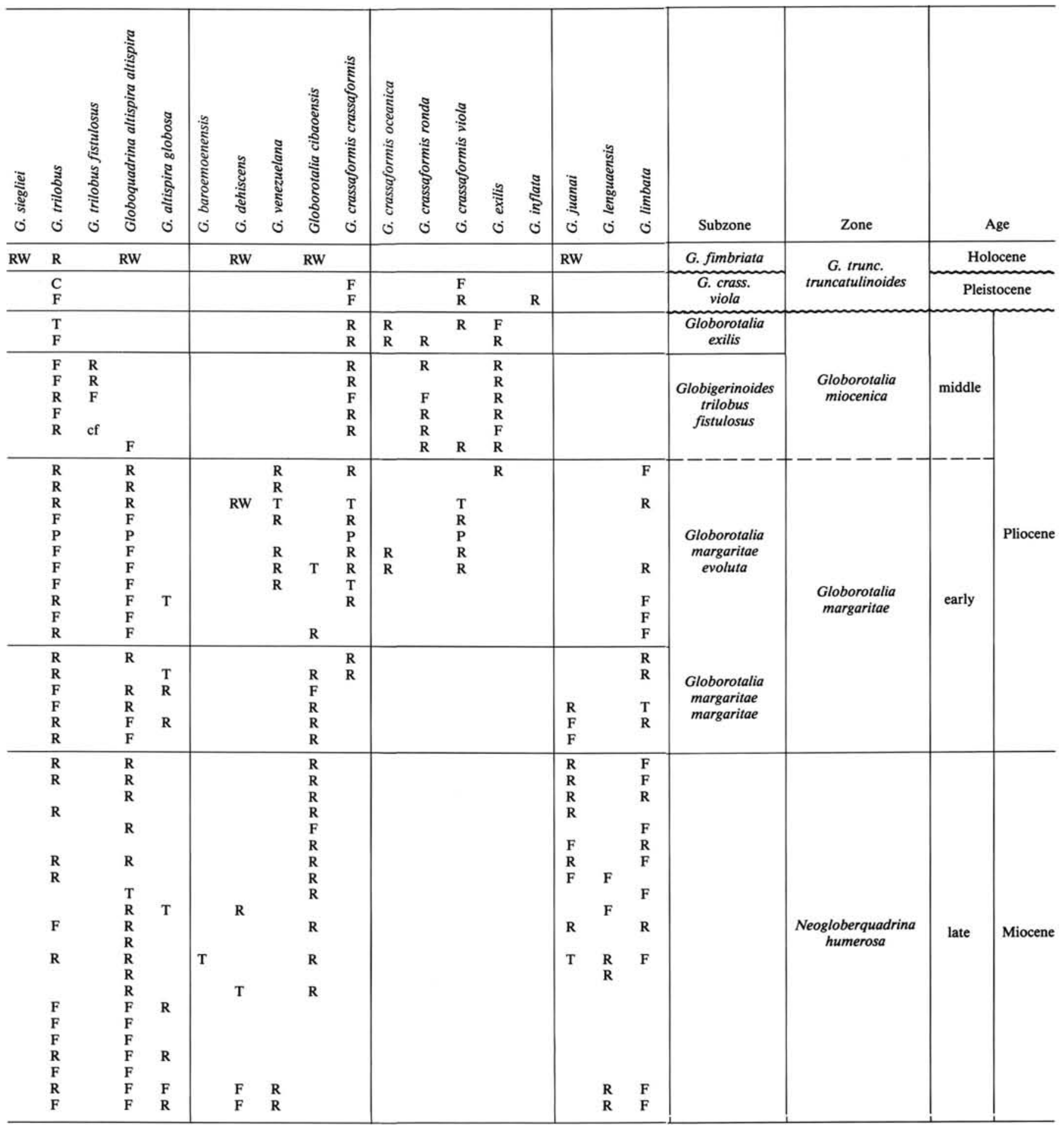

Figure 7 (continued).

zone is also missing (Figs. 8, 9, and 10). This suggests that an erosional event near the Pliocene/Pleistocene boundary produced hiatuses at Sites 632 and 633 (and possibly 631). A later event occurred in the early Pleistocene that resulted in an unconformity at Site 631 , with minor hiatuses at Sites 632 and 633 or none at all.

Sample 101-631A-3H-3, 25-27 cm, correlates with the middle Pliocene Globorotalia miocenica Zone, based on the occurrence of the nominate taxon and $G$. exilis (Fig. 10). The pres- ence of Globigerinoides extremus suggests that the upper part of the $G$. exilis Subzone is missing (Fig. 10). This zone extends at least as far down as Section 101-631A-5H, CC, where $G$. miocenica first occurs (Fig. 10). The uppermost occurrence of Globoquadrina altispira altispira also occurs in this sample and was taken to indicate the top of the Globigerinoides trilobus fistulosus Subzone (Fig. 10; Bolli and Saunders, 1985). Thus, the middle Pliocene sediments above this level (i.e., Samples 101$631 \mathrm{~A}-5 \mathrm{H}-3,25-27 \mathrm{~cm}$, through 101-631A-3H-3, 25-27 cm) cor- 


\begin{tabular}{|c|c|c|c|c|c|c|c|c|c|c|c|c|c|c|c|c|c|c|c|c|c|c|c|c|}
\hline $\begin{array}{c}\text { Hole } 630 \mathrm{~A} \\
\text { Core-section } \\
\text { (interval in cm) }\end{array}$ & 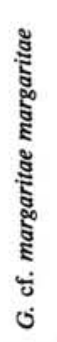 & 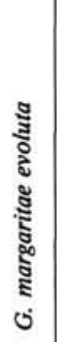 & 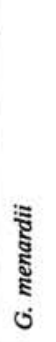 & 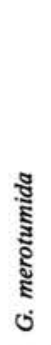 & 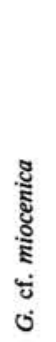 & 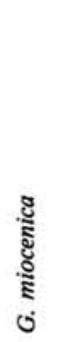 & 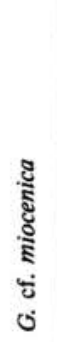 & 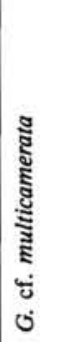 & 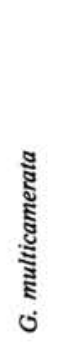 & 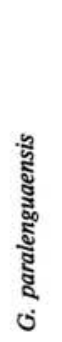 & 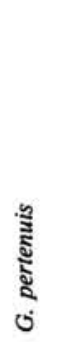 & 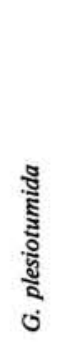 & 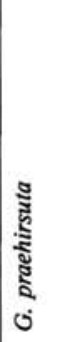 & 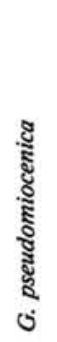 & 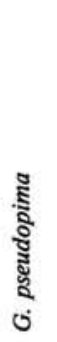 & 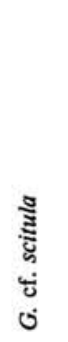 & 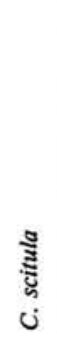 & 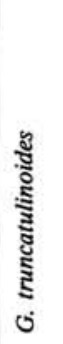 & 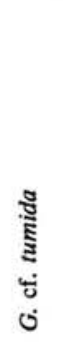 & 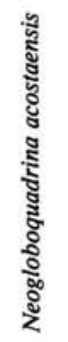 & 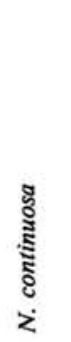 & 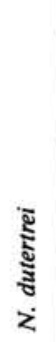 & 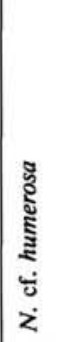 & 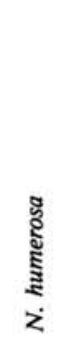 \\
\hline $1 \mathrm{H}-3,25-27$ & & & & $\mathbf{R}$ & & & & & & & & & & & & & & $\mathbf{R}$ & & & & & & \\
\hline $\begin{array}{l}1 \mathrm{H}-4,25-27 \\
1 \mathrm{H}, \mathrm{CC}\end{array}$ & & & & & & & & & & & & & & & & & & $\begin{array}{l}\mathrm{R} \\
\mathrm{F} \\
\end{array}$ & $T$ & & - & $\begin{array}{l}F \\
F\end{array}$ & & \\
\hline $\begin{array}{l}2 \mathrm{H}-4,25-27 \\
2 \mathrm{H}, \mathrm{CC}\end{array}$ & & & & & & & $\begin{array}{l}\mathrm{F} \\
\mathrm{R}\end{array}$ & & & & & & & & & $T$ & & & & $\mathbf{R}$ & & $\begin{array}{l}\mathbf{R} \\
\mathbf{R}\end{array}$ & & $\mathbf{R}$ \\
\hline $\begin{array}{l}3 \mathrm{H}-4,25-27 \\
3 \mathrm{H}, \mathrm{CC} \\
4 \mathrm{H}-3,25-27 \\
4 \mathrm{H}, \mathrm{CC} \\
5 \mathrm{H}-3,25-27 \\
5 \mathrm{H}, \mathrm{CC} \\
\end{array}$ & & & & $\mathbf{R}$ & & $\mathrm{F}$ & $\begin{array}{l}\mathrm{F} \\
\mathrm{F} \\
\mathrm{F} \\
\mathrm{F} \\
\mathrm{R}\end{array}$ & & $\begin{array}{l}\mathbf{R} \\
\mathbf{R}\end{array}$ & & $\begin{array}{l}\mathbf{R} \\
\mathrm{R} \\
\mathrm{R}\end{array}$ & $\mathrm{RW}$ & & & $\mathbf{R}$ & $\mathbf{R}$ & $T$ & & & $\mathbf{R}$ & & $\mathbf{R}$ & $\begin{array}{l}\mathbf{R} \\
\mathbf{R} \\
\end{array}$ & $\begin{array}{l}\mathrm{R} \\
\mathbf{R} \\
\mathrm{F} \\
\mathbf{R}\end{array}$ \\
\hline $\begin{array}{l}6 \mathrm{H}-4,25-27 \\
6 \mathrm{H}, \mathrm{CC} \\
7 \mathrm{H}-4,25-27 \\
7 \mathrm{H}, \mathrm{CC} \\
8 \mathrm{H}-3,25-27 \\
8 \mathrm{H}, \mathrm{CC} \\
9 \mathrm{H}-3,25-27 \\
9 \mathrm{H}, \mathrm{CC} \\
10 \mathrm{H}-3,25-27 \\
10 \mathrm{H}, \mathrm{CC} \\
11 \mathrm{H}-3,25-27 \\
\end{array}$ & $\begin{array}{l}\mathrm{T} \\
\mathrm{R}\end{array}$ & $\begin{array}{l}\mathbf{F} \\
\mathbf{R} \\
\mathbf{F} \\
\mathbf{R}\end{array}$ & $\begin{array}{l}\text { R } \\
\text { cf } \\
\text { R } \\
\text { F }\end{array}$ & $\mathrm{R}$ & & $\begin{array}{l}\mathrm{T} \\
\mathrm{R} \\
\mathrm{R} \\
\mathrm{T} \\
\\
\\
\mathrm{T}\end{array}$ & $\mathrm{T}$ & C & $\begin{array}{l}\mathbf{R} \\
\mathrm{R}\end{array}$ & & & $\begin{array}{l}\text { cf } \\
\\
\\
\text { R } \\
T \\
T\end{array}$ & R & $\begin{array}{l}\mathrm{R} \\
\mathrm{R}\end{array}$ & $\begin{array}{l}\mathbf{R} \\
\mathbf{R}\end{array}$ & & & & & $\begin{array}{l}\mathrm{R} \\
\\
\mathrm{R} \\
\mathrm{P} \\
\mathrm{R} \\
\mathrm{R} \\
\mathrm{R} \\
\mathrm{R} \\
\mathrm{T}\end{array}$ & & & $\mathbf{R}$ & $\begin{array}{l}\mathrm{R} \\
\mathrm{R} \\
\mathrm{R} \\
\mathrm{F} \\
\mathrm{R} \\
\mathrm{R} \\
\mathrm{R}\end{array}$ \\
\hline $\begin{array}{l}11 \mathrm{H}, \mathrm{CC} \\
12 \mathrm{H}-3,25-27 \\
12 \mathrm{H}, \mathrm{CC} \\
13 \mathrm{H}-3,25-27 \\
13 \mathrm{H}, \mathrm{CC} \\
14 \mathrm{H}-3,25-27 \\
\end{array}$ & $\begin{array}{l}\mathbf{R} \\
\mathbf{R} \\
\mathbf{R} \\
\end{array}$ & $\begin{array}{l}\mathrm{R} \\
\mathrm{R} \\
\mathrm{F} \\
\\
\mathrm{R} \\
\mathrm{R}\end{array}$ & & & & & & $\begin{array}{l}\mathbf{R} \\
\mathbf{F} \\
\mathbf{R} \\
\mathbf{R} \\
\mathbf{R} \\
\mathrm{F} \\
\end{array}$ & & & & $\mathbf{R}$ & & & & & & & & $\begin{array}{l}\mathrm{R} \\
\mathrm{R} \\
\mathrm{R} \\
\mathrm{R} \\
\mathrm{R} \\
\mathrm{R} \\
\end{array}$ & $\mathbf{R}$ & & & $R$ \\
\hline $\begin{array}{l}14 \mathrm{H}, \mathrm{CC} \\
15 \mathrm{H}, \mathrm{CC} \\
16 \mathrm{H}-3,25-27 \\
16 \mathrm{H}, \mathrm{CC} \\
17 \mathrm{H}-3,25-27 \\
17 \mathrm{H}, \mathrm{CC} \\
18 \mathrm{H}-3,25-27 \\
18 \mathrm{H}, \mathrm{CC} \\
19 \mathrm{X}-3,25-27 \\
19 \mathrm{X}, \mathrm{CC} \\
20 \mathrm{X}-3,25-27 \\
20 \mathrm{X}, \mathrm{CC} \\
21 \mathrm{X}-3,25-27 \\
21 \mathrm{X}, \mathrm{CC} \\
22 \mathrm{X}, \mathrm{CC} \\
23 \mathrm{X}-3,25-27 \\
23 \mathrm{X}, \mathrm{CC} \\
24 \mathrm{X}-3,25-27 \\
24 \mathrm{X}, \mathrm{CC} \\
25 \mathrm{X}, \mathrm{CC} \\
26 \mathrm{X}-3,25-27 \\
26 \mathrm{X}, \mathrm{CC}\end{array}$ & $\mathrm{T}$ & & & $\begin{array}{l}\text { R } \\
\mathrm{T} \\
\mathrm{F} \\
\mathrm{F} \\
\mathrm{R}\end{array}$ & $\begin{array}{l}\mathrm{F} \\
\mathrm{F} \\
\mathrm{R} \\
\mathrm{F} \\
\mathrm{R}\end{array}$ & & & $\begin{array}{l}R \\
F \\
F\end{array}$ & $\begin{array}{l}\mathbf{R} \\
\mathbf{R}\end{array}$ & $\mathrm{R}$ & & $\begin{array}{l}\mathrm{R} \\
\mathrm{R} \\
\mathrm{T} \\
\mathrm{R} \\
\mathrm{R} \\
\mathrm{F} \\
\\
\\
\mathrm{R} \\
\mathrm{R} \\
\mathrm{R} \\
\mathrm{R} \\
\mathrm{R} \\
\\
\\
\\
\mathrm{F} \\
\mathrm{R} \\
\mathrm{R}\end{array}$ & & & & $\mathrm{T}$ & $\begin{array}{l}\mathrm{R} \\
\\
\mathrm{R} \\
\mathrm{R} \\
\mathrm{R} \\
\mathrm{R} \\
\mathrm{R} \\
\mathrm{R} \\
\mathrm{R} \\
\mathrm{R} \\
\mathrm{R} \\
\mathrm{R}\end{array}$ & & & $\begin{array}{l}\mathrm{R} \\
\mathrm{R} \\
\mathrm{R} \\
\mathrm{R} \\
\mathrm{R} \\
\mathrm{R} \\
\mathrm{R} \\
\mathrm{R} \\
\mathrm{F} \\
\mathrm{R} \\
\mathrm{R} \\
\mathrm{R} \\
\mathrm{R} \\
\mathrm{R} \\
\mathrm{R} \\
\mathrm{F} \\
\mathrm{R} \\
\mathrm{F} \\
\mathrm{F} \\
\mathrm{R} \\
\mathrm{F}\end{array}$ & $\begin{array}{l}\mathrm{T} \\
\mathrm{R} \\
\mathrm{T} \\
\mathrm{T}\end{array}$ & & $\begin{array}{l}\mathrm{R} \\
\mathrm{T} \\
\mathrm{T}\end{array}$ & $\mathrm{T}$ \\
\hline
\end{tabular}

Figure 7 (continued).

relate with the late middle Pliocene Globorotalia exilis Subzone on the basis of superposition (Fig. 10).

Bolli and Saunders (1985) showed the stratigraphic range of $G$. margaritae margaritae overlapping with that of $G$. miocenica in the uppermost part of the early Pliocene Globorotalia margaritae Zone. In Hole 631A a gap of $73.75 \mathrm{~m}$ (Samples 101-631A$5 \mathrm{H}, \mathrm{CC}$ to $101-631 \mathrm{~A}-13 \mathrm{X}-3,25-27 \mathrm{~cm}$ ) occurs between the LO of G. margaritae margaritae and the FO of G. miocenica (Fig. 10). This is almost certainly due to the poor preservation char- acterizing this interval (Fig. 10). According to Bolli and Saunders (1985), the LO of Globoquadrina venezuelana (Hedberg) is at the top of the Globorotalia margaritae Zone. Thus, the top of the Globorotalia margaritae Zone was considered to extend to the level of Sample 101-631A-10H-3, 25-27 cm, where $G$. venezuelana last occurs (Fig. 10). The sporadic occurrence of this species below Sample 101-631A-10H-3, 25-27 cm, may suggest reworking and, hence, placement of the boundary was only tentative. 


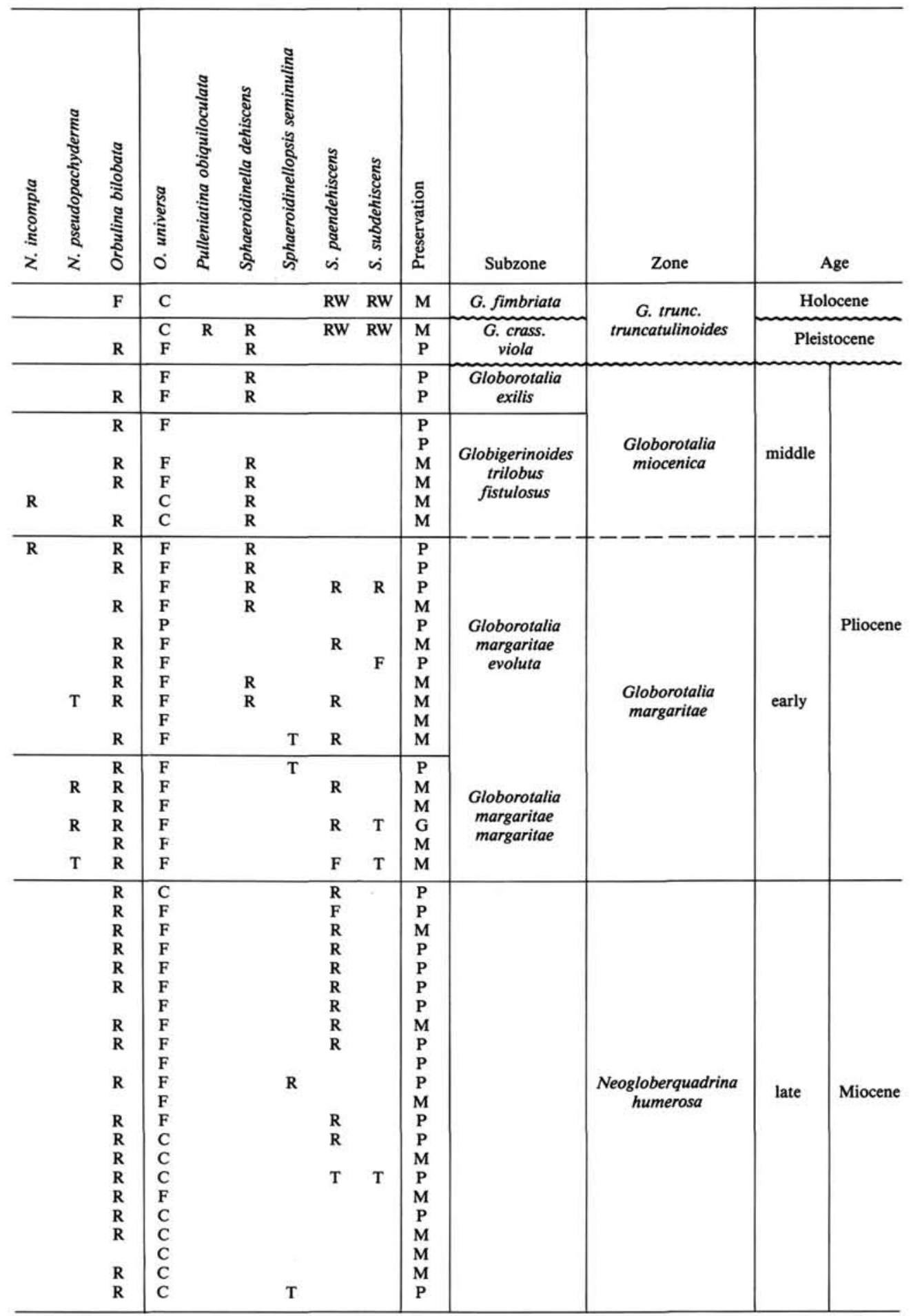

Figure 7 (continued).

The presence of Globorotalia margaritae margaritae and $G$. margaritae evoluta in Section 101-631A-25X, CC at the bottom of the hole suggests an age of late early Pliocene (Globorotalia margaritae evoluta Subzone) (Fig. 10). Based on the paucity of the former species above this level and the absence of the latter, these specimens were not considered downhole contaminants (Fig. 10). Based on superposition, the sediments in the interval above Section 101-631A-25X, CC (i.e., Section 101-631A-24X, $\mathrm{CC}$ through Sample 101-631A-10H-3, 25-27 cm) thus are in the late early Pliocene Globorotalia margaritae evoluta Subzone (Fig. 10).

\section{UNCONFORMITIES AND SEA-LEVEL CHANGES}

One of the main objectives of Leg 101 was to document the relationship between sea-level changes and carbonate platform shedding (Austin, Schlager, et al., 1986). Vail et al. (1977) tacitly suggested that carbonate systems respond to lowered sea levels in the same manner that siliciclastic margins do; that is, by in- 


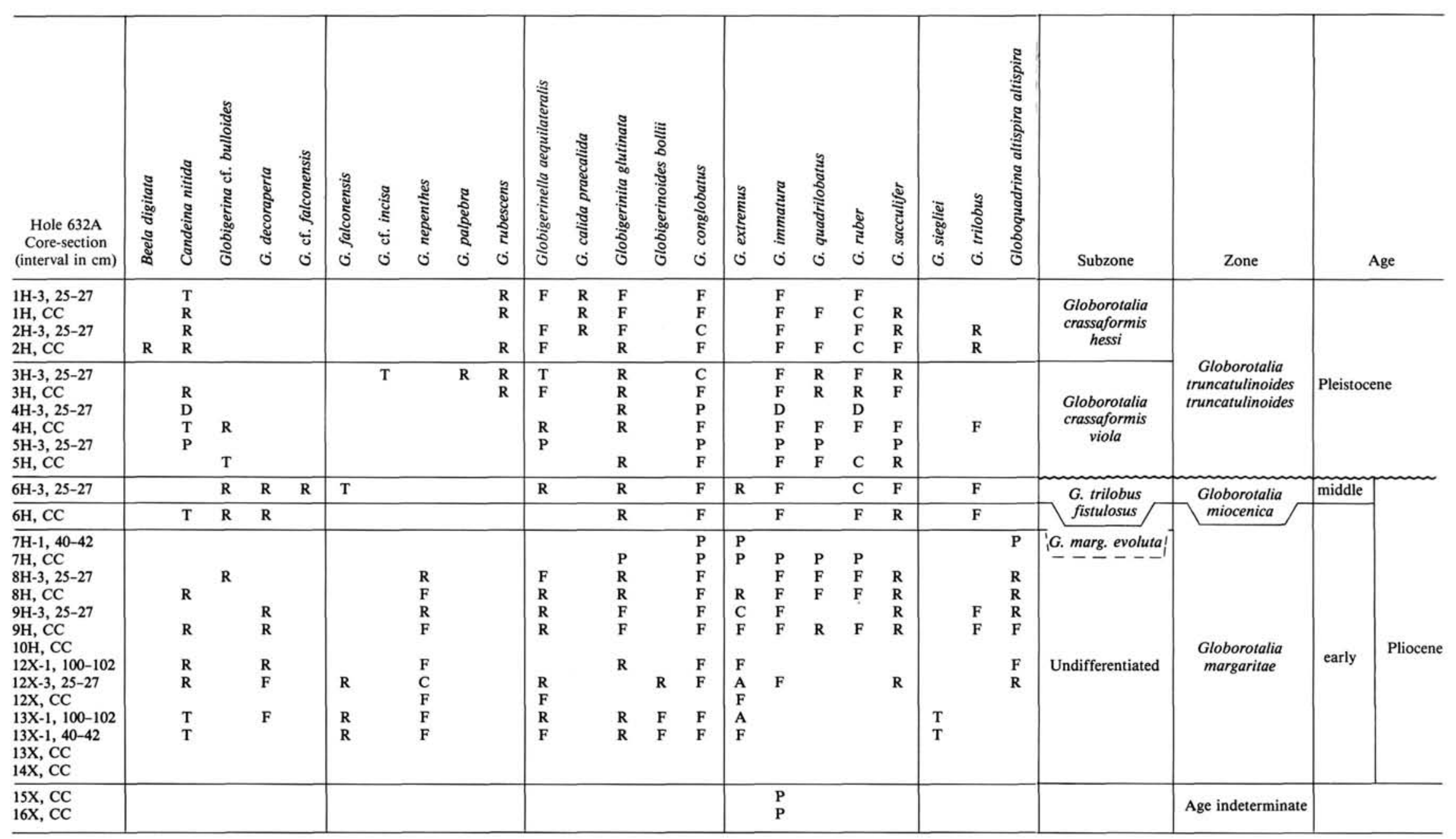

Figure 8. Stratigraphic ranges of selected planktonic foraminifers at Hole 632A. 


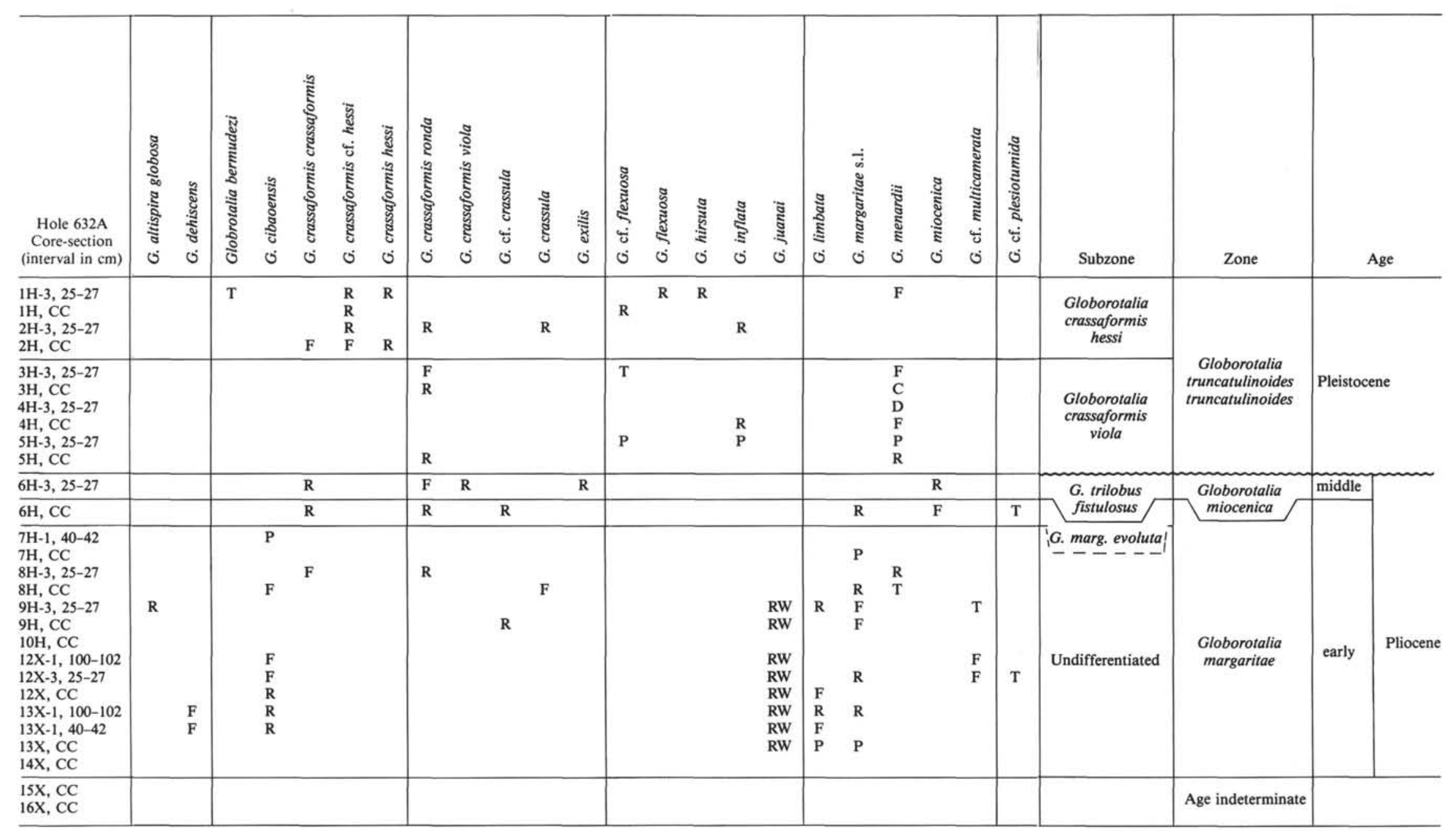

Figure 8 (continued). 


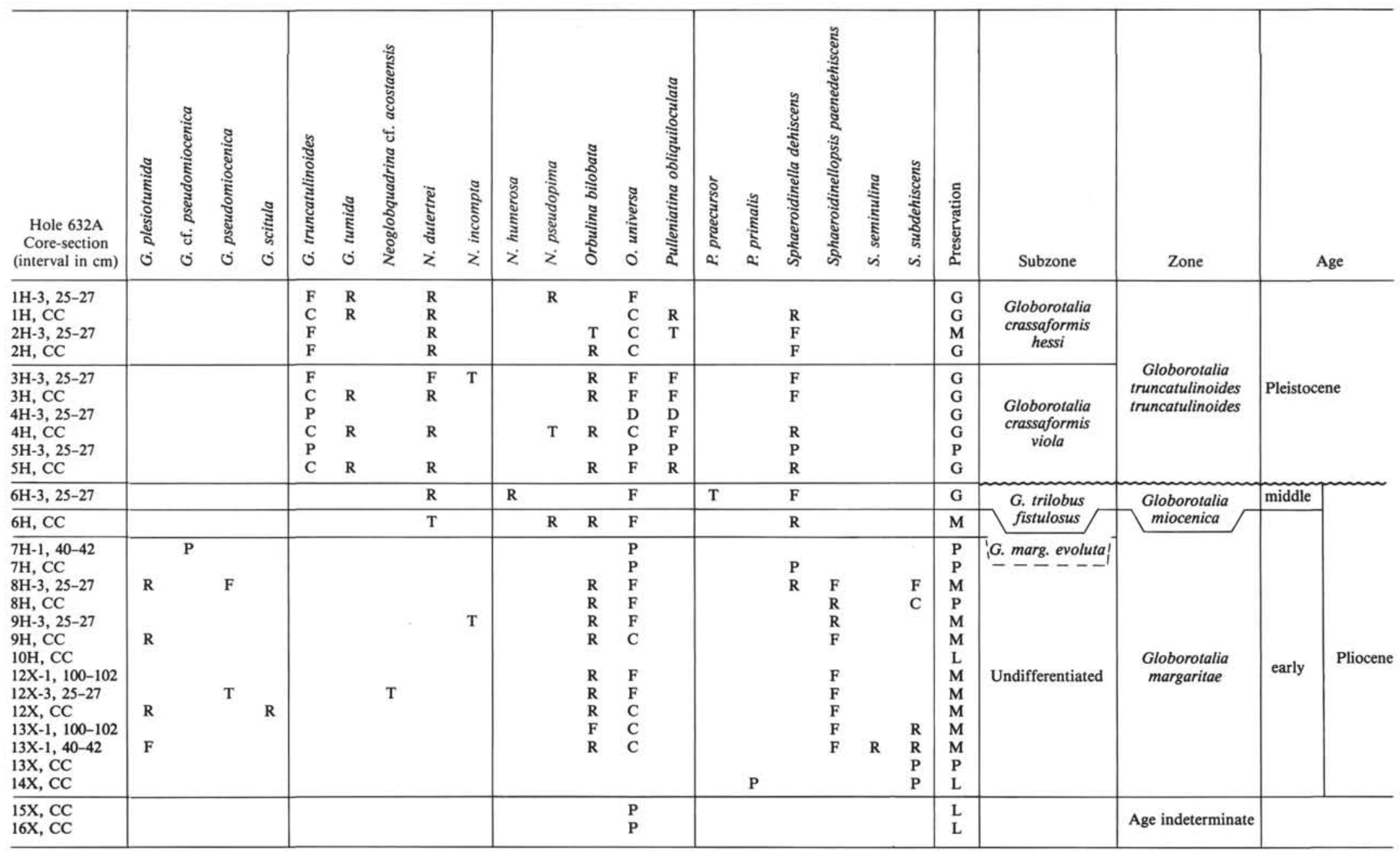

Figure 8 (continued). 
creasing the amount of sediment transported from the carbonate bank to the adjacent basin. Other scientists, including Vail and Hardenbol (1979), Vail et al. (1980), and Haq et al. (1987), also implied this. Studies by Thiede (1981) and Shanmugam and Moiola $(1982,1983,1984)$ generally support this view. However, other studies (Kier and Pilkey, 1971; Lynts et al., 1973; Schlager and Chermak, 1979; Mullins et al., 1980; Schlager and Ginsburg, 1981; Droxler et al., 1983; Boardman and Neumann, 1984; Droxler and Schlager, 1985; Boardman et al., 1986; and others) suggested that carbonate platforms shed the greatest amount of material during periods of elevated sea level.

An increase in the frequency of sediment gravity flows on siliciclastic margins during periods of lowered sea level is well documented (Bé et al., 1976; Damuth, 1977; Vail et al., 1977; Shanmugam and Moiola, 1982). Other studies have identified unconformities in the North Atlantic continental margin that correspond to many of the sea-level decreases proposed by Haq et al. (1987) in the most recent published version of the coastal onlap chart of Vail et al. (Melillo, 1985; Miller et al., 1985a, 1987; Miller and Hart, 1987; Olsson et al., 1987; Olsson and Wise, 1987; Poag and Low, 1987). A similar correlation between the timing of postulated lowered sea levels and erosional unconformities should occur in the Bahamas if carbonate banks respond to sea-level changes in the same ways as siliciclastic margins. However, localized mechanisms, such as scouring caused by fluctuations in the velocity of the Gulf Stream or by eddies that periodically spin off the main current, also may be important in producing unconformities (Austin, Schlager, et al., 1986). In addition, variations in bank topography can also affect the timing of platform shedding events (Moore et al., 1976). Thus, a number of factors can obscure any eustatic signal.

In addition to revising and updating the onlap-offlap curve of Vail et al. (1977, 1980), Haq et al. (1987) also presented a eustatic curve that they correlated with the planktonic foraminifer zonation of Stainforth et al. (1975). Figure 11 displays these correlations as well as the slightly modified planktonic foraminifer zonation of Bolli and Saunders (1985) used here. Both planktonic zonations are based largely on the works of Bolli (1957, 1966), Bolli and Bermudez (1965), and Bolli and Premoli-Silva (1973) and share many zonal criteria and taxonomic concepts and thus can be correlated easily (Fig. 11). This is especially true for the late Oligocene and Miocene, where only several minor differences exist (Fig. 11). The first difference is the aforementioned use of an undifferentiated Catapsydrax dissimilis/C. stainforthi Zone in this study. A second difference is a slightly lower position for the base of the Globorotalia fohsi fohsi Zone of Stainforth et al. as a result of the inclusion of $G$. fohsi peripheroacuta in their concept of $G$. fohsi fohsi (Fig. 11). Other differences are caused by the use of additional zones and subzones identified by Bolli and Saunders (Fig. 11). The late middle Miocene Globigerinoides ruber Zone used here is correlated with the lower Globorotalia siakensis Zone of Stainforth et al. on the basis of range of Globigerina nepenthes given by Stainforth et al. and Bolli and Saunders (Fig. 11). The late Miocene Neogloboquadrina humerosa Zone is correlated with the upper Globorotalia acostaensis Zone of Stainforth et al., based on the ranges of $N$. humerosa and Candeina nitida (Fig. 11).

The early Pliocene Globorotalia margaritae Zone used here correlates with the G. margaritae Zone of Stainforth et al. (1975) (Fig. 11). However, my correlations of these subzones are tentative. The zonal criteria used by Stainforth et al. and Bolli and Saunders (1985) also differ markedly in the remainder of the Pliocene and Pleistocene. In this interval, correlations with the Stainforth et al. zonation and, hence, with the eustatic curve of Haq et al. (1987), are tenuous (Fig. 11).

\section{Major Unconformities}

In Hole 628A, a major unconformity is present in the upper part of Core 101-628A-16H. A thin limestone (Sample 101-628A$16 \mathrm{H}-1,9-30 \mathrm{~cm}$ ) separates the periplatform oozes and slump deposits of lithologic Subunit ID from the underlying, oceanicplateau, nannofossil-foraminifer oozes of lithologic Unit II (Austin, Schlager, et al., 1986). This lithologic break corresponds to a major hiatus and to the boundary between seismic sequences C and D (Austin, Schlager, et al., 1986).

Sediments above the unconformity are of middle Miocene age, correlative with the middle to upper Globorotalia fohsi lobata Zone, while those below correlate with the late Oligocene Globigerina ciperoensis Zone (Fig. 11). This indicates a hiatus of approximately $11.4 \mathrm{~m} . y$., using the time scale of Berggren et al. (1985) with modifications of Miller et al. (1985b).

The eustatic curve of Haq et al. (1987) indicates a high sealevel stand (cycle TB2.5) that corresponds to the middle to upper Globorotalia fohsi lobata Zone (Fig. 11). Thus, the erosional event at Hole 628A apparently occurred during an interval of elevated sea level (Fig. 11). Austin, Schlager, et al. (1986) related the slump to a regional, probably tectonic, event.

At Hole 627B, a major unconformity separates lowermost Neogene sediments from Paleogene sediments and probably correlates with the seismic sequence C/D boundary (Fig. 11; Austin, Schlager, et al., 1986). The basal part of lithologic Subunit IC in Core 101-627B-19X is a lowermost Miocene (Globorotalia kugleri Zone) packstone of debris-flow origin that was deposited at the toe-of-slope of Little Bahamas Bank (Fig. 11; Austin, Schlager, et al., 1986). The upper part of Core 101-627B-20X contains the same age sediments, which probably are caved (Austin, Schlager, et al., 1986). The core-catcher sample contains a chert nodule that represents part of a middle to upper Eocene (Morozovella lehneri Zone to Turborotalia cerroazulensis s.l. Zone) siliceous limestone condensed section, indicating a hiatus of approximately 12.1-18.5 m.y. (Figs. 5 and 11).

Recovery across this unconformity probably was incomplete (Austin, Schlager, et al., 1986). As a result, it is difficult to determine the exact age of the event that produced the unconformity. However, the lack of diagnostic Oligocene planktonic foraminifers suggests that this event was in the earliest Miocene (Globorotalia kugleri Zone). Austin, Schlager, et al. (1986) correlated the compound gravity-flow facies of Subunit IC with part of the Great Abaco Member of the Blake Ridge Formation and with sediment gravity flows at Site 626 in the Straits of Florida. Based on the large areal distribution of these deposits, they proposed a tectonic and/or sea-level event of at least regional extent. The eustatic curve of Haq et al. (1987) showed an elevated sea level (cycle TB1.4) that corresponds to the earliest Miocene Globorotalia kugleri Zone (Fig. 11). Thus, this unconformity may also have been formed during a period of high sea level (Fig. 11).

Upper Pliocene (Globorotalia tosaensis tosaensis Zone) sediments are absent at all Leg 101 sites. This is indicated by the lack of any stratigraphic gap between the LO of G. miocenica and the FO of $G$. truncatulinoides truncatulinoides (Figs. 3-10). The unconformity does not appear to be an artifact of reworking of $G$. miocenica or of sample spacing. Thus, it may be a regional feature. Correlating this event with the eustatic curve of Haq et al. (1987) was difficult because they do not recognize the Globorotalia tosaensis tosaensis Zone. However, I believe that the hiatus correlates with the decreased sea levels of the lower part of cycle TB3.7 (Fig. 11).

Figure 11 shows that, aside from the upper Pliocene hiatus, there is no consistent pattern in the temporal distribution of hia- 


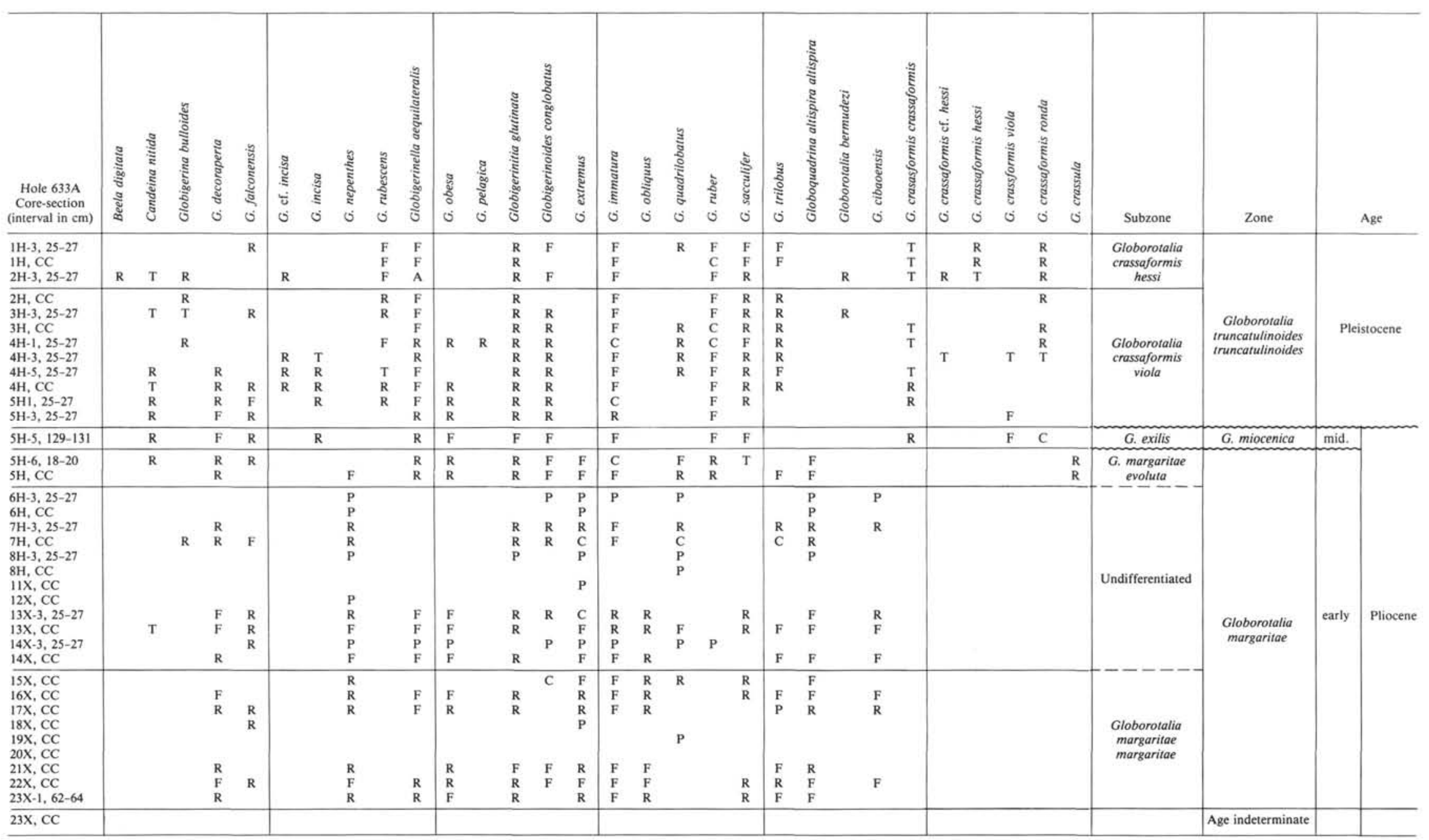

Figure 9. Stratigraphic ranges of selected planktonic foraminifers at Hole 633A. 


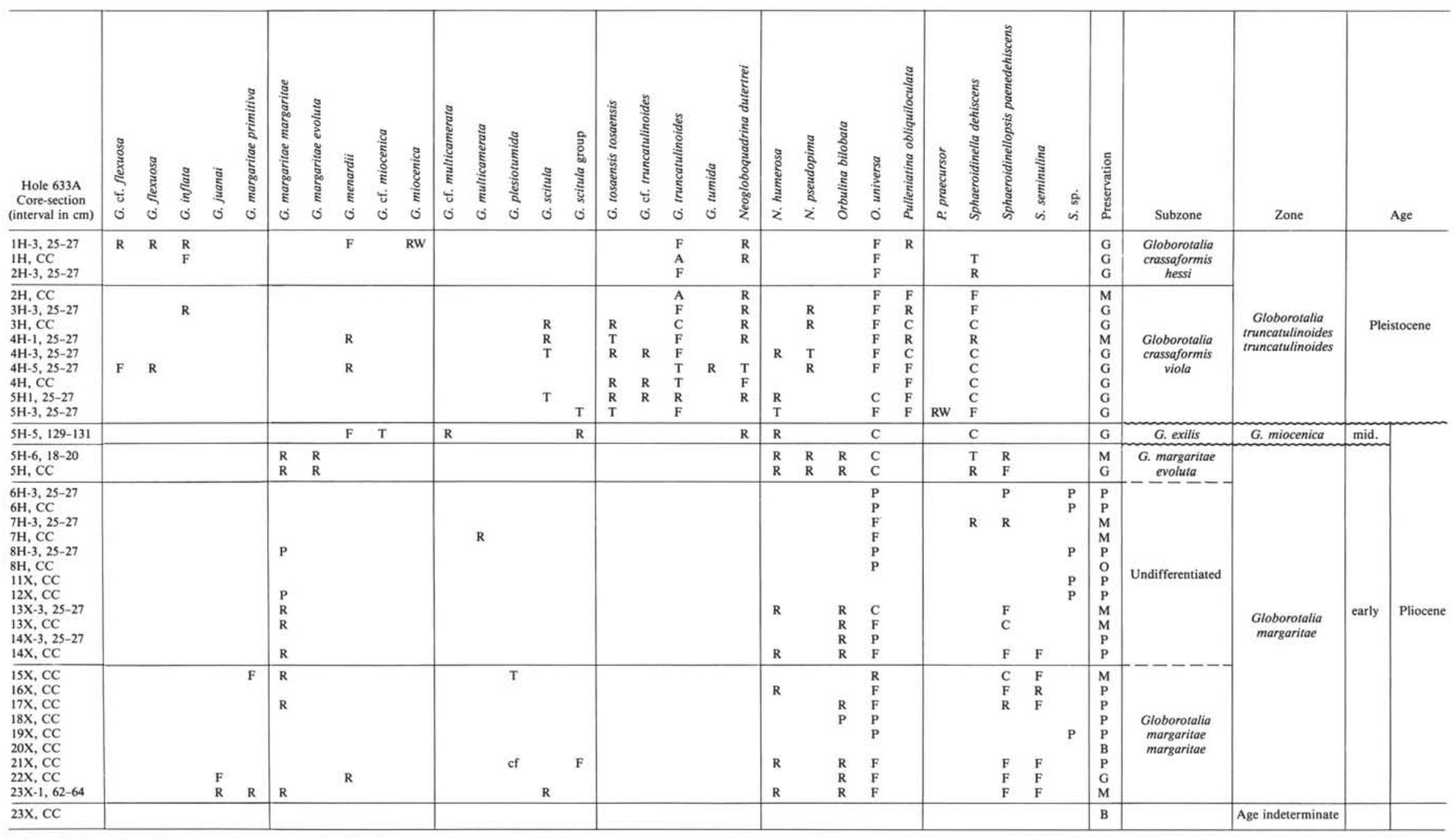

Figure 9 (continued). 


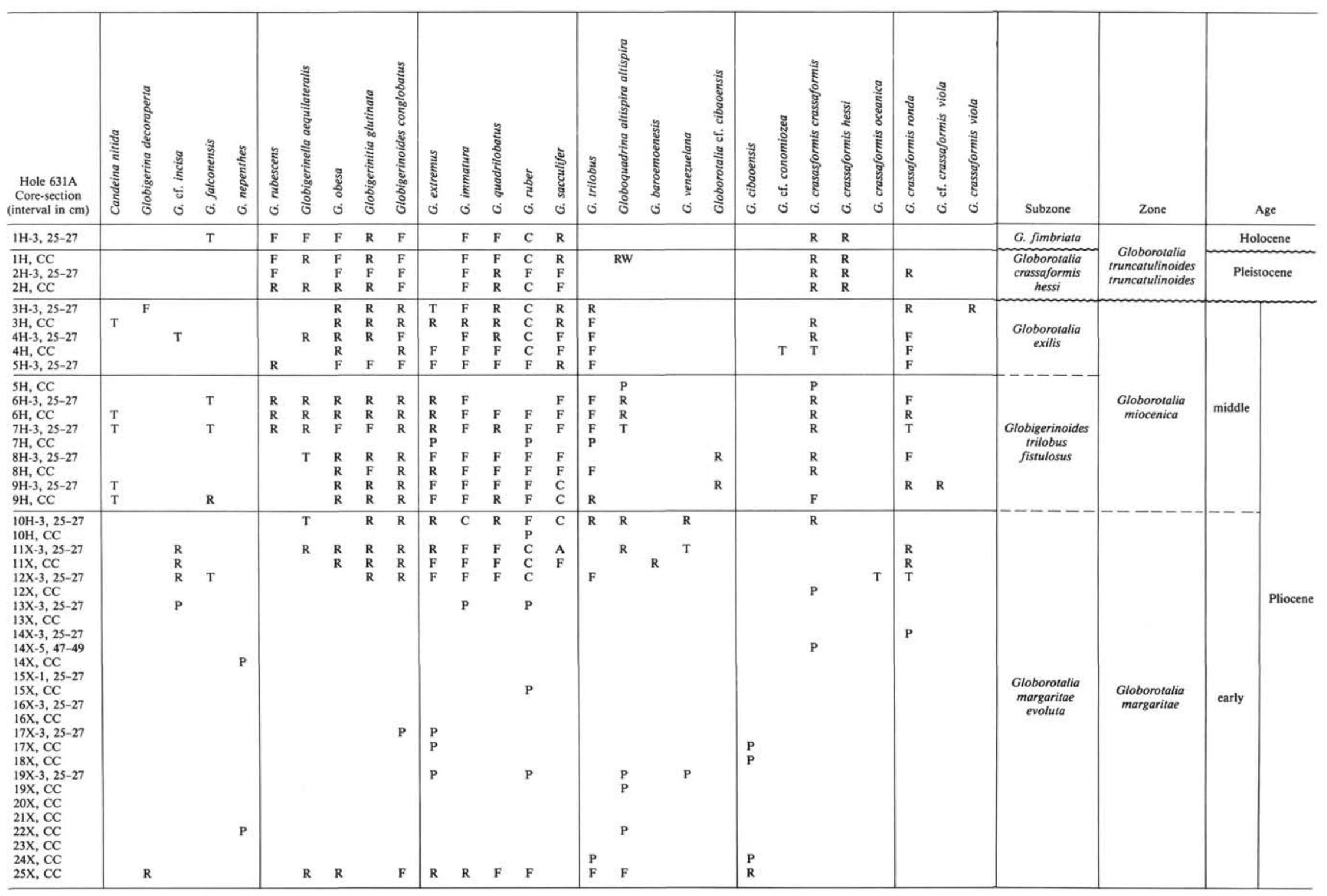

Figure 10. Stratigraphic ranges of selected planktonic foraminifers at Hole 631A. 


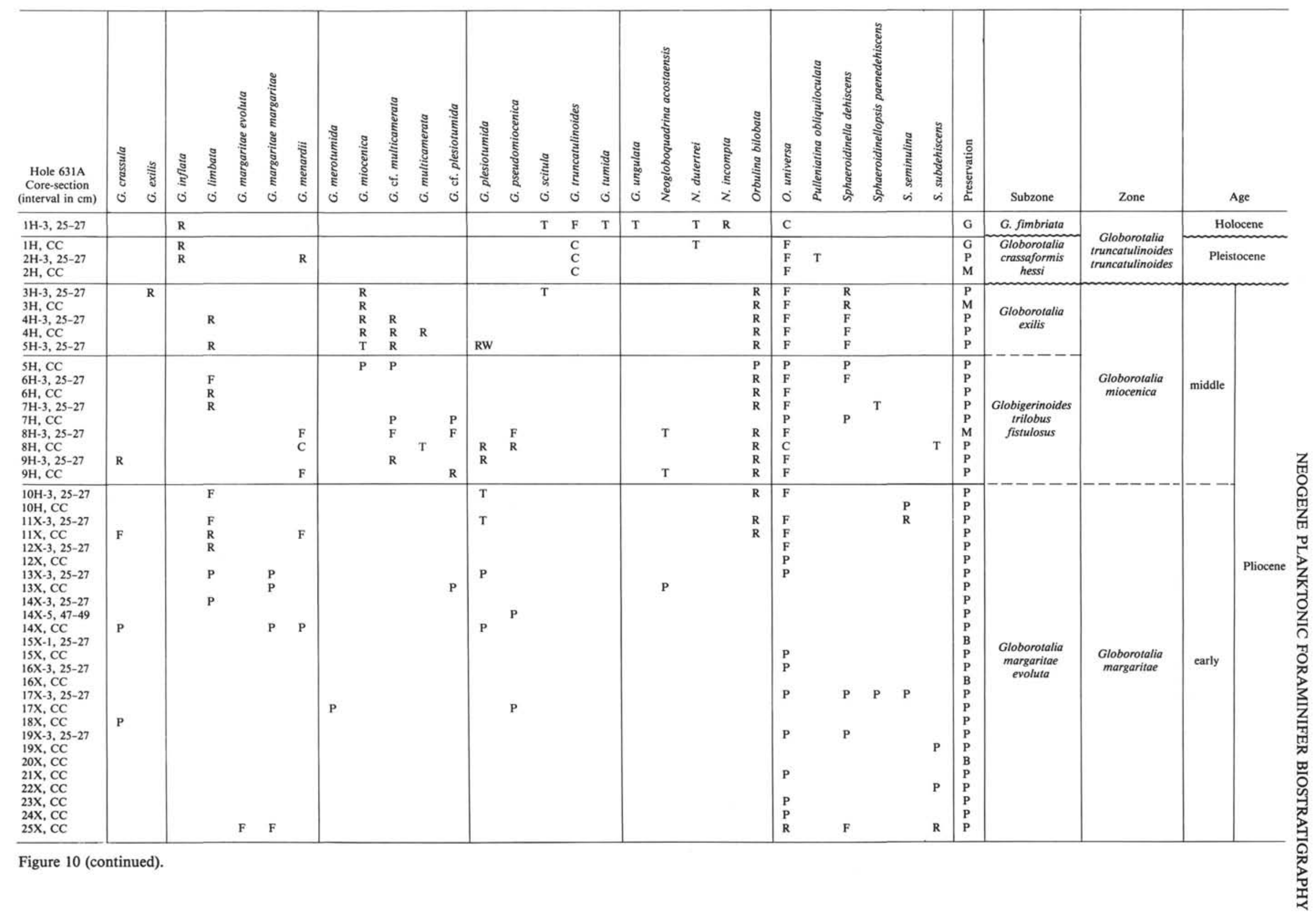




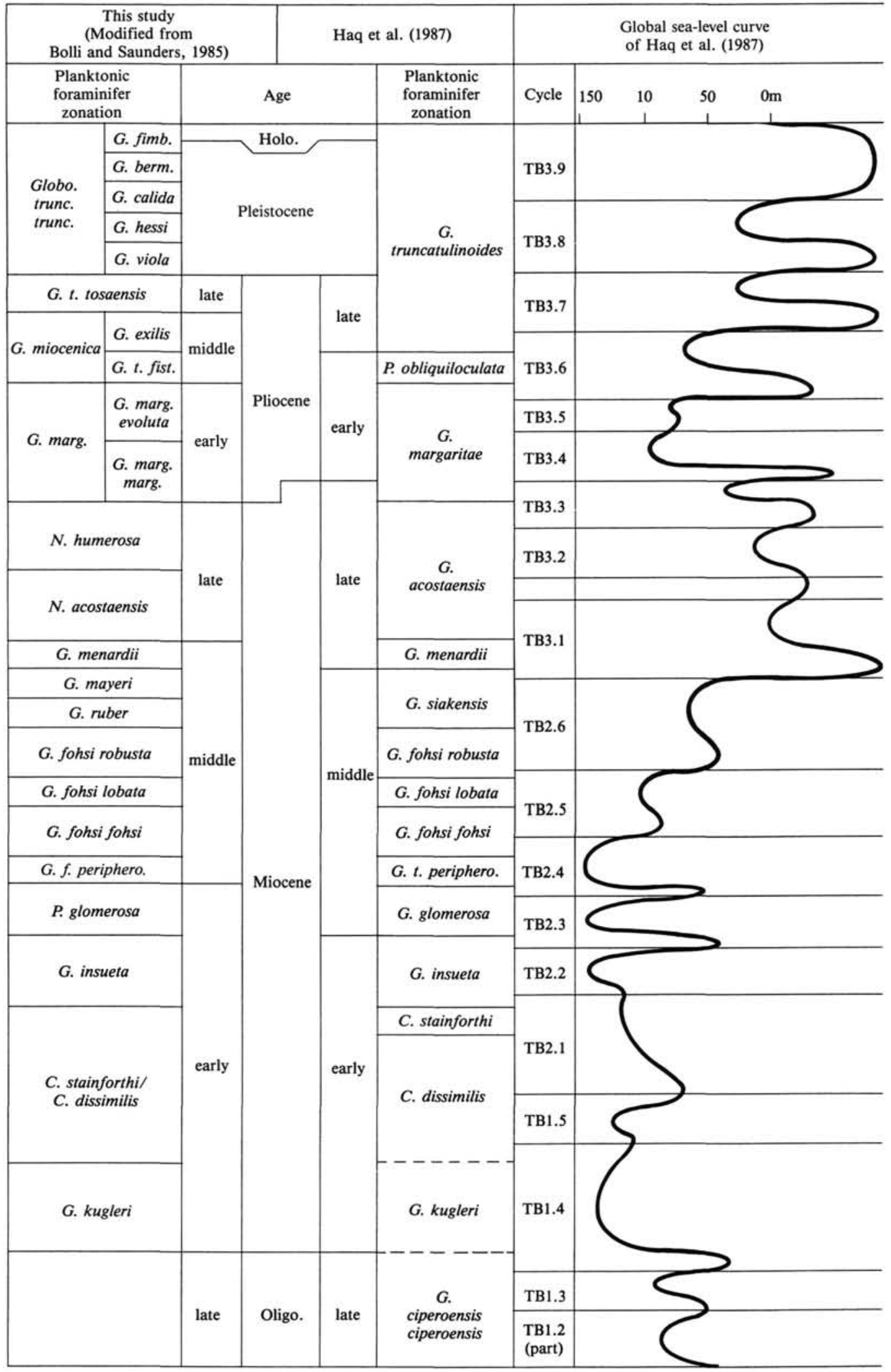

Figure 11. Comparison of Neogene hiatuses identified during Leg 101 with the eustatic curve of Haq et al. (1987). Shading indicates hiatuses. 
NEOGENE PLANKTONIC FORAMINIFER BIOSTRATIGRAPHY

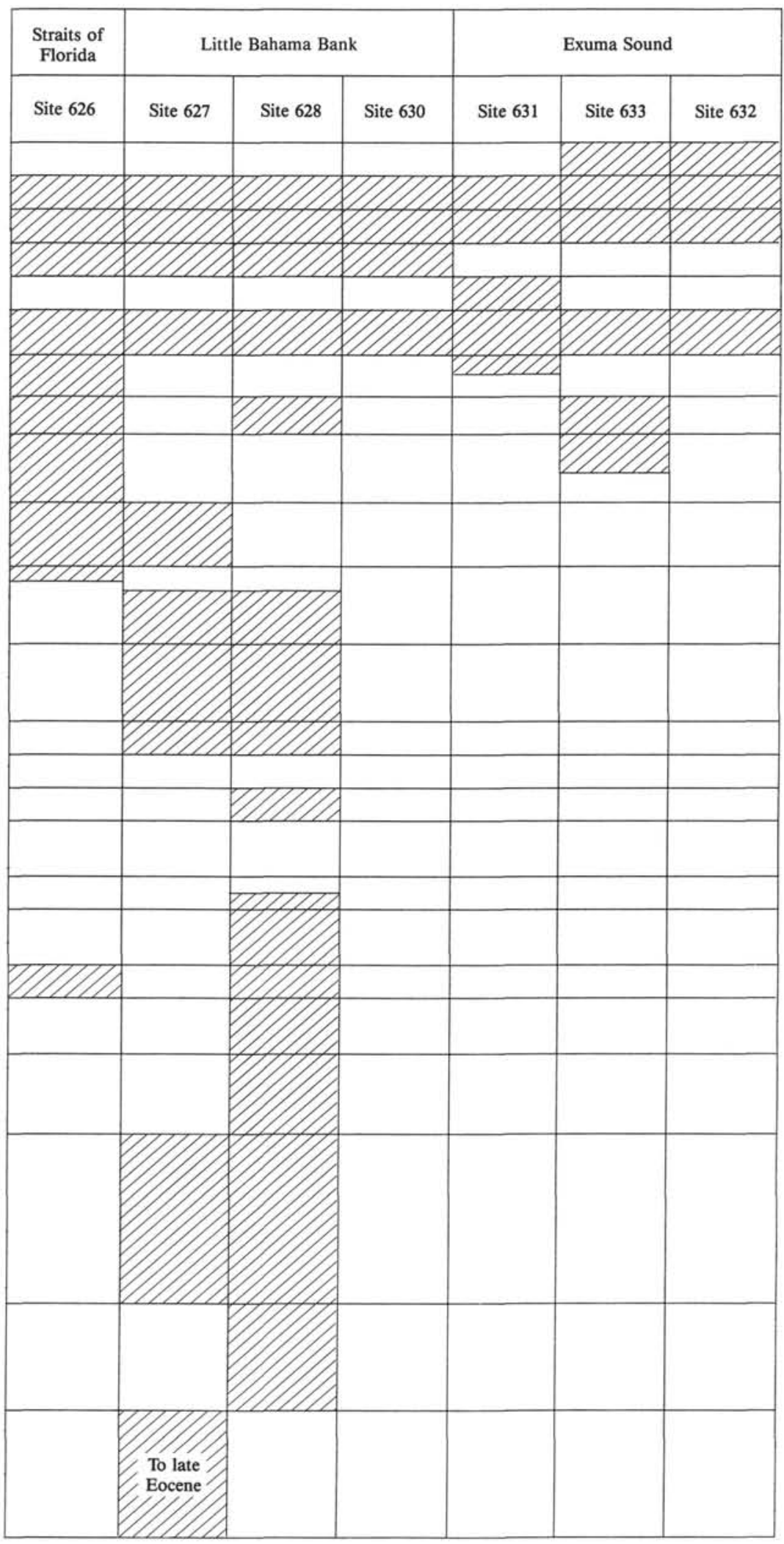

Figure 11 (continued). 
tuses. This suggests that more than one mechanism has produced hiatuses in this area. However, the largest hiatuses (Sites 627 and 628) may be associated with high sea levels (Fig. 11).

\section{CONCLUSIONS}

Site 626 in the Straits of Florida recovered a sequence of Holocene to upper Oligocene sediments (Fig. 3). Reworking was common in the Pleistocene-Holocene and hindered age determinations (Fig. 3). However, an unconformity is apparently present in which the uppermost Miocene and most of the Pliocene are missing (Fig. 3). Although sampling was sparse, a relatively conformable sequence of Miocene and uppermost Oligocene sediments was observed below this unconformity (Fig. 4).

Drilling in the Little Bahama Bank transect (Sites 627, 628, and 630) recovered sediments ranging in age from Holocene to middle to late Eocene (Figs. 5-7). Two major unconformities are present. One unconformity (at Hole 627B) may have resulted from an earliest Miocene (Globorotalia kugleri Zone) event that resulted in the removal of all, or almost all, of the Oligocene section (Fig. 5). The second unconformity (at Hole 628A) is associated with a middle Miocene (Globorotalia fohsi lobata Zone) event (Fig. 6). In both cases, the large hiatus may possibly be the result of coalesced unconformities, although this is not apparent in the available seismic data (Austin, Schlager et al., 1986). Correlation of the sediments immediately above these unconformities with the eustatic curve of Haq et al. (1987) suggests that erosion took place during periods of elevated sea levels. Thus, it may be that major carbonate platform-shedding events are associated with high sea levels, although tectonism or submarine erosion by currents cannot be excluded as causative or contributing factors.

Drilling at the Exuma Sound transect (Sites 631, 632, and 633) bottomed in the lower Pliocene (Figs. 8-10). Preservation at all three sites, especially Site 631 , was poor as a result of heavy carbonate overgrowth, dissolution, and lithification, probably caused by the influx of abundant platform-derived material (Figs. 8-10). At each site (except Hole 632A) a thick lower Pliocene section is present, along with a much reduced middle Pliocene section (Figs. 8-10).

The only unconformity that appears to be regional in extent is found in the upper Pliocene. Sediments of the Globorotalia tosaensis tosaensis Zone were not recovered at any Leg 101 site (Figs. 3-11). This minor hiatus may be associated with a period of lowered sea level. The overall lack of any systematic regional distribution of Neogene-Holocene hiatuses in the holes drilled during Leg 101 suggests that localized mechanisms are more important in producing unconformities than regional or largerscale mechanisms.

\section{ACKNOWLEDGMENTS}

I thank W. A. Berggren, J. Wright, J. A. Austin, Jr., A. A. Palmer, and W. Schlager for reviewing the final manuscript and offering helpful suggestions. Thanks also go to B. L. Hill for his review of an earlier version of the manuscript.

I thank W.P.S. Ventress for his support and Chevron U.S.A., Inc., for permission to publish this study and for the use of some of their personnel in preparing the manuscript. Special thanks go to E. Belsome and K. Savoy for typing the manuscript and to D. Greig for the scanning electron micrographs. D. Koffskey, S. Tran, and T. Cook assisted in preparing the plates. A. E. Young and J. R. De Ville did the original artwork.

I thank R. K. Olsson, C. S. Fulthorpe, and H. T. Mullins for helpful discussions of the biostratigraphy and sedimentology of the Bahamas.

Funding for most of this study was provided by USSAC, and their support is gratefully acknowledged. Much of the research was conducted under a post-doctoral fellowship in the Department of Geological Sciences, Rutgers University. This support is appreciated.

\section{REFERENCES}

Austin, J. A., Schlager, W., et al., 1986. Proc. ODP, Init. Repts., 101: College Station, TX (Ocean Drilling Program).

Bè, A.W.H., Damuth, J. E., Lott, L., and Free, R., 1976. Late Quaternary climatic record in western Equatorial Atlantic sediment. In Cline, R. M., and Hays, J. B. (Eds.), Investigation of Late Quaternary Paleoceanography and Paleoclimatology. Geol. Soc. Am. Mem., 145: 165-200.

Berggren, W. A., Kent, D. V., Flynn, J. J., and Van Couvering, J. A., 1985. Cenozoic geochronology. Geol. Soc. Am. Bull., 96: 1407-1418.

Boardman, M. R., and Neumann, A. C., 1984. Sources of periplatform carbonate: Northwest Providence Channel, Bahamas. J. Sediment. Petrol., 50:1121-1148.

Boardman, M. R., Neumann, A. C., Baker, P. A., Dulin, L. A., Kenter, R. J., Hunter, G. E., and Kiefer, K. B., 1986. Banktop responses to Quaternary fluctuations in sea-level recorded in periplatform sediments. Geology, 14:28-31.

Bolli, H. M., 1957. Planktonic foraminifera from the Oligocene-Miocene Cipero and Lengua formations of Trinidad, B.W.I. In Loeblich, A. R., Jr., Tappan, H., Beckmann, J. P., Bolli, H. M., Gallitelli, E. M., and Troelsen, J. C., Studies in Foraminifera. U.S. Nat. Mus. Bull., 215:97-124.

1966. Zonation of Cretaceous to Pliocene marine sediments based on planktonic foraminifera. Bol. Infor. Asoc. Venez. Geol., Min. Pet., 9:3-32.

1970. The Foraminifera of Sites 23-31, Leg 4. In Bader, R. G., Gerard, R. D., et al., Init. Repts. DSDP, 4: Washington (U.S. Govt. Printing Office), 577-643.

Bolli, H. M., and Bermudez, P. J., 1965. Zonation based on planktonic foraminifera of Middle Miocene to Pliocene warm-water sediments. Boll. Infor. Asoc. Venez. Geol., Min. Pet., 8:119-149.

Bolli, H. M., and Premoli-Silva, I., 1973. Oligocene to Recent planktonic foraminifera and stratigraphy of the Leg 15 sites in the Caribbean Sea. In Eger, N. T., and Saunders, J. B., et al., Init. Repts. DSDP, 15: Washington (U.S. Govt. Printing Office), 475-498.

Bolli, H. M., and Saunders, J. B., 1982. Globorotalia mayeri and its relationship to Globorotalia siakensis and Globorotalia continuosa. J. Foram. Res., 12:39-50.

1985. Oligocene to Holocene low-latitude planktic foraminifera. In Bolli, H. M., Saunders, J. B., and Perch-Nielsen, K. (Eds.), Plankton Stratigraphy: Cambridge (Cambridge Univ. Press), 155-262.

Cita, M. B., 1973. Pliocene biostratigraphy and chronostratigraphy. In Ryan, W.B.F., Hsü, K. J., et al., Init. Repts. DSDP, 13 (Pt. 2): Washington (U.S. Govt. Printing Office), 1343-1379.

Cordey, W. G., 1967. The development of Globigerinoides ruber (d'Orbigny, 1839) from the Miocene to Recent. Paleontology, 10:647-659.

Damuth, J. E., 1977. Late Quaternary sedimentation in the western equatorial Atlantic. Sedimentology, 26:825-834.

Droxler, A. W., and Schlager, W., 1985. Glacial versus interglacial sedimentation rates and turbidite frequency in the Bahamas. Geology, 13:799-802.

Droxler, A. W., Schlager, W., and Whallon, C. C., 1983. Quaternary aragonite cycles and oxygen isotope record in Bahamian carbonate ooze. Geology, 11:235-239.

Haq, B. U., Hardenbol, J., and Vail, P. R., 1987. Chronology of fluctuating sea levels since the Triassic ( 250 million years ago to Present). Science, 235:1156-1167.

Kennett, J. P., and Srinivasan, M. S., 1983. Neogene Planktonic Foraminifera: A Phylogenetic Atlas: Stroudsburg, PA (Hutchinson Ross Publ. Co.).

Kier, J. S., and Pilkey, O. H., 1971. The influence of sea level changes on sediment carbonate mineralogy, Tongue of the Ocean, Bahamas. Mar. Geol., 11:189-200.

Lamb, J. L., and Beard, J. H., 1972, Late Neogene planktonic foraminifers in the Caribbean, Gulf of Mexico, and Italian stratotypes. Univ. Kansas Paleontol. Contrib., Art. 57 (Protozoa 8), 1-67.

Lamb, J. L., and Stainforth, R. M., 1976. Unreliability of Globigerinoides datum. AAPG Bull., 60:1564-1569.

Lynts, G. W., Judd, J. B., and Stenham, C. F., 1973. Late Pleistocene history of Tongue of the Ocean, Bahamas. Geol. Soc. Am. Bull., 84: 2605-2684. 
Melillo, A. J., 1985. Late Oligocene to Pliocene sea-level cycle events in the Baltimore Canyon Trough and the western North Atlantic basin (Ph.D. dissert.). Rutgers University, New Brunswick, NJ.

Miller, K. G., Mountain, G. S., and Tucholke, B. E., 1985a. Oligocene glacio-eustasy and erosion on the margins of the North Atlantic. Geology, 13:10-13.

Miller, K. G., Aubry, M. P., Khan, M. J., Melillo, A. J., Kent, P. V., and Berggren, W. A., 1985b. Oligocene to Miocene bio-, magneto-, and isotopic stratigraphy of the western North Atlantic. Geology, 13: 257-261.

Miller, K. G., and Hart, M. B., 1987. Cenozoic planktonic foraminifers and hiatuses on the New Jersey slope and rise: Deep Sea Drilling Project Leg 95, Northwest Atlantic. In Poag, C. W., Watts, A. B., et al., Init. Repts. DSDP, 95: Washington (U.S. Govt. Printing Office), 253-265.

Miller, K. G., Melillo, A. J., Mountain, G. S., Farre, J. A., and Poag, C. W., 1987. Middle to late Miocene canyon cutting on the New Jersey continental slope: Biostratigraphic and seismic stratigraphic evidence. Geology, 15:509-512.

Moore, C. H., Graham, E. A., and Land, L. S., 1976, Sediment transport and dispersal across the deep fore-reef and island slope $(-55 \mathrm{~m}$ to $-305 \mathrm{~m}$ ), Discovery Bay, Jamaica. J. Sediment. Petrol., 46: 174-187.

Mullins, H. T., Neumann, A. C., Wilber, R. J., Hine, A. C., and Chinburg, S. J., 1980. Carbonate sediment drifts in northern Straits of Florida. AAPG Bull., 64:1701-1717.

Olsson, R. K., Melillo, A. J., and Schreiber, B. L., 1987. Miocene sea level events in the Maryland coastal plain and the offshore Baltimore Canyon Trough. In Ross, C. A., and Haman, D. (Eds.), Timing and Depositional History of Eustatic Sequences: Constraints on Seismic Stratigraphy: Cushman Found. Foram. Res.

Olsson, R. K., and Wise, S. W., Jr., 1987. Upper Paleocene to middle Eocene depositional sequences and hiatuses in the New Jersey Atlantic Margin. In Ross, C. A., and Haman, D. (Eds.), Timing and Depositional History of Eustatic Sequences: Constraints on Seismic Stratigraphy: Cushman Found. Foram. Res.

Poag, C. W., and Low, D., 1987. Unconformable sequence boundaries at Deep Sea Drilling Project Site 612, New Jersey Transect: their characteristics and stratigraphic significance. In Poag, C. W., Watts, A. B., et al., Init. Repts. DSDP, 95: Washington (U.S. Govt. Printing Office), 453-498.
Rögl, F., 1974. The evolution of the Globorotalia truncatulinoides and Globorotalia crassaformis group in the Pliocene and Pleistocene of the Timor Trough, DSDP Leg 27, Site 262. In Veevers, J. J., Heirtzler, J. R., et al., Init. Repts. DSDP, 27: Washington (U.S. Govt. Printing Office), 743-767.

Saito, T., Thompson, P. R., and Breger, D., 1981. Systematic Index of Recent and Pleistocene Planktonic Foraminifera: Tokyo (University of Tokyo Press).

Schlager, W., and Ginsburg, R. N., 1981. Bahama carbonate platformsthe deep and the past. Mar. Geol., 44:1-24.

Schlager, W., and Chermak, A., 1979. Sediment facies of platform-basin transition, Tongue of the Ocean, Bahamas. In Doyle, L. J., and Pilkey, O. H. (Eds.), Geology of Continental Slopes. Soc. Econ. Paleontol. Mineral. Spec. Publ., 27:193-208.

Shanmugam, G., and Moiola, R. J., 1982. Eustatic control of turbidites and winnowed turbidites. Geology, 10:231-235. 1983. Reply to Comment on "Eustatic control of turbidites and winnowed turbidites." Geology, 11:58-60. 1984. Eustatic control of calciclastic turbidites. Mar. Geol., $56: 273-278$

Stainforth, R. M., Lamb, J. L., Luterbacher, R., Beard, J. H., and Jeffords, R. M., 1975. Cenozoic Planktonic Foraminiferal Zonation and Characteristics of Index Forms. Univ. Kansas Paleontol. Contrib., Art. 62.

Thiede, J., 1981. Reworked neritic fossils in upper Mesozoic and Cenozoic central Pacific deep-sea sediments monitor sea-level changes. Science, 211:1422-1424.

Vail, P. R., Mitchum, R. M., Jr., Todd, R. G., Widmier, J. M., Thompson, S., III, Sangree, J. B., Bubb, J. N., and Hatlelid, W. G., 1977. Seismic stratigraphy and global changes of sea level. In Payton, C. E. (Ed.), Seismic Stratigraphy-Applications to Hydrocarbon Exploration. AAPG Mem., 26:49-212.

Vail, P. R., and Hardenbol, J., 1979. Sea-level changes during the Tertiary. Oceanus, 22:71-79.

Vail, P. R., Mitchum, R. M., Jr., Shipley, T. H., and Buttler, R. T., 1980. Unconformities of the North Atlantic. Phil. Trans. R. Soc. London, 294(A):137-155.

Date of initial receipt: 8 April 1987

Date of acceptance: 13 January 1988

Ms 101B-128 

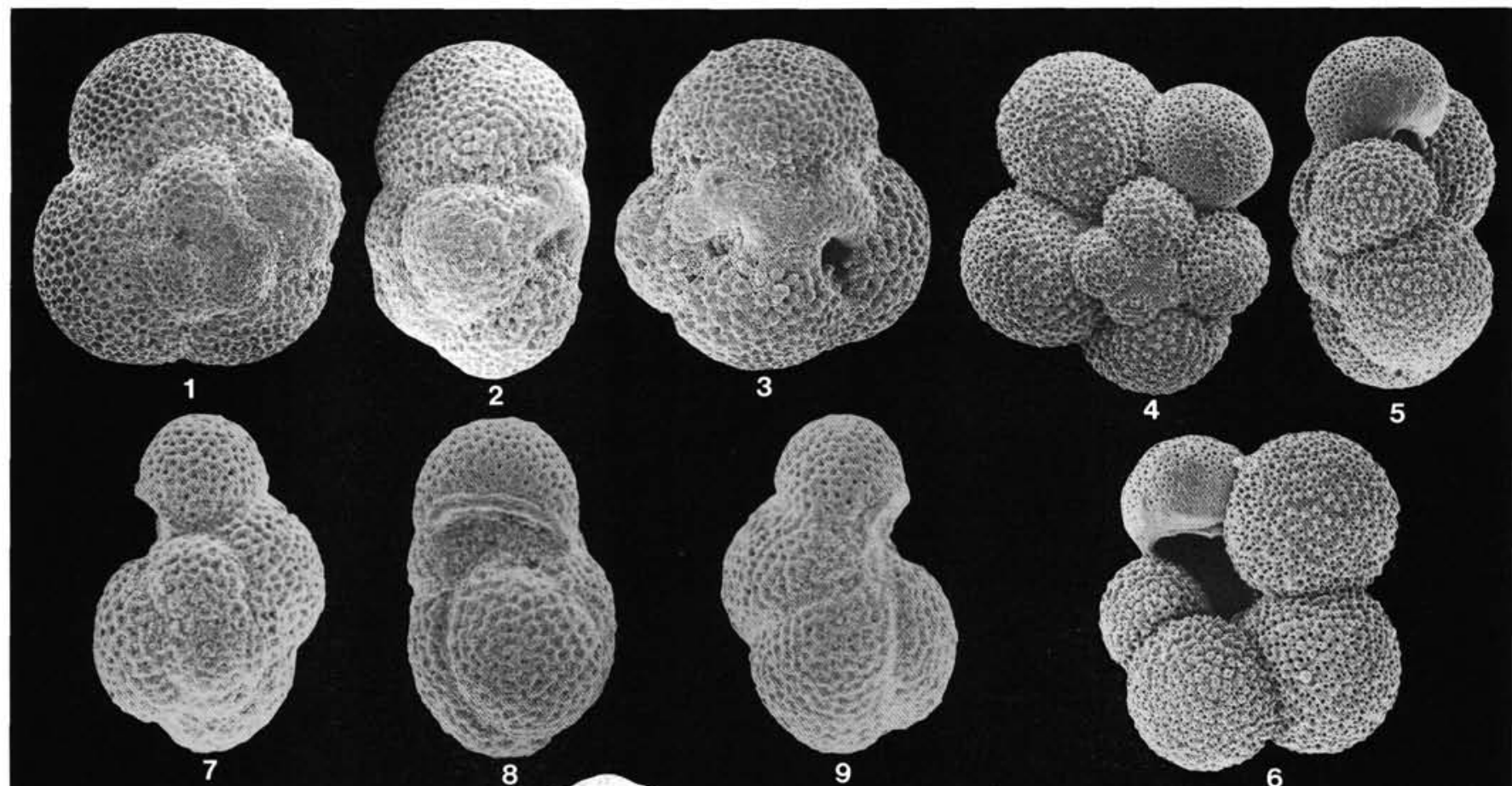

4

5

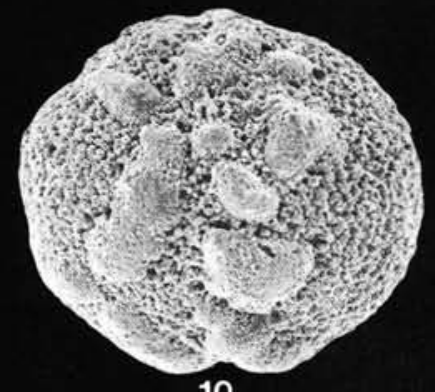

10

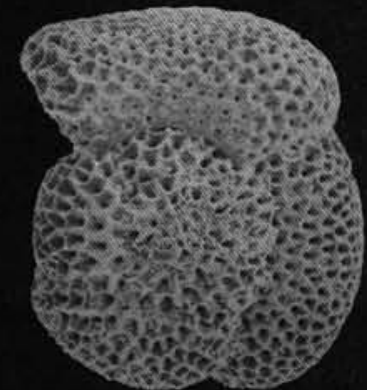

14

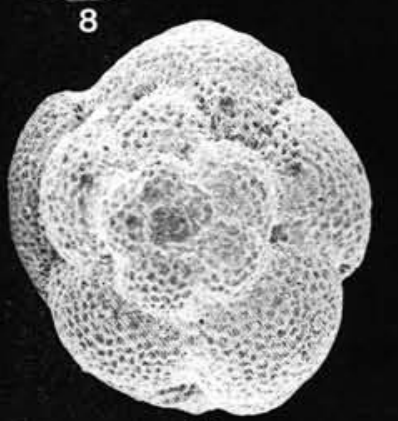

11

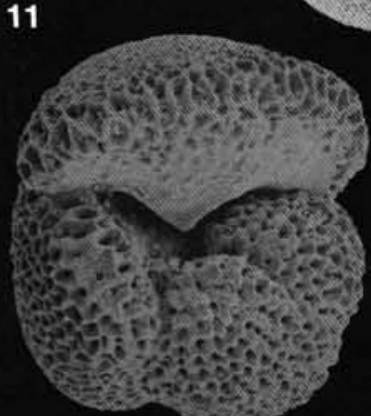

15

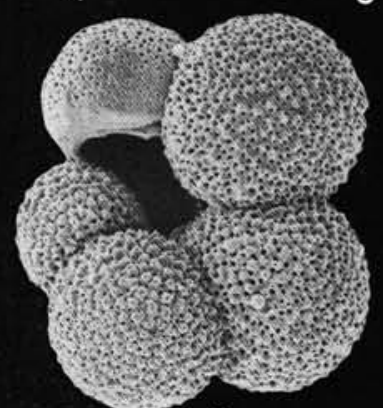

6

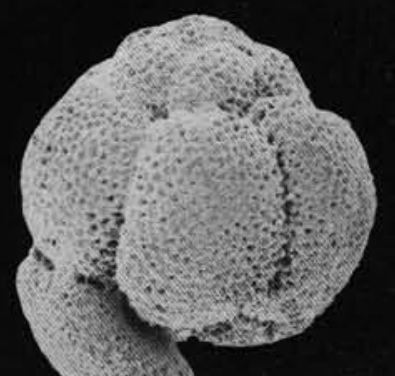

12

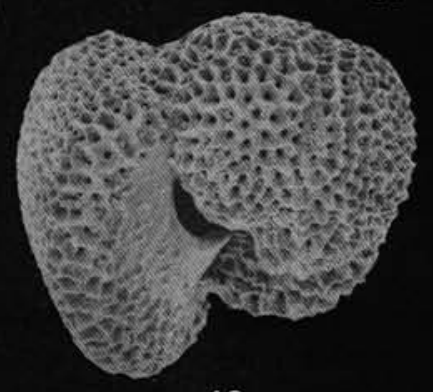

16

Plate 1. 1-3. Catapsydrax dissimilis (Cushman and Bermudez), Sample 101-627B-14H, CC, (1) spiral view, $\times 80 ;(2)$ edge view, $\times 75 ;(3)$ umbilical view, $\times 75$. 4-6. Globigerina calida calida (Parker), Sample 101-626C-5X-1, 25-27 cm, (4) spiral view; (5) apertural view; (6) umbilical view, all $\times 105$. 7-9. Globigerina nepenthes Todd, Sample 101-627B-10H-5, 25-27 cm; (7) spiral view; (8) umbilical view; (9) side view, all $\times 110$. 10. Globigerinatella insueta Cushman and Stainforth, Sample 101-627B-16H, CC, $\times 105$. 11-13. Globoquadrina altispira altispira (Cushman and Jarvis), Sample 101-628A-16H, CC, (11) spiral view; (12) side view; (13) umbilical view; $(11,12, \times 75 ; 13, \times 78)$. 14-16. Globoquadrina dehiscens (Chapman, Parr, and Collins), Sample 101-627B-12H-5, 25-27 cm, (14) spiral view; (15) umbilical view; (16) apertural view, all $\times 110$. 


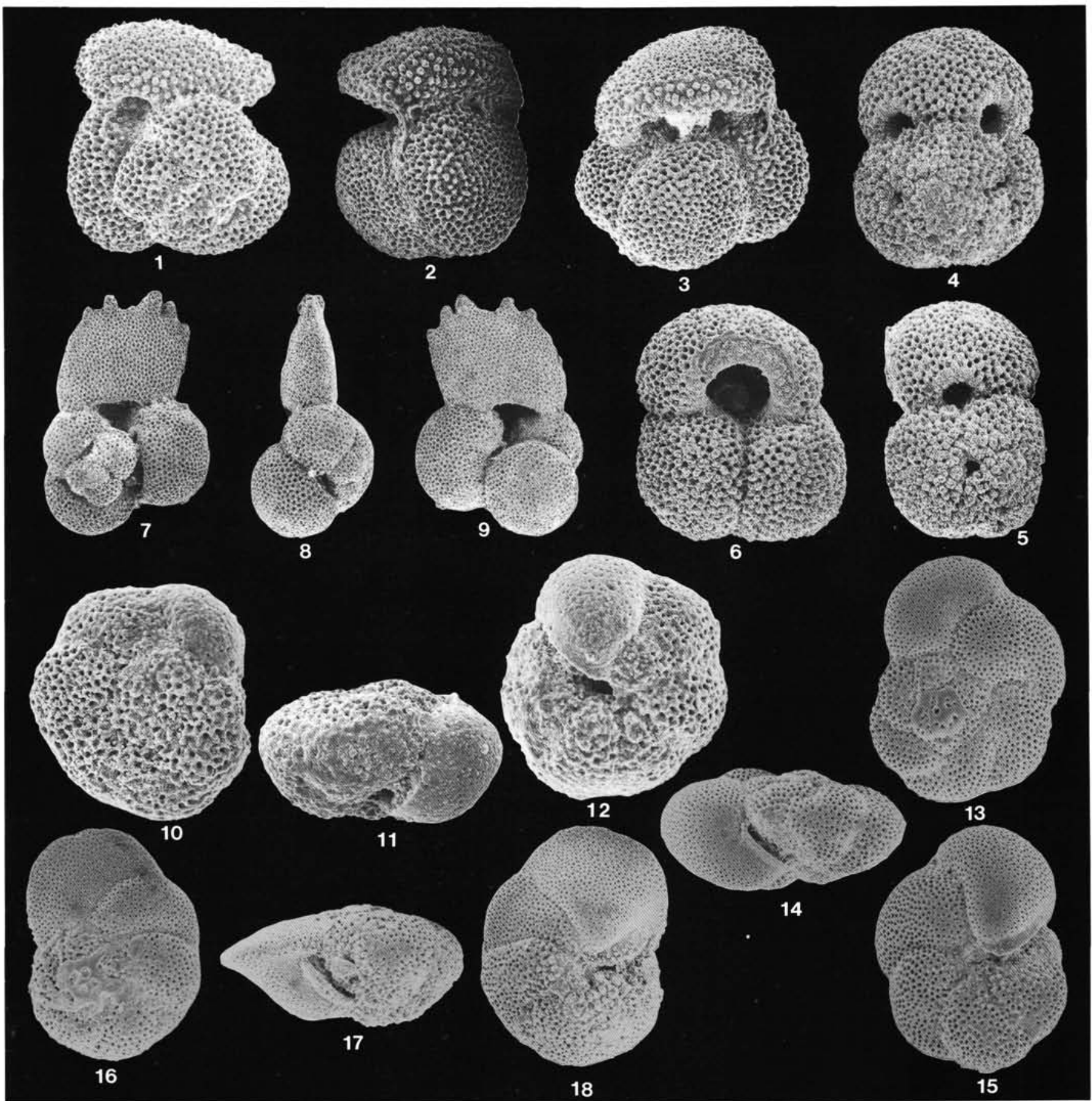

Plate 2. 1-3. Globigerinoides extremus Bolli, Sample 101-626C-9X-1, 21-23 cm, (1) spiral view; (2) side view; (3) umbilical view, all $\times 108$. 4-6. Globigerinoides subquadratus Bronnimann, Sample 101-627B-16H, CC, (4) spiral view; (5) side view; (6) umbilical view, all $\times 105$. 7-9. Globigerinoides trilobus fistulosus (Schubert), Sample 101-630A-4H-3, 25-27 cm, (7) spiral view, $\times 48$; $(8)$ side view, $\times 50$; (9) umbilical view, $\times 49$. 10-12. Globorotalia kugleri Bolli, Sample 101-627B-19X, CC, (10) spiral view, $\times 235$, (11) apertural view, $\times 215 ;(12)$ umbilical view, $\times 205$. 13-15. Globorotalia fohsi peripheroronda Blow and Banner, Sample 101-627B-15H, CC, (13) spiral view; (14) apertural view; (15) umbilical view, all $\times 110$. 16-18. Globorotalia fohsi peripheroacuta Blow and Banner, Sample 101-626B-5W-2, 25-27 cm, (16) spiral view; (17) apertural view; (18) umbilical view, all $\times 110$. 


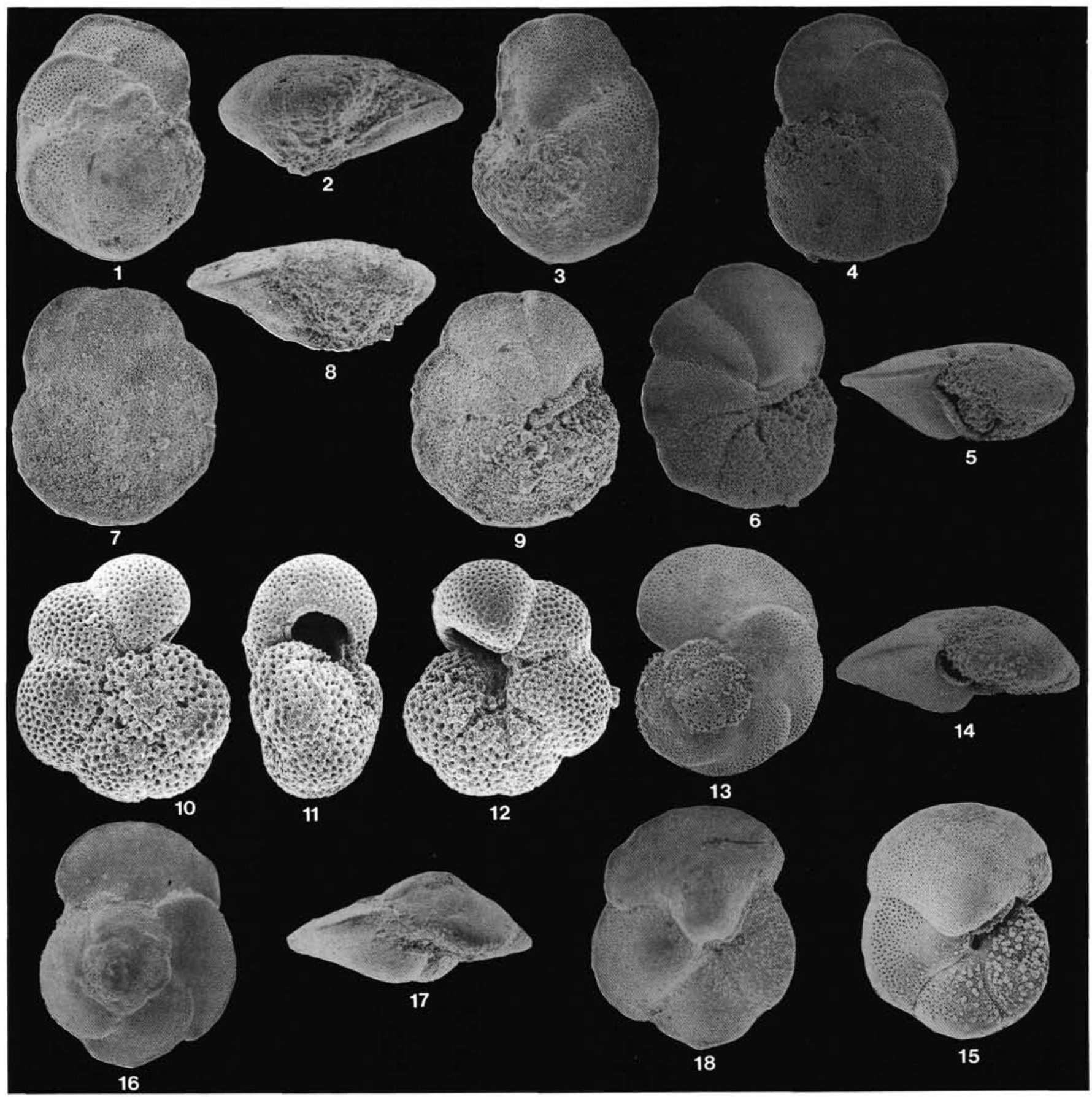

Plate 3. 1-3. Globorotalia fohsi fohsi Cushman and Ellisor, Sample 101-628A-15H-3, 25-27 cm, (1) spiral view; (2) apertural view; (3) umbilical view, all $\times 90$. 4-6. Globorotalia fohsi lobata Bermudez, Sample 101-628A-15H, CC, (4) spiral view, $\times 78 ;(5)$ apertural view, $\times 78 ;(6)$ umbilical view, $\times 76$. 7-9. Globorotalia fohsi robusta Bolli, Sample 101-628A-14H-3, $25-27 \mathrm{~cm}$, (7) spiral view, $\times 70 ;(8)$ apertural view, $\times 74 ;(9)$ umbilical view, $\times 72$. 10-12. Globorotalia mayeri Cushman and Ellisor, Sample 101-627B-12H, CC, (10) spiral view; (11) apertural view; (12) umbilical view, all $\times 115$. 13-15. Globorotalia margaritae margaritae Bolli and Bermudez, Sample 101-628A-8H, CC, (13) spiral view; (14) apertural view; (15) umbilical view, all $\times 90$. 16-18. Globorotalia margaritae evoluta Cita, Sample 101-630A-14H-3, 25-27 cm, (16) spiral view, $\times 66$; (17) apertural view, $\times 68$; (18) umbilical view, $\times 68$. 


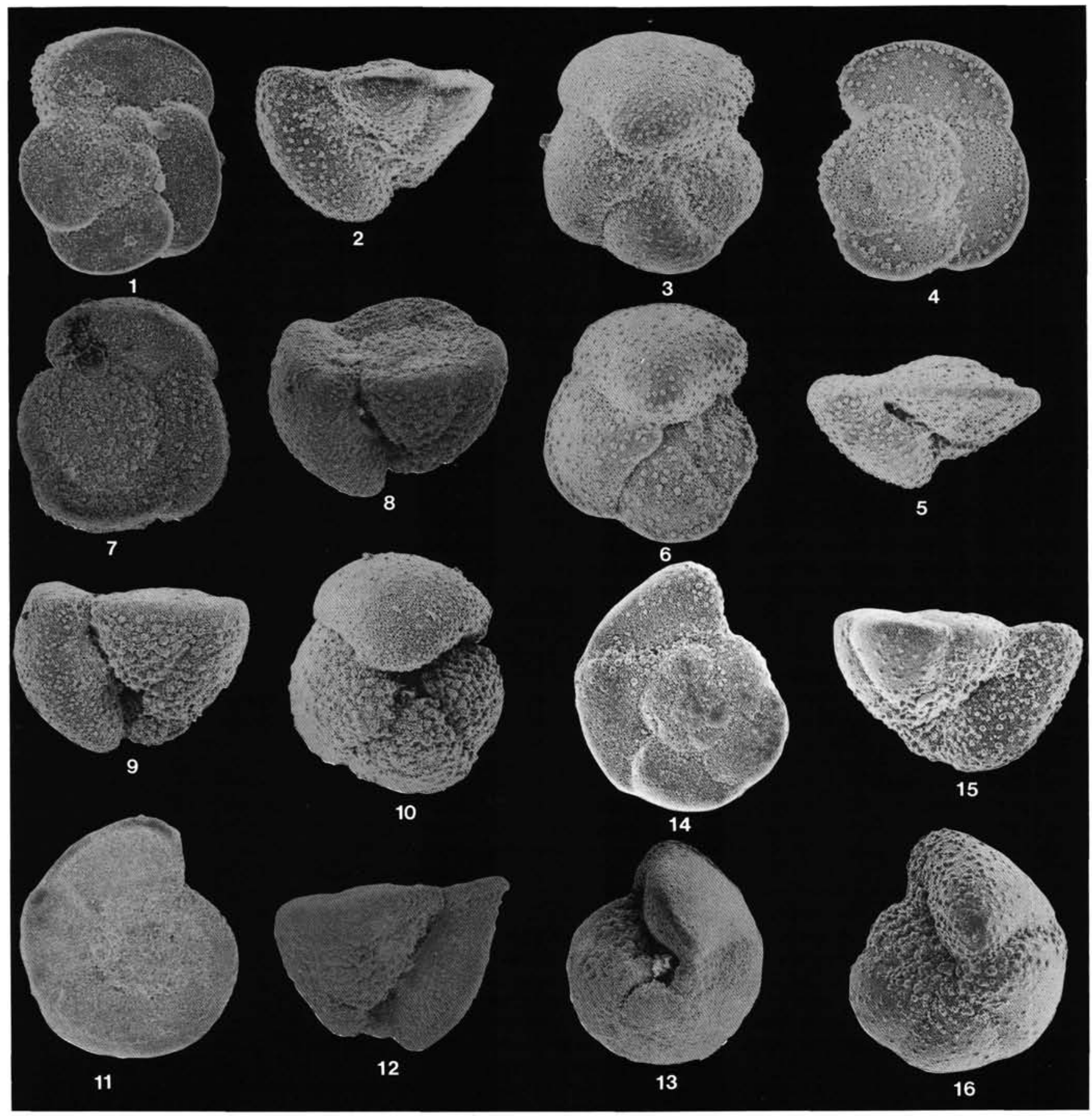

Plate 4. 1-3. Globorotalia crassaformis crassaformis (Galloway and Wissler), Sample 101-630-6H-4, $25-27 \mathrm{~cm}$, (1) spiral view; (2) apertural view; (3) umbilical view, all $\times 125$. 4-6. Globorotalia crassaformis viola Blow, Sample 101-633A-5H-3, 25-27 cm, (4) spiral view; (5) apertural view; (6) umbilical view, all $\times 100$. 7-10. Globorotalia crassaformis hessi Bolli and Premoli-Silva, Sample 101-632A-3H-3, 25-27 cm, (7) spiral view, $\times 95 ;(8)$ oblique view, $\times 90$; (9) apertural view, $\times 85$; (10) umbilical view, $\times 95$. 11-13. Globorotalia truncatulinoides truncatulinoides (d'Orbigny), Sample 101-632A-3H-3, 25-27 cm, (14) spiral view, $\times 66$; (15) apertural view, $\times 66$; (16) umbilical view, $\times 60$. 14-16. Globorotalia flexuosa $($ Koch), Sample $101-632 \mathrm{~A}-3 \mathrm{H}-2,25-27 \mathrm{~cm}$, (14) spiral view; (15) apertural view; (16) umbilical view, all $\times 42$. 


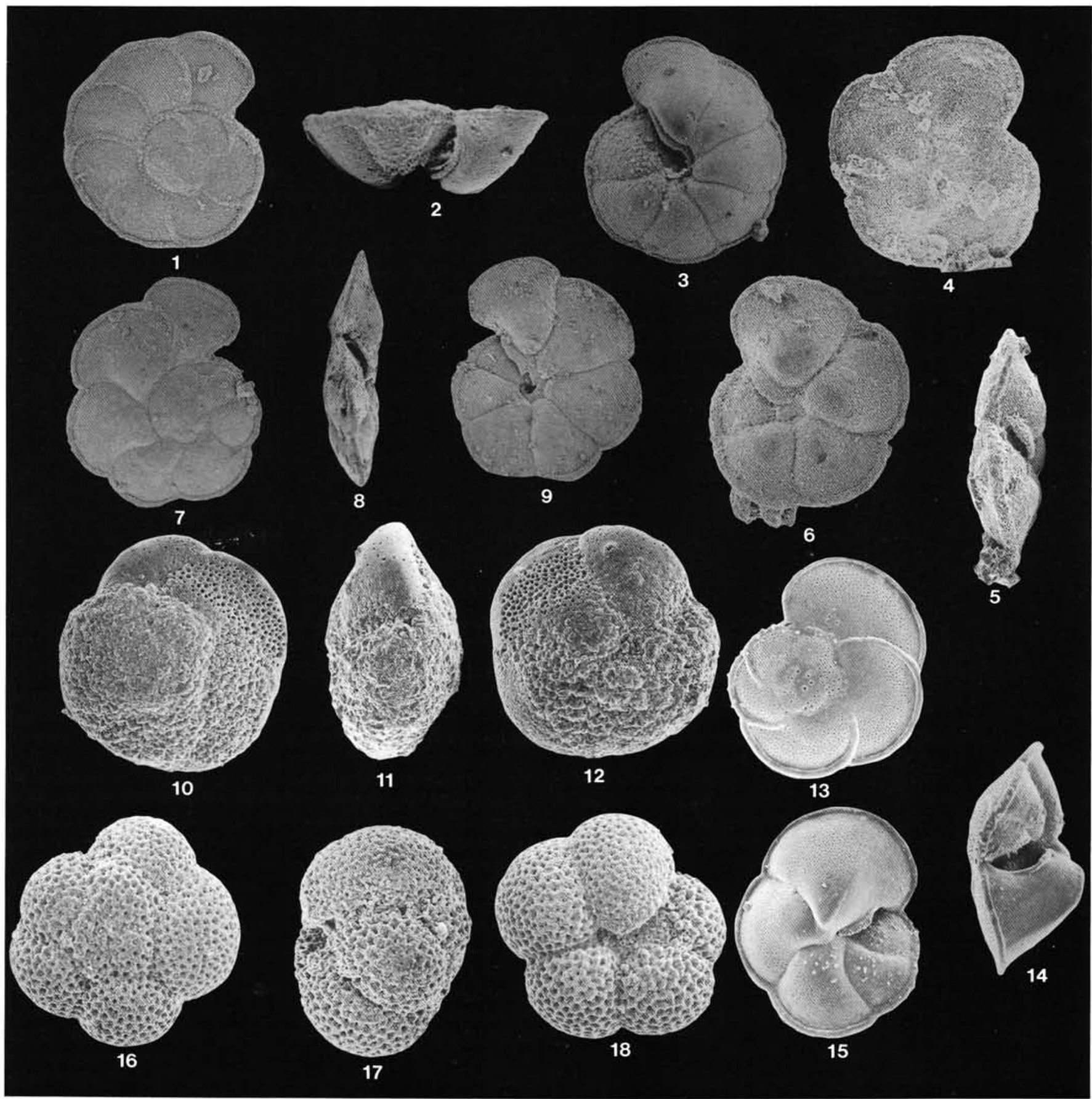

Plate 5. 1-3. Globorotalia miocenica Palmer, Sample 101-630A-2H-4, 25-27 cm, (1) spiral view; (2) apertural view; (3) umbilical view, all $\times 75$. 4-6. Globorotalia exilis Blow, Sample 101-630A-4H-3, 25-27 cm, (4) spiral view; (5) apertural view; (6) umbilical view, all $\times 75$. 7-9. Globorotalia pertenuis Beard, Sample 101-630A-4H-3, 25-27 cm, (7) spiral view; (8) apertural view; (9) umbilical view, all $\times 50$. 10-12. Globorotalia juanai Bermudez and Bolli, Sample 101-628A-9H, CC, (10) spiral view; (11) apertural view; (12) umbilical view, all $\times 80$. 13-15. Globorotalia ungulata Bermudez, Sample 101-627B-1H-4, 25-27 cm, (13) spiral view, $\times 92$; (14) apertural view, $\times 93 ;$ (15) umbilical view, $\times 92 ; 16-18$. Neogloboquadrina acostaensis (Blow), Sample 101-630A-13H-3, 25-27 cm, (16) spiral view, $\times 125$; (17) apertural view, $\times 125 ;(18)$ umbilical view, $\times 135$. 


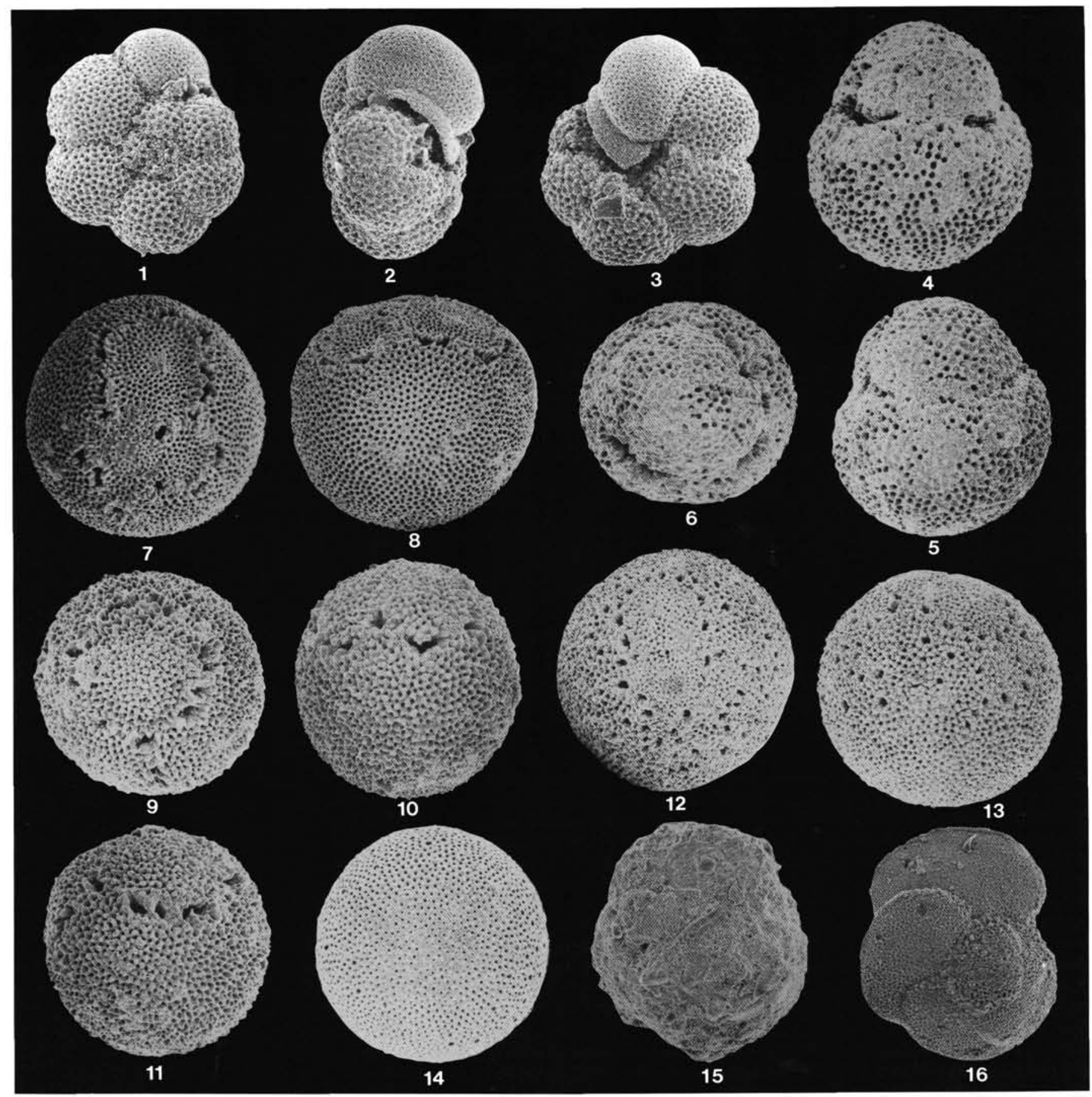

Plate 6. 1-3. Neogloboquadrina humerosa (Takayanagi and Saito), Sample 101-627B-7H, CC, (1) spiral view; (2) apertural view; (3) umbilical view, all $\times 160$. 4-6. Praeorbulina sicanus (DeStefani), Sample 101-627B-18X, CC, $(4,5)$ side view, $\times 100, \times 93 ;(6)$ spiral view, $\times 93$. 7-11. Praeorbulina glomerosa glomerosa (Blow) $(7,8)$ Sample 101-627B-16H, CC, (7) spiral view; (8) side view, both $\times 95$; $(9-11)$, Sample 101-627B-16H, CC, (9) spiral view, $\times 88,(10)$ side view, $\times 88$; (11) side view, $\times 90$. 12-13. Orbulina suturalis Bronnimann, Sample 101-628A-14H-3, 25-27 cm, (12) spiral view, $\times 100$; (13) side view, $\times 95$. 14. Orbulina universa d'Orbigny, Sample $101-633 \mathrm{~A}-4 \mathrm{H}-5,25-27 \mathrm{~cm}, \times 80$. 15-16. Specimen of Globorotalia margaritae s.1. in matrix, Sample 101-633A-5H-6, $25-27 \mathrm{~cm}$; (15) before ultrasonic cleaning, $\times 73$; (16) afterwards, $\times 83$. 